\title{
EFEITOS DA VIOLAÇÃO DA SUPOSIÇÃO DE INDEPENDENNCIA NA ANÁLISE DE VARIÂNCIA DO MODELO LINEAR DE GAUSS-MARKOV COM UM E DOIS FATORES
}

Tese apresentada à Escola Superior de Agricultura "Luiz de Queiroz", da Universidade de São Paulo, para obtenção do título de Doutor em Agronomia: Área de Concentração: Estatística e Experimentação Agronômica. 


\title{
EFEITOS DA VIOLAÇÃO DA SUPOSIC̣̃̃O DE INDEPENDÊNCIA NA ANALISE DE VARIÂNCIA DO MODELO LINEAR DE GAUSS-MARKOV COM UM E DOIS FATORES
}

\author{
LUIZ CARLOS BAIDA \\ Licenciado em Matemática
}

Orientador: Prof. Dr. Antonio Francisco Iemma

Tese apresentada à Escola Superior de Agricultura "Luiz de Queiroz". da Universidade de São Paulo. para obtenção do título de Doutor em Agronomía : Area de Concentração: Estatistica e Experimentação Agronómica. 


\section{EFEITOS DA VIOLAÇÃO DA SUPOSIC̣ÃO DE INDEPENDÊNCIA NA ANALISE DE VARIÂNCIA DO MODELO LINEAR DE GAUSS-MARKOV COM UM E DOIS FATORES}

Aprovada em 20.03 .1980

Comissão Julgadora:

Prof. Dr. Antonio Francisco Iemma

ESALQ USP

Prof. Dr. Humberto de Campos

ESALQ USP

Profa. Dra. Maria Cristina Stolf Nogueira

ESALQ USP

Prof. Dr. Ddelar Leite Linhares

Prof. Dr. João Riboldi

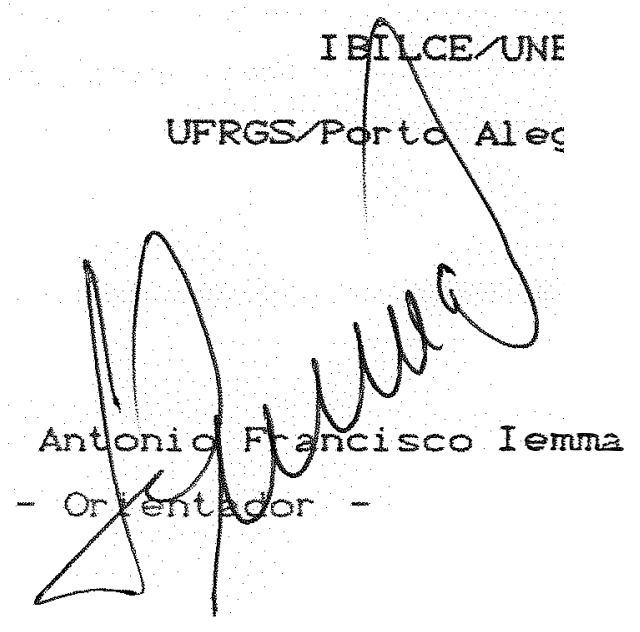


Aos meus pais e irmãos,

ofereco.

A mirha esposa Marcia. meus filhos:

Eduardo Augusio

Fernando Cesar

Karina Elisa.

dedico. 


\section{AGRADECIMENTOS}

Ao Dr. Antonio Francisco Iemma, Professor Associado do Departamento de Matematica e Estatistica da ESALQ - USP, pelas valiosas sugestöes e orientaça dest.e trabal ho.

Aos professores Dr. Antonio Francisco Iemma, Dr. Décio Barbin e Dr. Roberto Simionato Moraes, membros da Banca dos Exames Gerais de Qualificação, pela sugestão do assunto escolhido.

Á minha esposa Marcia pelo constante apoio. desde o inficio do Programa de Doutorado.

Aos proféssores do Curso de Pós-Graduação em Estatistica e Experimentação Agronómica da ESALQ - USP, na pessoa de seu coordenador, prof. Dr. Decio Barbin, pela receptividade e ensinamentos, em especial aos professores Dr. Humberto de Campos e Dr. Decio Barbin. pelo apoio e sincera amizade.

Ao professor Dr. Ruy Madsen Barbosa. Titular de Departamento de Análise Numerica e Estatistica do IBILCE - UNESP, pelos ensinamentos, incentivo e amizade.

Ao Departamento de Analise Numerica Estatistica do IBILCE - UNESP, na pessoa de seu chefe, profa. Dra. Sandra M. V. Ferreira Dias, pela oportunidade concedida para a realização deste Programa, em especial ao 
professor Dr. Antonio Espada Filho, pela atenção a nós dedicada.

Aos colegas do Curso de Pós-Graduação, pela amizade, leal dade e companheirismo.

Aos colegas da área de Estatística do Departamento de Análise Numérica e Estatistica do IBILCE UNESP, professores Antonio José Manzato e Fernando Ferrari, pelo apoio e em especial ao professor Dr. José Antonio Cordeiro, pela ajuda no desenvolvimento dos programas computacionais.

Ao Conselho Nacional de Desenvolvimento Cientifico e Tecnológico - CNPq, pela bolsa concedida.

Aos funcionarios do Departamento de Matemática e Estatistica da ESALQ - USP, pela amizade, cooperação e leal dade.

As secretarias do Departamento de Analise Numérica e Estatistica do IBILCE - UNESP. Il va, Nosmia e Marli, pela constante cooperação no decorer desse programa.

Aos técnicos do Laboratório de Computação do IBILCE - UNESP, Romil do e Getulio, pela cooperação.

‡ a Comissão Julgadora, pelas valiosas sugestżes, as quais vieram a enriquecer o trabalho. 
página

RESUMO $\quad \ldots \ldots \ldots \ldots \ldots \ldots \ldots \ldots \ldots \ldots \ldots \ldots \ldots \ldots \ldots \ldots \ldots \ldots \ldots \ldots \ldots$

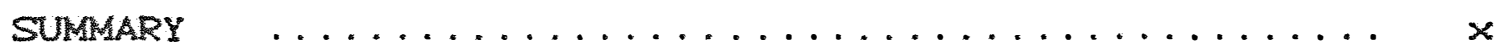

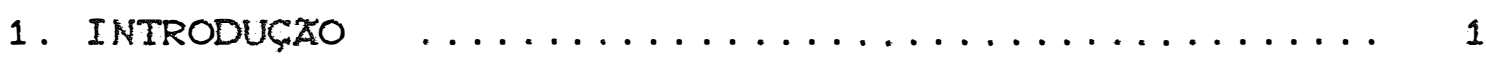

1.1. - O Problema $\ldots \ldots \ldots \ldots \ldots \ldots \ldots \ldots \ldots \ldots \ldots \ldots \ldots$

1.2. - Objetivos $\ldots \ldots \ldots \ldots \ldots \ldots \ldots \ldots \ldots \ldots \ldots \ldots \ldots \ldots \ldots$

2. REVISAO BIBLIOGRAFICA $\ldots \ldots \ldots \ldots \ldots \ldots$

2.1. - Consideraçes Iniciais $\ldots \ldots \ldots \ldots \ldots \ldots$

2.2. - Dois Artigos Básicos ............... 12

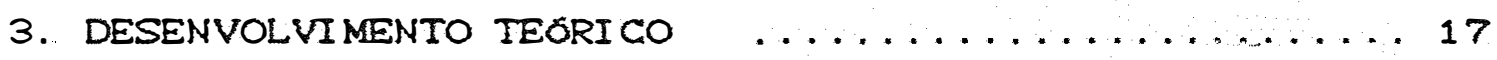

3.1. - Conceitos Introdutorios $\ldots \ldots \ldots \ldots \ldots \ldots \ldots$

3.2. - Modelos com Um Fator ..............21

3.2.1. - Um Fator Aleatorio ..........21

3.2.2. - Um Fator Fixo $\ldots \ldots \ldots \ldots \ldots$

3. 3. - Modelos com Dois Fatores Fixos e Balanceados. 47

3. 3.1. - Modelos sem interação...........47

3.3.2. - Modelos com interaçăo.......... 57

3. 3.3. - Procedimentos Alternativos ...... 69

3. 4. - Modelos Mistos com Dois Fatores .......... 83

3.4.1. - Modelos com interação...........83

3. 4.2. - Procedimentos Alternativos ......994 
4. RESULTADOS E DISCUSSATO $\ldots \ldots \ldots \ldots \ldots \ldots \ldots \ldots$

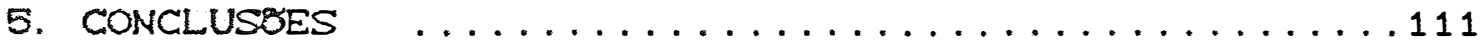

6. REFERENCI AS BI BLI CGRAFI CAS $\ldots \ldots \ldots \ldots \ldots \ldots \ldots$

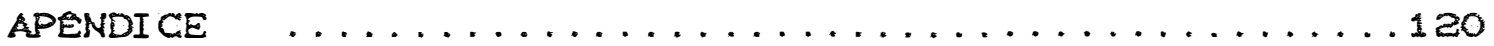


EFEITOS DA VIOLAÇÃO DA SUPOSIÇÕO DE INDEPENDÊNCIA NA ANALISE DE VARIÂNCIA DO MODELO LINEAR DE GAUSS-MARKOV COM UM E DOIS FATORES

\author{
Autor: LUIZ CARLOS BAIDA \\ Orientador: PROF. DR. ANTONIO FRANCI SCO IEMMA
}

RESUMO

Neste trabalho apresenta-se um estudo das implicações induzidas pela violação da pressuposição da independéncia sobre as probabilidades dos erros tipos I e II, inerentes ás hipóteses clássicas dos experimentos com um e dois fatores, analisados sob a estrutura padrao dos model os lineares de Gauss-Markov.

Para tal, tomam-se como ponto de partida os trabalhos de PERES (1985) e de SCARI ANO e DAVENPORT (1987), que mostram tais implicaçŏes em modelos com um rator rixo e balanceado, segundo um caso simples de equicorrelação, ao nivel nominal de significáncia 0,05 . Uma extensão desse trabalho para o caso não balanceado é feita segundo a equicorrelação já considerada. Estudam-se também tais implicaçós para modelos com um fator al eatório. 
Para modelos com dois fatores fixos e balanceados, estudam-se trés casos de equicorrelação. enquanto que para modelos mistos, dois casos são estudados. Tabelas das probabilidades dos erros tipos I e II para cada caso de equicorrelação e para cada configuração do modelo estudado, são construidas, tornando possivel avaliar o viés ao nivel nominal de significáncia 0,05. Dentre outras observaçzes, pode-se notar que a magnitude do erro cometido é diretamente proporcional à magnitude do coeficiente de correlação intraclasse, ao tamanho das amostras e ao número de niveis dos fatores envolvidos no planejamento.

Ademais, para cada caso de equicorrelaçăo e modelo estudado. procurou-se uma soluçăo alternativa que possibilitasse efetuar os testes das hipoteses principais. sem viés nas probabilidades dos erros tipos I e II, através de uma análise de variáncia univariada usual. Esse procedimento alternativo foi obtido atraves de uma modificação no planejamento, considerando-se "b" repetiçőes independentes entre si, acarretando a inclusăo do rator aleatório "repetição" no modelo. Para os casos em que tal procedimento apresentou resultados favoraveis, foram aqui relacionados, em caso contrário, não o foram.

Para o cálculo das probabilidades dos erros tipo II, utilizou-se a aproximação da distribuiçăo $F$ não central para a $F$ central, igualando seus tres primeiros momentos, conforme sugerido em IIKU C1985). O cklculo 
definitivo dessas probabilidades foi feito através da transformaçăo da $F$ central para a funçăo Beta incompleta, e do emprego das sub-rotinas envolvidas neste cálculo, dadas em Fortran e em Pascal por PRESS et alli (1986). 


\title{
THE EFFECTS OF VIOLATION OF INDEPENDENCE ASSUMPTION IN THE VARIANCE ANALYSIS OF GAUSS-MARKOV'S LINEAR MODEL WITH ONE AND TWO FACTORS
}

\author{
Author: LUIZ CARLOS BAIDA \\ Adviser: PROF. DR. ANTONIO FRANCISCO IEMMA
}

SUMMARY

This is a study of the implications induced by the violation of the independence assumptions on types I and II errors probabilities inherent to the classical hypotheses of the experiments with one and two factors. analyzed under the standard structure of Gauss-Markov's linear models.

For this purpose, PERES (1885) and SCARI ANO \& DAVENPORT (1987) were taken as starting points; they show these effects for models with one $f i x e d$ and bal anced factor according to a simple case of equicorrelation at the nominal level of significance 0,05 . An extension of this work to the unbalanced case is given according to the already considered equicorrelation. Such errects are also studied for models with one randon and bal anced factor.

For models with two fixed and balanced factors. up to three equicorrelation cases are studied. 
whereas for mixed models two cases are studied.

Tables of types I and II error probabilities. for each equicorrelation case and for each studied model. are given to show the bias of such probabilities at the 0,05 nominal significance level. Among other observations it can be noticed that the magnitude of the error is directly proportional to the magnitude of the intraclass correlation coefficient, to the size of the samples and to the number of levels invol ved at the design.

For each case of equicorrelation and model studied, an alternative solution was also sought so that tests could be performed for the main hypotheses, without any bias in the probabilities of types I and II errors through an usual analysis of univaried variance. This alternative procedure occurs through a modification of the design, considering " $b "$ as experimental replication independent among themselves, entailing the inclusion of the randon factor "replicas" in the model. Only the cases in which this procedure presented results were hereby 1 isted.

For the calculus of type II error probabilities the approximation of noncentral $F$ to central $F$ was used, equaliing their rirst three moments, as given by TIKU (1965).

The definitive calculus of these probabilities is done through the transformation of central $F$ into the 
xii.

incomplete Beta function and through the use of the sub-routines involved in this calculus as given in Fortran and Pascal by PRESS et alli (1086). 


\section{INTRODUCEO}

\subsection{O PROBLEMA}

Nos planejamentos experimentais que têm por objetivo verificar a existencia ou não de efeitos dos tratamentos envolvidos, é usual recorrer-se à análise de variancia, considerando válida todas as suposiçores básicas do modelo linear de Gauss-Markov.

Dentre essas suposiçớes, destaca-se a independéncia dos erros associados ao modelo em questão, assim como a sua normalidade. Assim, se o modelo em questão dado por ${ }_{n}^{Y_{1}}=n_{p_{1}}^{\theta_{1}}+n_{n_{1}}$ onde $Y$ é um vetor das observações, $X$ é a matriz do delineamento, $\theta$ é um vetor de parámetros $E$ um vetor de erros associado ao modelo, é usual considerar-se \& $\left.\cap N C O ; \sigma^{2} I\right)$, onde $\alpha^{2}$ é geralmente desconhecido (modelo ordinário de Gauss-Markov).

Dessa forma, os testes $F$ para as hipoteses principais são exatos quanto ao nível de significancia desejado, assim como seu poder segundo a hipótese alternativa assumida. 
Muitas vezes, no entanto, a matriz de variáncias e covariáncias associada ao erro não é da forma $\sigma^{2} I$, mas sim de uma forma mais geral $\Omega$, onde $\Omega$ é positiva definida.

Nossa principal preocupação se dá com as conseqüencias que podem acarretar, nos casos em que o experimentador considera seus dados segundo o modelo ordinário, quando na verdade seus erros não são independentes e sim correlacionados, segundo uma matriz $\Omega$ positiva definida. Nestes casos, quais seriam as verdadeiras probabilidades dos erros tipos I e II cometidos quando ele utiliza inadequadamente o modelo ordinário, visando um nivel de significancia pre-fixado a $? \quad \mathrm{Na}$ verdade, o nivel de significancia, neste caso, pode ser meramente nominal, pois as probabilidades dos erros podem afastar-se severamente daquel as pretendidas nos testes:

Muitos são os experimentos, nos mais diversos campos da pesquisa cientifica, nos quais essas correlaçäes podem estar presentes. Dentre outros, podemos citar aqueles cujas observaçŏes ocorrem: em grupos, em seqúencia e no espaço.

\subsection{OBJETIVOS}

A revisão bibliograrica eretuada revelou a existoncia de uma lacuna no tocante à quantificação eretiva 
das alteraçóes no nivel de significancia e no poder do teste, decorrentes da violação da hipótese de independéncia. Nesse contexto, temos como objetivo, através do uso de modelos lineares de efeitos fixos, aleatórios e mistos, efetuar um estudo teórico sobre o tema, bem como apresentar t.abelas que quantifiquem as variaçöes induzidas nas probabilidades de ocorrência dos erros dos tipos I e I.

Para tanto, será considerado o caso de correlação constante, caracterizado em várias configuraçäes, segundo o número de niveis dos fatores envolvidos e da magnitude da correlação considerada. 


\section{REVISÃO BIBLIOGRAF́ICA}

\subsection{CONSIDERACÕES INICIAIS}

A análise de variância, associada aos modelos lineares em geral, tem sido uma prática utilizada pelos pesquisadores das mais diversas áreas, a fim de comparar os efeitos produzidos pelos fatores dos modelos em estudo sobre suas unidades experimentais.

Não incomum presenciar-se, mesmo nos dias de hoje, o uso da análise de variância, sem que o pesquisador tenha garantias de que as suposições básicas para sua aplicabilidade estejam satisfeitas. Em geral consideram-se os dados como que oriundos de populações normais, independentes e de mesma variancia, isto é, supöe-se que os erros associados ao modelo tem distribuição de probabilidade normal com média $\mu=0$ e variancia $\alpha^{2}$, e são independentes entre si, acarretando uma matriz de variáncias e covariancias para o vetor de erros do tipo $\sigma^{2} I_{N}$, onde $I_{N}$ é uma matriz identidade de ordem $N$, $e N$ é o número total de observaçöes. 
$\mathrm{Na}$ verdade, se qualquer uma das suposiçరes citadas é violada, pode-se obter resultados desastrosos para os testes usuais quanto às hipoteses principais. Assim, vários autores, preocupados com esses fatos, colocam claramente em seus textos os efeitos dessas violaçäes, e procuram soluçōes $C$ muitas vezes aproximadas $>$ para superar esses problemas. BOX (1954-a) chama a atenção para - caso em que a matriz de variáncias e covariáncias é ainda diagonal, mas apresenta valores diferentes. Mostra que, nesse caso, boas aproximaçôs para o nivel de significancia podem ser obtidas utilizando-se aproximaçбes das distribuiçós de $x^{2} \cdot F$, para a análise de variáncia com um fator $f i x o$, se as variancias populacionais: são moderadamente diferentes.

BOX (1954-b), em trabal ho que é continuação do anterior, enfoca os efeitos das desigualdades das variancias e também das correlaçôs seriais de primeira ordem, para um planejamento cruzado com dois fatores, mostrando que mesmo nas suas soluçбes aproximadas, a correlação serial de primeira ordem dentro dos niveis de um dos fatores, afeta bastante o nivel de significancia para o teste da igualdade dos ereitos médios desse fator e pouco afeta o teste equivalente para o outro fator. Mostra ainda que, sob a hipóteses nula, direrenças nas variancias de coluna para coluna afetam sensivelmente o nivel de significancia para o teste da igualdade dos efeitos de colunas $\theta$ afeta em sentido contrário o teste para os 
efeitos das linhas. Para pequenas diferenças nas variáncias, nenhum efeito nos testes é considerado grande. De seus resultados apresentados, podemos ver ainda que correlaçzes positivas produzem efeitos mais desastrosos no nivel nominal de significáncia. SCHEFFE ( 1959 ), em seu clássico livro "The Analysis of Variance" "dedica o capitulo 10 para tratar dos efeitos das violações das suposiç̃es básicas, para uma análise de variancia. Ele estuda os efeitos das violações das suposiçães de normalidade dos erros, igualdade das variáncias dos erros e da independéncia estatística dos erros, para modelos com um fator fixo. Pode-se notar pela tabela 1.. descrita a seguir, que uma correlação serial de primeira ordem entre colunas afeta drasticamente o nivel de significancia para - teste dos efeitos de colunas, o que pode ser visto pela tabela 1. que mostra essas probabilidades segundo vários valores de $\rho$, visando um nivel de significáncia de 0.05 , num experimento cruzado 5x5. Neste caso, pode-se notar que o viés é maior para os testes de colunas.

Tabela 1. Niveis de significancia para os testes dos efeitos de linhas e colunas, num experimento cruzado 5x5, segundo os valores de $p$.

\begin{tabular}{c|rcccc}
$\rho$ & -0.4000 & -0.200 & 0.000 & 0.200 & 0.400 \\
\hline linhas & 0.0590 & 0.053 & 0.050 & 0.054 & 0.064 \\
colunas & 0.0003 & 0.010 & 0.050 & 0.130 & 0.250
\end{tabular}

Fonte: SCHEFFE (1959, p. 360$)$. 
Da literatura consultada, podemos ver que os problemas de correlaçőes nas observaçőes passam a ser uma preocupação de muitos autores. principalmente para os experimentos em que as medidas são repetidas sobre um mesmo individuo, como por exemplo DANFORD et alli (1860), que tratam do problema de medidas repetidas no tempo atraves das análises uni e multivariadas. Dado que os autores analisam dados multinormais que satisfazem a certas condiçơes de simetria $C$ equicorrelação $)$, as analises univariadas puderam ser aplicadas com exito e com muita facilidade. No entanto. mostram que se estas condições de simetria não estão satisfeitas. deve-se lançar mão da análise multivariada. Neste mesmo artigo, os autores testam a igualdade das variancias e covariancias, sob normalidade. atraves do teste proposto por WILKS (1846). usando 0 critério da razão de verossimilhança de Neyman-Pearson. posteriormente modificado por BOX C 1849). Então. se a hipotese de equicorrelação é rejeitada, utiliza-se a analise multivariada.

Ainda em se tratando de medidas repetidas. podemos citar GILL e HAFS ( 1871 ), que enfocam bem o caso de uma equicorrelação com medidas repetidas com animais. tratando de um exemplo em que o pesquisador analisa seus dados de duas formas diferentes, primeiro como um experimento completamente casualizado, onde não se faz presente a correlação, e. em seguida, como um Split-plot com matriz uniforme de variancias e covariancias. Enfocam as 
diferenças ocorridas nestes casos para os testes das hipoteses principais. O exemplo tratado enfoca um experimento com dois grupos de seis ratas cada um, onde num dos grupos as ratas estavam grávidas, e no outro não estavam. Para cada animal. foram feitas 4 observaçőes em 4 periodos de 4 dias cada um. O pêso em gramas de leite de cada período observado de cada animal foi registrado. Para cada grupo de 6 ratas. foi calculada a matriz de dispersao dos dados, com o objetivo de testar a uniformidade dessa matriz. Utilizaram o teste F aproximado, dado por Box(1950). para o teste da uniformidade.

HUYNH e FELDT (1970) preocuparam-se com as condiçros sob as quais as razöes dos quadrados medios tem distribuiçăes $F$ exatas, as quais dependem da matriz de variáncias e covariancias adotada para o modelo.

IEMSA (1981-1983) analisa o comportamento dos experimentos em parcelas subdivididas com tratamentos principais dispostos em blocos incompletos balanceados sob a estrutura uniforme de erros, na qual a correlação constante estava presente entre subparcelas de uma mesma parcela. Para assegurar a validade das pressuposiçŏes da estrutura de erros. o autor adota os testes de uniformidade e de homogeneidade das matrizes de variáncias e covariáncias envolvidas.

PRAET2 (1881) mostra os efeitos de autocorrelạ̧ão na analise de regressão múltipla. no que diz respeito ao viés provocado pelas correlaçôes nas 
esperanças das estatisticas $F \in R^{2}$ onde

$F=\frac{\hat{\beta} \cdot X(n-k)}{Z^{\prime} Z(k-1)}$ e $R^{2}=\frac{\beta X^{\prime} y}{Y^{\prime} y}$ para a regressão múltipla $n_{1}=x_{k} \hat{\beta}_{1}+n_{1}^{u_{1}}$, onde $Z^{\prime} Z=u^{\prime}\left(I-X\left(X^{\prime} X\right) X^{\prime}\right) u$. O autor mostra - viés provocado na $E(F)$ e $E\left(R^{2}\right)$ para $n=20,40$ e 100 , com coeficiente de correlaçăo $\rho=0.8 ; 0.5 ; 0.2 ; 0.0$; $-0.2 ;-0.5 ;-0.8$.

ANDERSEN et alli (1981) tratam da análise de variancia com dois fatores (linhas e colunas) na presença de erros correlacionados. Eles propoem aproximaçơes para as distribuiçōes dos testes usuais dos efeitos de linhas e de colunas. Essas aproximaçōes são estudadas com o auxilio de simulaçőes para modelos auto-regressivos de primeira ordem e modelos de media móvel de primeira ordem. Mostram também que, se não considerarmos tais correlaçōes, os resultados conclusivos podem ser desastrosos, principalmente quando se trata de medidas ao longo do tempo.

GILL e SHUKLA (1985-b) preocuparam-se com observações correlacionadas e mostraram que alguns planejamentos são ótimos quanto à sua eficiéncia quando se usa as estimativas de minimos quadrados generalizados para modelos auto-regressivos e de média móvel, cujas matrizes de variâncias e covariâncias são respectivamente dadas por:

$$
\begin{array}{ll}
\Omega=\sigma_{e}^{2}\left(1-\rho^{2}\right)^{-1} I_{b} \otimes H_{k}+\sigma_{\eta}^{2} I & \\
\Omega=\sigma_{b}^{2} I_{b} \otimes\left(I_{k}+\rho H_{k}\right)+\sigma_{\eta}^{2} I, & \text { onde }
\end{array}
$$


b é o número de blocos de tamanho $k ; H_{k}$ é uma matriz $k x k$, com as entradas $(i, j)$ dadas por $\rho^{|i-j|}, \operatorname{com}|\rho|<1 ; \quad N_{k}$ é uma matriz $k \times k$ com as entradas $(i, i+1)$ e $(i+1, i)$ iguais a "1" e "O" para as demais entradas.

Para essas estruturas de covariáncias, os autores mostraram, para blocos de 5 e 10 tratamentos, que usar mínimos quadrados generalizados para experimentos em blocos quase balanceados pode ser melhor que usar minimos quadrados ordinarios.

IEMMA (1985) desenvolve a teoria dos modelos lineares para experimentos com medidas repetidas através das matrizes de Helmert, mostrando que a utilização das matrizes de Helmert facilita as aplicaçóes dos modelos em tópicos como: obtenção da matriz amostral de variáncias e covariancias, testes para sua uniformidade, decomposição espectral, estimaç̋̃es por ponto, por intervalo e por região de funç̧̃es dos parámetros, estudo de formas quadráticas, testes de interesses, e assim por diante.

Como exemplo, enfoca o modelo dado por $y_{1 j}=\mu+v_{i}+\tau_{j}+e_{i j}$, onde $v_{i} e \tau_{j}$ representam os efeitos de individuos e repetição, respectivamente para $i=1,2, \ldots . p$ e $j=1,2, . . k$, cuja matriz de variancias e covariancias dos erros tem a seguinte estrutura

$$
\operatorname{cov}\left(e_{i j}, e_{i}, j\right)=\left\{\begin{array}{ccccc}
\sigma^{2} & \text { se } & i=i, & e & j=j \\
0 \sigma^{2} & \text { se } & i=i, & e & j \neq j \\
0 & \text { se } & i \neq i
\end{array}\right.
$$

para $-\frac{1}{k}<p<1$. Mostra também que a analise de 
variancia usual não apropriada para o teste de efeitos entre individuos.

$$
\text { REILMAN et alli (1985) mostraram que os }
$$

estimadores de máxima verossimilhança são preferiveis a outros estimadores, quando as observaçశes são equicorrelacionadas e a amostra suficientemente grande para modelos de regressão do tipo $Y=\alpha+\beta X$, onde as duas variáveis $X$ e $Y$ são medidas com erros, isto é $y_{i}=Y_{i}+v_{i}$ $\ominus x_{i}=x_{i}+u_{i}(i=1,2, \ldots, n) \quad \operatorname{com} \quad x \cap N\left(\mu_{x} ; \alpha_{x}^{2}\right)$, $v \cap N\left(O ; \sigma_{v}^{2}\right), u \cap N\left(O ; \sigma_{u}^{2}\right) e \operatorname{corr}\left(u_{i}, v_{j}\right)=\rho$ se $i=j$ e 0 se $i \neq j$.

HENDERSON ( 1986 ) tratou do problema que ele chama de " change-point ", que nada mais b́ do que seccionar uma sequência de $n$ variáveis aleatórias de modo que as primeiras $k<1 \leq k \leq n$ ) observaçöes venham de uma população e as demais observações de outra população, considerando a presença de correlação entre elas pois, segundo HENDERSON (1986), muitos autores tratam desse problema supondo independência entre as variáveis.

KENNY e JUDD $(1986)$ mostram o viés nas esperanças dos quadrados médios de tratamento e para o resíduo, quando a correl ação das observações se dá devido a grupos de observações, devido a observações sequenciais Cmodelos de média móvel e auto-regressivos l e devido ao espaço, para modelos com um fator e cuja variável observada é discreta. Fornecem também para cada caso uma forma de estimar as correlaçơes, mas não mostraram esses ereitos na 
forma das probabilidades dos erros.

ZIMMERMANN C 1987 ) estudou os efeitos da heterogeneidade das variáncias nos planejamentos em blocos ao acaso e quadrados latinos, segundo várias distribuições para o modelo, utilizando o teste $F$ para todas essas distribuições. Seus dados foram gerados segundo essas várias distribuições. Não considerou observações correlacionadas, mas apenas a heterogeneidade das variáncias. O autor concluiu, segundo seu experimento, que a não normalidade dos dados não afetou significamente o poder do teste F, fato que não ocorreu com a heterogeneidade que afetou o poder do teste F, principalmente quando a distribuição utilizada foi a normal.

\subsection{DOIS ARTIGOS BÁsicos}

Como acabamos de ver, muitos são os autores que se preocuparam com as correlações entre dados observados e com a heterogeneidade das variancias populacionais, abordando o fato das mais diversas formas teóricas e práticas.

Este trabalho também uma forma de registrar nossa preocupação com esses fatos e, nesse contexto, dois artigos foram básicos para nosso estudo, são eles: o artigo de SCARI ANO e DAVENPORT (1987) e o artigo de PERES ( 1985 ). 
linear com um fator fixo e balanceado $y_{i j}=\mu+\alpha_{i}+e_{i j}$ para $i=1,2, \ldots, m \quad e \quad j=1,2, \ldots, n$, cuja estrutura de erros $\&$ dada por

$$
\operatorname{cov}\left(e_{i j}, e_{i}, j,\right)=\left\{\begin{array}{lll}
\sigma^{2} & \text { se } i=i, & e j=j \\
\rho \sigma^{2} & \text { se } i=i, & e j \neq j
\end{array}\right.
$$

para $\frac{-1}{n-1}<\rho<1$, onde $\rho$ é coeficiente de correlação intraclasse.

A matriz de variancias e covariancias desse modelo é então dada por $\Omega=\sigma^{2} V$ onde $V$ é tal que $V \in \mathcal{Z}=\left\{V: V=c_{1} P_{1}+c_{2} P_{2}+c_{3} P_{3}\right.$, com $\left.c_{i}>0\right\}$

e $P_{i}$ é tal que $Y \cdot P_{i} Y$ são formas quadráticas que nos fornecem as somas de quadrados para a ANOVA, com $\left.P_{i} \cdot C i=1,2,3\right)$ matrizes projetores ortogonais $C P_{i} P_{j}=0$ para $i \neq j$, simétricas e idempotentes. Se $P_{1}+P_{2}+P_{3}=I_{N}$, ou seja, se $c_{1}=c_{2}=c_{3}=1$, então $V=I_{N}$, onde $N=m n$ - número total de observaçâes.

$$
\text { A hipótese de interesse é } H_{0}: \alpha_{i}=0 \text { vs } H_{i}: \alpha_{i} \neq 0
$$

para algum $i=1,2, \ldots, \mathrm{m}$.

Sob normalidade dos erros, isto é, o vetor de erros tendo distribuição multinormal $N\left(\theta, \alpha^{2} V\right)$, pela própria construção de $\mathfrak{\text { tem-se }}$

$$
\begin{gathered}
\left(Y P_{i} Y-c_{i} \alpha^{2}\right) \cap \chi^{2}\left(r\left(P_{i}\right), \lambda_{i}\right), \quad \text { onde } \\
\lambda_{i}=E\left(Y^{\prime}\right) P_{i} E(Y)-2 c_{i} \sigma^{2}, \quad \text { para } i=1,2,3 .
\end{gathered}
$$

- autor define a familia de variáveis aleatórias:

$F_{i j}\left(c_{i}, c_{j}\right)=\frac{c_{j}}{c_{i}} \frac{Y^{\prime} P_{i} Y / r\left(P_{i}\right)}{Y, P, Y / r\left(P_{j}\right)} \cap F\left[r\left(P_{i}\right), r\left(P_{j}\right), \lambda_{i}, \lambda_{j}\right]$ 
para $i \neq j, i, j=1,2,3$.

$$
\begin{aligned}
& \text { Fazendo-se } \quad S\left(R(\mu)=Y \cdot P_{\mathbf{a}} Y, \quad S Q(\alpha)=Y \cdot P_{\mathbf{2}} Y,\right. \\
& \text { SQResiduo }=Y^{\prime} P_{2} Y=Y^{\prime}\left(I_{N}-P_{1}-P_{3}\right) Y \text { e SOTotal }=Y^{\prime} I_{N} Y \text {, } \\
& \text { tem-se que } c_{1}=c_{2}=1 \Longrightarrow F_{12}(1,1)=F \text { é a estatistica } \\
& \text { "usual" para testar-se } H_{0} \text { vo } H_{1} \text {. No entanto, para que isso } \\
& \text { ocorra é necessário que } \mathrm{V}=I_{\mathrm{N}} \text {. }
\end{aligned}
$$

Então, sob a presença de equicorrelação,os testes usuais apresentam um viés nas probabilidades dos erros tipos I e II.

Se a correlação $\rho$ é tal que $\rho>$, tem-se $c_{1}=c_{3}=1+(n-1) \rho$ e $c_{2}=1-p$, acarretando que a P(errotipoI) $\left.=P\left(F_{12} C_{1}, c_{2}\right) \geq \frac{1-\rho}{1+(n-1) \rho} F_{\alpha}(m-1, N-1)\right) \geq \alpha$ e então os autores mostram essas probabilidades para vários valores de $n, m e \rho$ ao nível nominal de significancia $\alpha=0.05$. Mostram, ainda, a rápida convergéncia dessas probabilidades para "1" e tanto mais rápida quanto maiores os valores de $n, m e p$. Eles mostram também as probilidades dos erros tipo II para vários valores de $n, m, \rho e$ $\phi=\frac{2 c_{1} \lambda_{1}}{n}$, cujas probalidades decrescem para 0 valor zero rapidamente. Desenvolvimento análogo é feito para $\frac{-1}{n-1}<\rho<0$, as conclusaes se dão no caminho inverso àquelas obtidas para po,isto é, as probabilidades dos erros tipo I decrescem rapidamente para o valor zero, enquanto as probabilidades dos erros tipo II crescem para o valor 1. 
PERES (1885), utilizou o mesmo modelo com a mesma equicorrelação mostra os efeitos que essa equicorrelaçăo produz nas probabilidades dos erros tipo I, quando a análise de variáncia usual e aplicada. O autor não apresenta a classe $¥$ de matrizes de variáncias e covariancias e nem as probabilidades dos erros tipo II, como fazem os autores SCARIANO \& DAVENPORT (1887). Em contrapartida o autor apresenta como sugestão uma solução alternativa do problema, que consiste numa modificação do planejamento e consequentemente no modelo de análise, com base em repetiçбes independentes do experimento, a fim de assegurar o nivel de significáncia préfixado para o testo

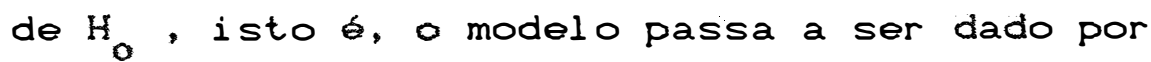

$$
\begin{aligned}
& y_{i j k}=\mu+\alpha_{i}+R_{j(i\}}+e_{i j k} \text { onde } i=1,2, \ldots, m \text { e } \\
& j=1,2, \ldots, b \text { e } k=1,2, \ldots, n, \text { sendo }{ }_{j(i)} \text { o efeito }
\end{aligned}
$$
de repetição dentro do nivel $i$ do fator $\alpha$, com as suposiçбes de que ${ }_{j(i)} \bullet e_{i j k}$ são variáveis aleatórias independentes com distribuiçбes multinormais de médias nulas e matrizes de variáncias e covariáncias dadas por $\sigma_{R}^{2} I_{N} \otimes I_{r b} \otimes U_{n} \operatorname{com} U_{n}=\sigma^{2}\left[(1-\rho) I_{n}+\rho E_{n}\right]$, respectivamente, onde $N=r b n \cdot I_{P} \Leftrightarrow$ a matriz identidade $e E_{P}$ a matriz de elementos unitários, de ordens P. Assim, a matriz de variáncias e covariáncias do modelo passa a ser dada por $\Omega=I_{r b} \otimes\left[\sigma_{R}^{2} E_{n}+U_{n}\right]$ e procedendo-se à análise de variáncia usual, considerando-se a restrição $\sum_{i=1}^{r} a_{i}=0$, - autor verifica que o teste para $H_{0}$ vo $H_{1}$ pode agora ser feito atraves do teste $F$, contra a soma de quadrados médio, 
devido a repetiçăo, sem que haja alteraçoses nas probabilidades do erro tipo I. 


\title{
3. DESENVOLVIMENTO TEÓRICO
}

\subsection{CONCEITOS INTRODUTORIOS}

\begin{abstract}
Apresenta-se aqui algumas consideraçăes teóricas que serão utilizadas por todo o trabalho. Sejam as matrizes de equicorrel ação $\Omega$ tais que:

$\Omega \in \mho=\left\{\Omega: \Omega=c_{0} P_{0}+c_{1} P_{1}+\ldots+c_{t} P_{t}\right.$, com $\left.c_{i}>0 P^{\prime} i=1,2, \ldots, t\right\}$ onde $Y$ 'P $P_{i} Y$ a $a$ soma de quadrados referente ao i-ésimo parámetro de interesse com $P_{i} P_{j}=0$ se $i \neq j$, $P_{i} P_{i}=P_{i}$ e $P_{i}=P_{i}$, para $i, j=1,2, \ldots, t$.

Seja também o teorema citado em SEARLE c1871p. 605 apresentado a seguir:

Teorema 1. Seja $x$ um vetor $n \times 1$ multinormal, $x \cap N(\tilde{\mu}, V)$, onde $\tilde{\mu}$ é o vetor de médias e $V$ é a matriz de variancias e covariancias da distribuição multinormal, $\theta \operatorname{sejam~} A_{i}$, $i=1,2, \ldots, p$ matrizes simétricas $n \times n$, de rank $k_{i}$, tais que $A=\sum_{i=1}^{p} A_{i}$, com $A$ simétrica de rank $k$. Então as formas quadráticas X'A $X$ têm distribuição $\chi^{2}$ com $k_{i}$ graus de liberdade e parámetro de não centralidade $\delta_{i}=\frac{1}{2} \tilde{\mu}^{\prime} A_{i} \tilde{\mu}$,
\end{abstract}


isto $\Leftrightarrow X \cdot A_{i} X \cap \chi^{2}\left(k_{i} ; \frac{1}{2} \tilde{\mu} \cdot A_{i} \tilde{\mu}\right)$, são duas a duas independentes X'A $X$ tem distribuição de $x^{2}$ com $k$ graus de liberdade e parámetro de não centralidade $\delta=\frac{\varepsilon}{2} \tilde{\mu}^{\prime} A \tilde{\mu}$. isto $e x \cdot A \times \cap x^{2}\left(k ; \frac{1}{2} \tilde{\mu} \cdot A \tilde{\mu}\right)$, se e somente se:

(i) quaisquer duas alternativas (a), (b) e (c) săo verdadeiras:

$$
\begin{aligned}
& \text { (a) } A_{i} V \text { é idempotente para } i=1,2, \ldots \text {. } \\
& \text { (b) } A_{i} V A_{j}=\emptyset \text { para todo } i<j \\
& \text { (c) } A V \text { idempotente }
\end{aligned}
$$$$
\text { ou }
$$

(ii) (c) é verdade e (d) $k=\sum_{i=1}^{p} k_{i}$ ou

(ili) $(c)$ é verdade $e$ (e) $A_{1} V, \ldots, A_{p-1} V:$ são idempotentes e $A_{p} V$ e não negativa definida.

Pela construção de $\Omega$ e do teorema 1 . pode-se verificar os seguintes fatos:

(i). Considerando o modelo linear ordinário de Gauss-Markov $N_{1}^{Y}=N_{P_{1}}^{\theta}+N_{1}$, isto $\Leftrightarrow Y \cap N\left(X \theta ; \alpha^{2} I_{N}\right)$ com $p^{-1}$ conjuntos paramétricos $C$ podendo naturalmente incluir interaçōes), mais a média geral $\mu$. Neste caso a soma de quadrados total pode ser partida da seguinte forma

$$
\begin{array}{lll}
Y \cdot I_{N} Y & =Y \cdot Y=Y \cdot P_{0} Y+Y \cdot P_{1} Y+---+Y \cdot P_{P} & \text { onde } \\
I_{N}=P_{0}+P_{1}+---+P_{P} & \text { com }
\end{array}
$$




$$
\begin{aligned}
& Y \cdot P_{O} Y=S Q(\mu) \quad Y \cdot P_{P} Y=\text { SQResidual e } \\
& Y P_{i} Y \quad e \quad a \quad \text { S.Q. correspondente do i-ésimo }
\end{aligned}
$$

conjunto paramétrico para $i=1,2, \ldots, p-1$, onde cada forma quadrática $\sigma^{-2} Y P_{i} Y \quad i=0,1,2, \ldots, P$ tem distribuição $x^{2}\left(r\left(P_{i}\right) ; \lambda_{i}\right)$ onde $\lambda_{i}=\theta \cdot X \cdot P_{i} x \theta / 2 o^{2}$ para $i=1,2, \ldots, p-1$, $\lambda_{p}=0$ esão independentes entre si com $\quad \sum_{i=0}^{P} r\left(P_{i}\right)=N$.

Geralmente é isto que se faz na ANOVA usual, permitindo os testes exatos. Nota-se ainda que segundo a classe $\mathfrak{F}$ de matrizes de variâncias e covariancias, basta tomar-se $c_{0}=c_{1}=\ldots=c_{p}=\alpha^{2}$ e tem-se $\quad I_{N}=P_{0}+P_{1}+\cdots+P_{P}$, isto $e$, para $\quad c_{i}=\sigma^{2} \quad(i=1,2, \ldots, p)$ teremos novamente a análise de variancia usual.

(ii). Considerando agora $Y \cap N\left(X \theta ; \alpha^{2} V\right)$, para 0 modelo dado anteriormente, com $V \in \mathbb{F}$.

Sabe-se que a soma de quadrados total é dada por $Y \cdot V^{-1} Y$ e pode ser partida da seguinte maneira $Y \cdot V^{-1} Y=c_{0}^{-1} Y \cdot P_{0} Y+c_{1}^{-1} Y \cdot P_{1} Y+\cdots+c_{P}^{-1} Y \cdot P_{P} Y \quad$ onde $c_{0}^{-1} Y \cdot P_{0}=\operatorname{SQ}(\mu), \quad c_{P}^{-1} Y \cdot P_{P}^{Y}=$ SQResidual $\theta \quad c_{i}^{-1} Y \cdot P_{i}^{Y} \Leftrightarrow$ a soma de quadrados do i-ésimo conjunto paramétrico do modelo, cuja forma quadrática $\left(\sigma^{2} c_{i}\right)^{-1} Y \cdot P Y$ tem

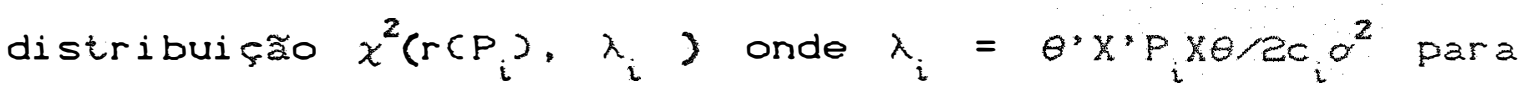
$i=0,1,2, \ldots, p-1$ e $\lambda_{p}=0$. 
20

Para verificar-se que a S. Q. Total dada por Y"V $V^{-1} Y$, basta utilizar-se a transformação $Z=V_{*}^{\prime} Y$ ond $\theta$ $v^{-\frac{1}{2}}=v_{*} v^{\prime} \cdot$

De $V=c_{0} P_{0}+c_{1} P_{1}+\cdots+c_{p} P_{p} \quad$ decorreque

$$
\begin{aligned}
V^{-1} & =V^{-1} V V^{-1}=c_{0} V^{-1} P_{0} V^{-1}+c_{1} V^{-1} P_{1} V^{-1}+\cdots+c_{P} V^{-1} P_{P} V^{-1}= \\
& =c_{0}^{-1} P_{0}+c_{1}^{-1} P_{1}+\cdots+c_{p}^{-1} P_{p} \quad \text { pois }
\end{aligned}
$$

$$
\begin{aligned}
& P_{i} V=c_{i} P_{i} \Rightarrow c_{i}^{-1} P_{i}=P_{i} V^{-1} \\
& V P_{i}=c_{i} P_{i} \Rightarrow c_{i}^{-1} P_{i}=V^{-1} P_{i} \\
& \text { entao } \quad c_{i} V^{-1} P_{i} V^{-1}=V^{-1} P_{i}=c_{i}^{-1} P_{i} \\
& Y V^{-1} Y=c_{0}^{-1} Y P_{0} Y+c_{1}^{-1} Y^{\prime} P_{1} Y+\cdots+c_{P}^{-1} P^{\prime} P_{P} Y .
\end{aligned}
$$




\subsection{MOdelos COM UM FATOR}

\subsubsection{UM FATOR ALEATORIO}

\section{a. Erros Independentes}

Seja o modelo linear, balanceado, de ereitos aleatórios e caracterizado por

$$
y_{i k}=\mu+\alpha_{i}+e_{i k}
$$

para $i=1,2 \ldots, r \quad, k=1,2, \ldots, k$ onde $y_{i k}$ representa a k-ésima observação no $i$-ésimo nivel do fator $\alpha, \mu$ é a módia geral, $\alpha_{i}$ é o efeito do i-ésimo nivel do fator $\alpha e$ $\theta_{i k} e ́$ o erro aleatório devido a observação $y_{i k}$ A expressão "balanceado" refere-se a número igual de observaçбes por casela, conforme SEARLE (1887).

$$
\text { Tomando-se } \alpha_{i} \cap \text { NCO; } \sigma_{\alpha}^{2} \text { e } e_{i k} n \text { NCO; } \alpha_{e}^{2}
$$

independentes, tem-se:

$$
\begin{aligned}
& \operatorname{cov}\left(y_{i k}, y_{i, k^{\prime}}\right)=E\left[\left(y_{i k}-E\left(y_{i k^{\prime}}\right)\left(y_{i^{\prime} k^{\prime}}-E\left(y_{i \cdot k^{\prime}}\right)\right]=\right.\right. \\
& =E\left[\left(\alpha_{i}+e_{i k}\right)\left(\alpha_{i,}+e_{i \cdot k}\right)\right]=E\left(\alpha_{i} \alpha_{i}\right)+E\left(e_{i k} e_{i \cdot k}\right)= \\
& =\left\{\begin{array}{ccc}
\alpha_{e}^{2}+\alpha_{\alpha}^{2} & p / \quad i=i, e k=k^{\prime} \\
\alpha_{\alpha}^{2} & p^{\prime} & i=i, e k \neq k^{\prime} \\
0 & p^{\prime} & i \neq i
\end{array}\right.
\end{aligned}
$$

Logo a matriz de variancias e covariancias para esse modelo pode ser escrita na forma:

$$
\Omega=I_{r}\left[\sigma_{e}^{2} I_{K}+\sigma_{\alpha}^{2} k k_{k}\right]
$$

onde é o produto de Kronecker, e $K_{K}$ é a matriz de elementos unitários multiplicada pelo fator $k^{-1}$, de ordem $k$. 
As somas de quadrados usuais são dadas por:

$\operatorname{sex} \mu=\frac{1}{N} y^{2} . \quad=Y \cdot P_{0} Y$

$\operatorname{soc} \omega=\frac{1}{K} \sum_{i} y_{i}^{2}-\frac{1}{N} y^{2}=Y P_{1} Y$

SQTotal $=\sum_{i k} y_{i k}^{2} \quad=Y \cdot I_{N} Y$

SOResidual $=$ SOTotal $-\left(S Q(\mu)+S Q(\omega)=Y \cdot P_{5} Y\right.$

onde $\quad P_{0}=K_{N}$

$P_{1}=I_{r} \otimes K_{K}-K_{N}$

$P_{5}=I_{N}-I_{r} \otimes K_{K}$

$N=r K \bullet Y$ é o vetor $N$-dimensional de todas as

observaçőes do experimento.

Verifica-se através das propriedades do produto de Kronecker, veja por exemplo IEMMA (1988), dentre outros, que as matrizes $P_{0} P_{1}$ e $P_{5}$ Cprojetores ortogonais) são simstricas e idempotentes tais que $I_{N}=P_{0}+P_{1}+P_{5}$

Tomando $\Omega$ tal que $\Omega \in \Omega$ classe de matrizes positivas definidas, derinida por:

$J=\left\{\Omega: \Omega=c_{0} P_{0}+c_{1} P_{1}+c_{5} P_{5}\right.$, com $\left.c_{0} O_{1} c_{1} O e_{5} c_{5}\right\}$

Entao $\Omega=C_{0} P_{0}+C_{1} P_{1}+C_{5} P_{5}$, onde obtém-se o seguinte sistema de equaçores:

$c_{0} \frac{1}{N}+c_{1} \frac{r-1}{N}+c_{5} \frac{k-1}{K}=\sigma_{0}^{2}+\sigma_{\alpha}^{2}$

$c_{0} \frac{1}{N}+c_{1} \frac{r-1}{N}+c_{5} \frac{-1}{K}=\sigma_{\alpha}^{2}$

$c_{0} \frac{1}{N}+c_{1} \frac{-1}{N}=0$ 
cuja solução dada por

$$
\begin{aligned}
c_{0}=c_{1} & =\sigma_{\theta}^{2}+k \sigma_{\alpha}^{2} \\
c_{5} & =\sigma_{e}^{2}
\end{aligned}
$$

Logo

$$
\begin{aligned}
\Omega & =\left(\sigma_{e}^{2}+k \sigma_{\alpha}^{2}\right)\left(P_{0}+P_{1}\right)+\alpha_{e}^{2} P_{5}= \\
& =\left(\sigma_{\theta}^{2}+k \sigma_{\alpha}^{2}\right) I_{r} \otimes K_{K}+\sigma_{e}^{2}\left(I_{N}-I_{r} \otimes K_{K}\right) \\
& =\sigma_{\theta}^{2} I_{N}+k \sigma_{\alpha}^{2} I_{r} \otimes K_{K}=I_{r} \otimes\left[\sigma_{e}^{2} I_{K}+\sigma_{\alpha}^{2} K K_{K}\right] .
\end{aligned}
$$

E como

$$
\begin{aligned}
P_{i} \Omega & =c_{i} P_{i} \\
P_{i} \Omega P_{j} & =0 \quad \frac{P_{i}}{c_{i}} \Omega=P_{i}
\end{aligned}
$$

decorre que $\quad Y^{\prime} \frac{P_{i}}{C_{i}} Y \cap \chi^{2}\left(r\left(P_{i}\right), \lambda_{i}\right)$

onde

$\lambda_{i}=\frac{1}{2 c_{i}} E\left(Y^{\prime}\right) P_{i} E(Y)$ e såo independentes para $i=0.1 .5$.

\begin{tabular}{|c|c|c|c|c|}
\hline c. $v$. & g. 1 . & S. Q. & Q. M. & $E(Q . M)$. \\
\hline$\mu$ & 1 & $Y \cdot P_{0} Y$ & $Y * P_{0} Y$ & $c_{0}+N \mu^{2}$ \\
\hline$a$ & $r-1$ & $Y \cdot P_{Y} Y$ & $Y>P_{1} Y /(r-1)$ & $c_{1}$ \\
\hline Residuo & $r(x-1)$ & $Y P_{5} Y$ & $Y \cdot P_{5} Y / r(k-1)$ & $c_{5}$ \\
\hline Total & $\mathbf{N}$ & $Y \cdot I Y$ & & \\
\hline
\end{tabular}

As esperanças dos quadrados médios são dadas por:

$E(Q M(\mu))=E\left(Y \cdot P_{0} Y\right)=\operatorname{tr}\left(P_{0} \Omega+E(Y \cdot) P_{0} E(Y)=c_{0}+N \mu^{2}\right.$

$E \operatorname{com}(\omega)=E\left(Y \cdot P_{1} Y\right)=c_{1}$

$E($ CMResiduo $)=E\left(Y \cdot P_{5} Y\right)=c_{5}$

obtendo-se assim o seguinte quadro de análise de variancia.

Quadro 3.1. Análise de variancia para o modelo (3.1) caso a 
de onde decorre que o criterio para o teste de $H_{0}: \sigma_{\alpha}^{2}=0$ $v \approx H_{1}: \sigma_{\alpha}^{2} \neq 0$ pode ser obtido através da estatistica $\operatorname{QH}(\alpha)$

MfResiduos, que sob $H_{0}$ tem distribuiçăo de F central dada por $F\left[r\left(P_{1}\right), r\left(P_{5}\right), 0,0\right]$.

\section{b. Erros não Independentes}

Para o modelo (3.1) considera-se agora o equicorrelacionamento no vetor de erros dado por e $n$ NCO, $\Omega_{1}$ onde $e$ o vetor $N$-dimensional dos erros $\theta$

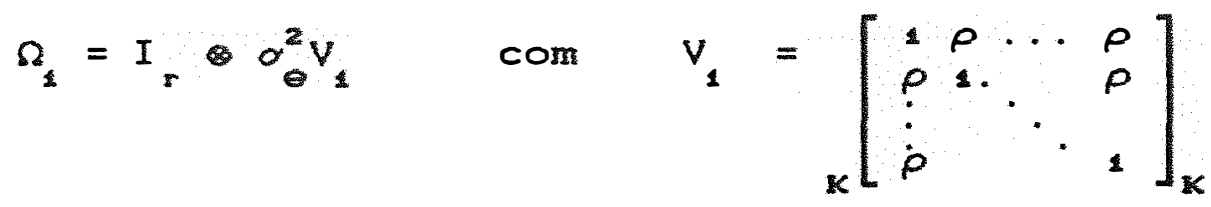

Então por um desenvolvimento analogo a (3.2) obtem-se a matriz $\Omega$ de variáncias e covariáncias do modelo dada por

$$
\Omega=I_{r} \cdot\left[\left(\alpha_{e}^{2}-\infty \alpha_{e}^{2}\right) I_{k}+\left(\infty \alpha_{e}^{2}+\alpha_{\alpha}^{2}\right) K_{k}\right]
$$

Tomando-se $\Omega \in Z$, como reito anteriormente om

(3.3) obtern-se o seguinte sistema de equaçres:

$$
\begin{aligned}
& c_{0} \frac{1}{N}+c_{1} \frac{r-1}{N}+c_{5} \frac{K-1}{K}=\sigma_{e}^{2}+\sigma_{\alpha}^{2} \\
& c_{0} \frac{1}{N}+c_{1} \frac{r-1}{N}+c_{5} \frac{-1}{K}=p \sigma_{e}^{2}+\sigma_{\alpha}^{2} \\
& c_{0} \frac{1}{N}+c_{1} \frac{-1}{N}
\end{aligned}
$$

cuja soluçæo dada por: 


$$
\begin{aligned}
c_{0}=c_{1} & =\sigma_{\theta}^{2}[1+(k-p) \rho]+k \alpha_{\alpha}^{2} \\
c_{s} & =\sigma_{0}^{2}[1-\rho]
\end{aligned}
$$

com $\quad \frac{-1}{k-1}<\rho<1$

Usando-se esses coeficientes torna-se possivel escrever a matriz $\Omega$ na forma anterior, ou seja

$$
\Omega=I_{r}\left[\left(\sigma_{\theta}^{2}-\rho \sigma_{\theta}^{2}\right) I_{K}+\left(\rho \sigma_{\theta}^{2}+\sigma_{\alpha}^{2}\right) \mathrm{KK}_{K}\right] \quad(3.5) .
$$

De (3.4) tem-se que:

$Y \cdot \frac{P_{0}}{C_{0}} Y \cap x^{2}\left(r\left(P_{0}\right), \lambda_{0}\right) \operatorname{com} \lambda_{0}=\frac{1}{Z c_{0}} E(Y,) P_{0} E(Y)$ $Y=\frac{P_{1}}{C_{1}} Y \cap \chi^{2}\left(r\left(P_{1}\right), \lambda_{1}\right)$ com $\lambda_{1}=\frac{1}{2 c_{1}} E(Y,) P_{1} E(Y)$ $Y=\frac{P_{5}}{C_{5}} Y \cap \chi^{2}\left(r\left(P_{5}\right), \lambda_{5}\right)$ com $\lambda_{5}=\frac{1}{2 c_{5}} E(Y *) P_{5} E(Y)$ e são independentes entre si.

Para $\Omega$ dada por (3.5) define-se variável aleatória $F_{15}\left(c_{1} \cdot c_{5}\right)$ dada por

$F_{15}\left(c_{1}, c_{5}\right)=\frac{c_{5}}{c_{1}} \frac{Y^{\prime} P_{1} Y /(r-1)}{Y^{\prime} P_{5} Y / r(K-1)} \quad n F\left[r-1, r(K-1), \lambda_{1}, \lambda_{5}\right]$.

(3.6)

Se $c_{1}=c_{5}$ então $(3.6)$ é a estatistica usual para 0 teste de $H_{0}: \sigma_{\alpha}^{2}=0$. como aconteceu anteriormente considerando-se o vetor de erros independentes.

Entzo se o pesquisador considerar os èros independentes, quando na verdade eles sao equicorrelacionados (basta ver $(3.5)$ ), estará cometendo um erro porque a 
distribuição $F$ por ele utilizada o na verdade proporcional a distribuição $F_{15}\left(c_{1}, c_{5}\right)$. Assim com as somas de quadrados usuais obtém-se 0 seguinte quadro de análise de variancia:

Quadro 3.2. Análise de variancia para o modelo(3.1) caso b.

\begin{tabular}{|c|c|c|c|c|}
\hline c. $v$. & g. 1.1 & S. Q. & Q. $M$. & $E(Q . M)$. \\
\hline$H$ & 1 & $Y \cap P_{0} Y$ & $Y=P_{O} Y$ & $\alpha_{\theta}^{2}[1+(k-1) \rho]+k \sigma_{\alpha}^{2}+N \mu^{2}$ \\
\hline$\alpha$ & $r-1$ & $Y: P_{1} Y$ & $Y \cdot P_{1} Y /(r-1)$ & $\alpha_{e}^{2}[1+(k-1) \rho]+k \alpha_{\alpha}^{2}$ \\
\hline Residuo & $r(k-1)$ & $Y^{\prime} P_{5} Y$ & $Y \cdot P_{5} Y / r(K-1)$ & $\alpha_{\theta}^{2}[1-\rho]$ \\
\hline Total & N & $Y I_{N} Y$ & & \\
\hline
\end{tabular}

Tem-se entao que $P[$ erro tipo I $]=P\left[F \geq F_{\alpha}(r-1, r(K-1))\right]=$ $=P\left[\frac{c_{5}}{c_{1}} F \geq \frac{c_{5}}{c_{1}} F_{\alpha}(r-1 \cdot r(k-1))\right]=$

$=P\left[F_{15}\left(c_{1}, c_{5}\right) \geq \frac{1-P}{1+(k-1) P} F_{\alpha}(r-1, r(K-1))\right] \geqq \alpha$

onde $\alpha \in$ nivel de significancia, supostamente correto, utilizado pelo pesquisador, para testar a hipótese

$$
H_{0}: \alpha_{\alpha}^{2}=0 \text { ve } H_{1}: \alpha_{\alpha}^{2} \neq 0 \text {. Alóm disso }
$$

$$
\begin{aligned}
& \text { P(erro tipo I ) }>\propto \text { se } 0<\rho<1 \\
& \text { P(erro tipo I }=a \text { se } \quad \rho=0 \\
& P(\text { erro tipo } I)<a \text { se } \frac{-1}{k-1}<\rho<0
\end{aligned}
$$

A TABELA A.1.. no apóndice, mostra essas probabilidades para vários valores de $\rho, r, k$ e nivel nominal de significancia 0.05. 
Quanto às probabilidades de erro tipo II. neste caso de modelos aleatórios tem-se:

$P[$ erro tipo II $]=P\left[F_{15}\left(c_{1}, c_{5}\right)<\frac{c_{5}}{c_{1}} F_{\alpha}(r-4, r(k-1))\right]=$ $P\left[F_{15}\left(c_{2} \cdot c_{5}\right)<\left[\frac{1+(k-1) \rho}{1}+\frac{k}{1-\rho} \alpha_{e}^{-2} \alpha_{\alpha}^{2}\right]^{-1} F_{\alpha}(r-1, r(k-1))\right]$

isto sob a hipótese alternativa $H_{1}: \sigma_{\alpha}^{2} \neq 0$. Para ins de exemplificar-se com tabelas, considerar-se-a o valor $\sigma_{e}^{-2} \sigma_{\alpha}^{2}=1$. Neste caso de modelo aleatorio nota-se para - cálculo do poder. que a distribuição $F$ envolvida é central. Essas probabilidades são mostradas na TABELA A.Z. do apéndice.

Para os cálculos das probabilidades dos erros tipo II, utilizou-se a aproximaçăo sugerida por TIKU (1965) da $F$ não central para a $F$ central. Nessa aproximação o autor torna igual os trés primeiros momentos dessas duas distribuiçశes e resolve essas equaçర్s, obtendo novos valores para os graus de liberdade da F central, e em seguida calcula as probabilidades através da funçăo Beta incompleta de PEARSON (1934). Naturalmente, poder-se-ia utilizar outro procedimento (aproximaçăo) para calcular essas probabilidades, como sugere IIKU (1965). Optou-se por esse processo dada a excelente aproximaçăo obtida para as verdadeiras probabilidades calculadas por TANG (1838). Com - auxilio das subrotinas dadas por PRESS et alli (1986) em Fortran. foram obtidos os valores das probabilidades 
através da funçăo Beta incompleta. Para cada caso de equicorrelação foi feito um programa de modo que arquivassem os dados após os cálculos convenientes e com esses arqui vos acionou-se o programa principal, obtendo-se os resultados desejados.

Na elaboração das tabelas das probabilidades dos erros. foram considerados 7 valores para "p" C resguardando a exigencla $\left.c_{i}>0\right)$ e vários valores de $r$, $s$, e $k$, onde $s$ é o número de niveis do segundo fator a ser introduzido, no estudo de modelos com dois fatores. Para as probabilidades dos erros tipo II considerou-se 3 valores para $\lambda, \quad \lambda=0, \lambda=5$ e $\lambda=20$ J. O nivel de significancia nominal (preffixado) considerado foi de 0.05 para todos os casos aqui tratados.

Para a comparação das probabilidades dos erros tipo II obtidas neste trabalho, com outras tabelas dadas por SCHEFFE (1 859), GRAYBILL (1 861 )e outros, deve-se tomar $\left.\varphi^{2}=\lambda / n+1\right)$ onde $n$ é o número de graus de liberdade do numerador, sendo que essas comparaçôes só têm sentido para as colunas em que $p=0$.

$$
\text { Para } \rho=0 \text {. as tabelas fornecem as }
$$

verdadeiras probabilidades dos erros tipos I e II, segundo o nivel de significáncia nominal pré-fixado pelo usuário,e para outros valores de $p$ essas probabilidades são as mais diversas possiveis. 
c. Sugestão de Alguns Procedimentos

\section{Alternativos}

Como o interesse testar a hipótese $H_{0}: \sigma_{\alpha}^{2}=0$ vo $H_{1}: \sigma_{\alpha}^{2} \neq 0$. pode-se contornar esse problema. dentre outras, de duas maneiras relativamente simples.

\section{c.1. Quando $\rho e^{*}$ conhecido}

So $\rho$ é conhecido, pode-se utilizar o metodo da transformação linear no vetor de observaçбes. de forma que os erros do modelo transformado sejam independentes.

Escrevendo o modelo na forma $Y=X \beta+\&$ tal que $Y \cap N \subset \tilde{H}, \Omega \supset$ onde $\Omega$ pode ser dada por $\Omega=\sigma_{e}^{2} V$ com V matriz positiva definida de constantes "conhecidas". ou seja. basta considerar

$$
\sigma^{2} V=\sigma_{e}^{2}\left[I_{r} \theta\left((1-\rho) I_{k}+\left(\rho+\sigma_{e}^{-2} \sigma_{\alpha}^{2}\right) k k_{k}\right)\right]
$$

Como a soma de quadrados total para esse modelo é dada por $Y \cdot V^{-1} Y$, então utiliza-se a tranformaçăo $Z=V_{*} Y$ onde $V^{-1}=V_{*}^{*}$, assim tem-se que o modelo: $Z=V_{*} X+V_{e}=x^{*} \beta+e$ é tal que $Z \cap N\left(\tilde{\mu}_{*} \sigma_{e}^{2}\right), e$ entá a teoria dos modelos lineares ordinários de Gauss-Markov pode ser utilizada. 
Lembrando-se ainda que $\Omega=c_{0} P_{0}+c_{1} P_{1}+c_{5} P_{5}$ tem-se que $\sigma_{e}^{2} V=\sigma_{e}^{2}\left(c_{0}^{*} p_{0}+c_{1}^{*} P_{1}+c_{5}^{*} P_{5}\right)$.

Entao a soma de quadrados total $Y \cdot V^{-1} Y$ pode ser partida da seguinte forma:

$$
\left.\left.\left.Y \cdot V^{-1} Y=C c_{0}^{*}\right)^{-1} Y \cdot P_{0} Y+C c_{1}\right)^{-1} Y \cdot P_{1} Y+C c_{5}\right)^{-1} Y \cdot P_{5} Y
$$

onde

$$
\left(\alpha_{e}^{2} c_{i}^{* 1} Y \cdot P_{i} Y=c_{i}^{-1} Y P_{i} Y \cap x^{2}\left(r\left(P_{i}\right), \lambda_{i}\right)\right.
$$

para $i=0,1,5$ e sao independentes entre si, com $\left(c_{i}^{*}\right)^{-1} Y \cdot P_{i} Y$ representando a verdadeira soma de quadrados. devida ao parámetro em questão.

De forma analoga à anteriores facilmente calcula-se os seguintes valores

$$
\begin{aligned}
c_{0}^{*}=c_{1}^{*} & =(1+(k-1) \rho)+k o_{e}^{-2} \sigma_{\alpha}^{2} \\
c_{5}^{*} & =(1-\rho)
\end{aligned}
$$

Assim monta-se o seguinte quadro de analise de variancia:

Quadro 3.3. Análise de variancia para o modelo(3.1). caso c.1.

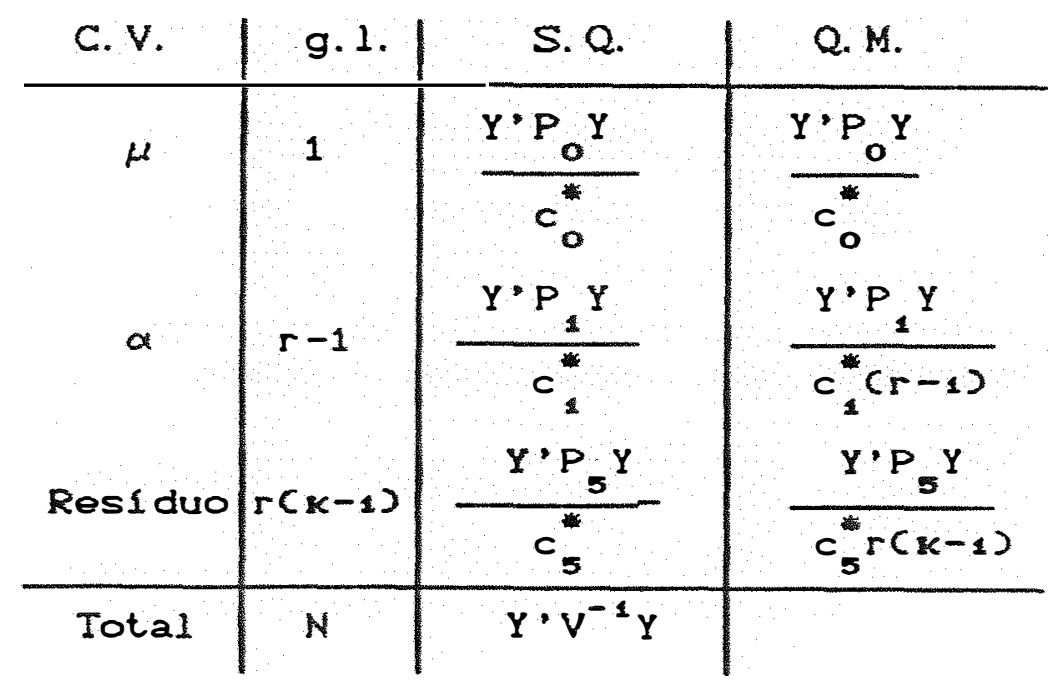


Portanto sob a hipotese $H_{0}: \alpha_{a}^{2}=0$, os coeficientes $\quad c_{0}^{*} c_{1}^{*} c_{5}^{*}$ são numericamente conhecidos

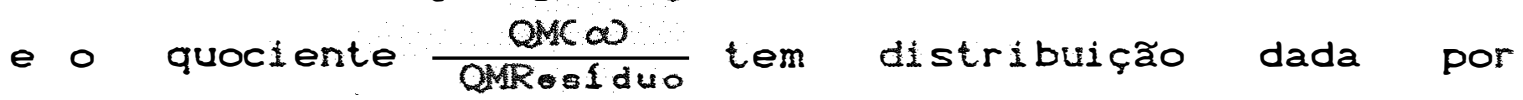
$F[r-1, r(k-1), 0,0]$, permitindo teste exato.

\section{c.2. Quando $\rho e^{*}$ desconhecido}

- procedimento a ser descrito aqui foi sugerido por PERES (1985), para modelos com um fator fixo e balanceado, e não exige que se estime " $p$ ". Consiste numa modificação do planejamento, incluindo neste, répetiçชes independentes de mesmo tamanho e em mesmo número, dentro de cada nivel do rator.

Considerando-se b repetiçơes em cada nível do rator, da seguinte forma:

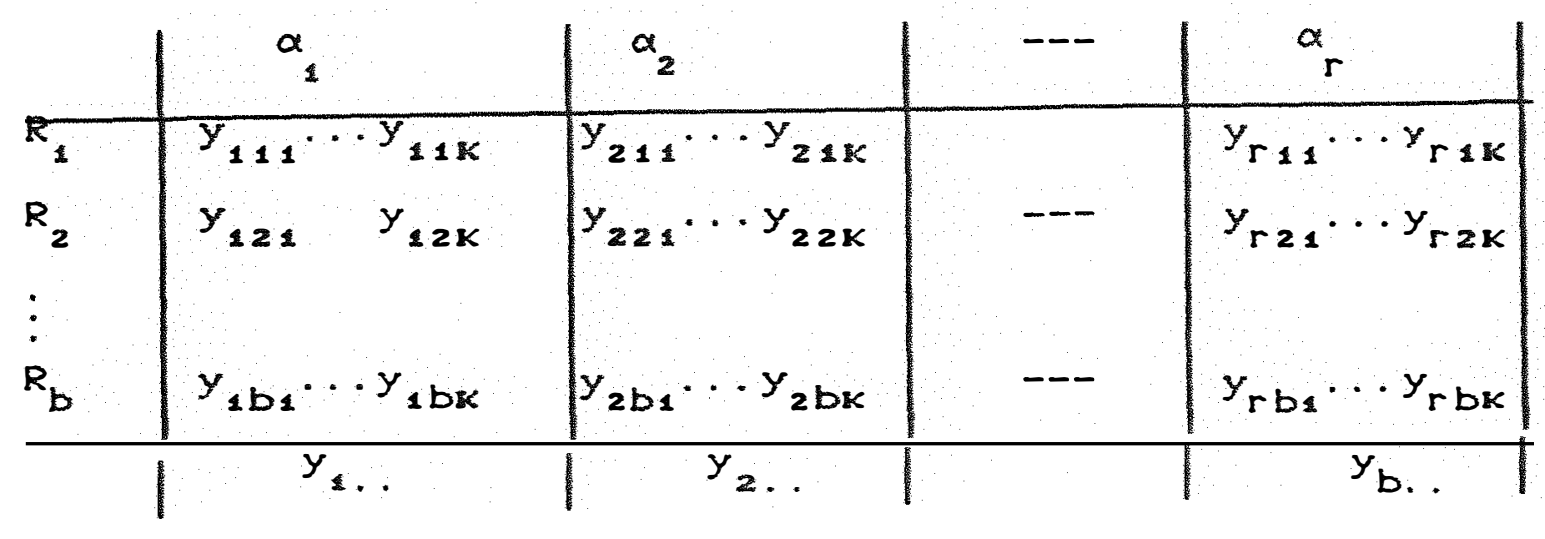

com $\quad y_{i j k}=\mu+\alpha_{i}+R_{j(i)}+e_{i j k}$ sendo a $k$-ésima

observação na j-ésima repetição dentro do i-ésimo nivel do rator. As distribuiçơes são: 


$$
\begin{aligned}
& \alpha_{i} \cap N C O: \sigma_{\alpha}^{2} \text { com } \Omega_{\alpha}=\sigma_{\alpha}^{2} I_{N} \\
& R_{j(i)} \cap \mathrm{NCO} ; \alpha_{R}^{2}{ }^{2} \text { com } \Omega_{R}=\alpha_{R}^{2} I_{N} \\
& \text { - } n \text { NCO: } \Omega_{e}{ }^{2} \text { com } \Omega_{\theta}=I_{r b} \alpha_{\theta}^{2} V_{1}
\end{aligned}
$$

- são independentes entre si para $i=1,2, \ldots, r, j=1,2, \ldots, b, k=1,2, \ldots k, e \quad N=r b k$.

Então tem-se:

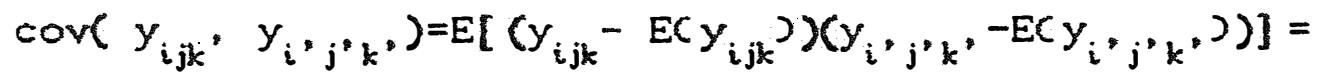
$=E\left[\left(\alpha_{i}+R_{j(i)}+e_{i j k}\right)\left(\alpha_{i}+R_{j,(i,}+e_{i, j}, k^{\prime}\right)\right]=$ $=E\left(\alpha_{i} \alpha_{i}\right)+E\left(R_{j(i)^{\prime}}{ }_{j}^{\prime}(i,)^{\prime}\right)+E\left(e_{i j k} e_{i}{ }^{\prime}{ }^{\prime} k^{\prime}\right)=\ldots$

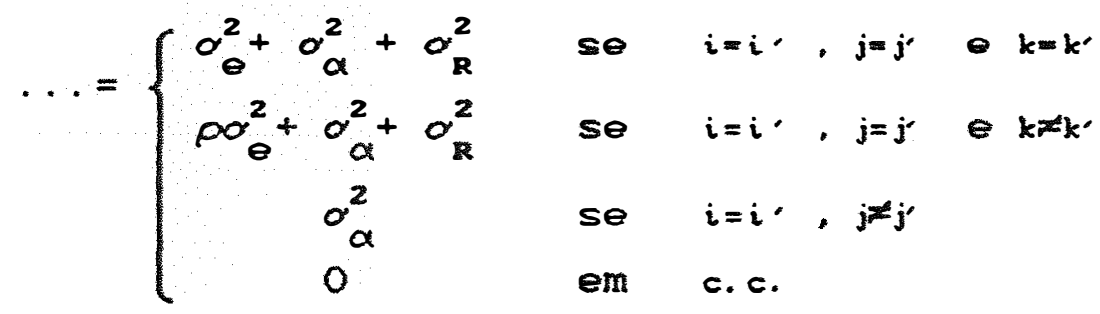

Logo a matriz $\Omega$ do modelo geral é dada por

$$
\Omega=I_{R} \cdot V_{\text {bK }}
$$

onde

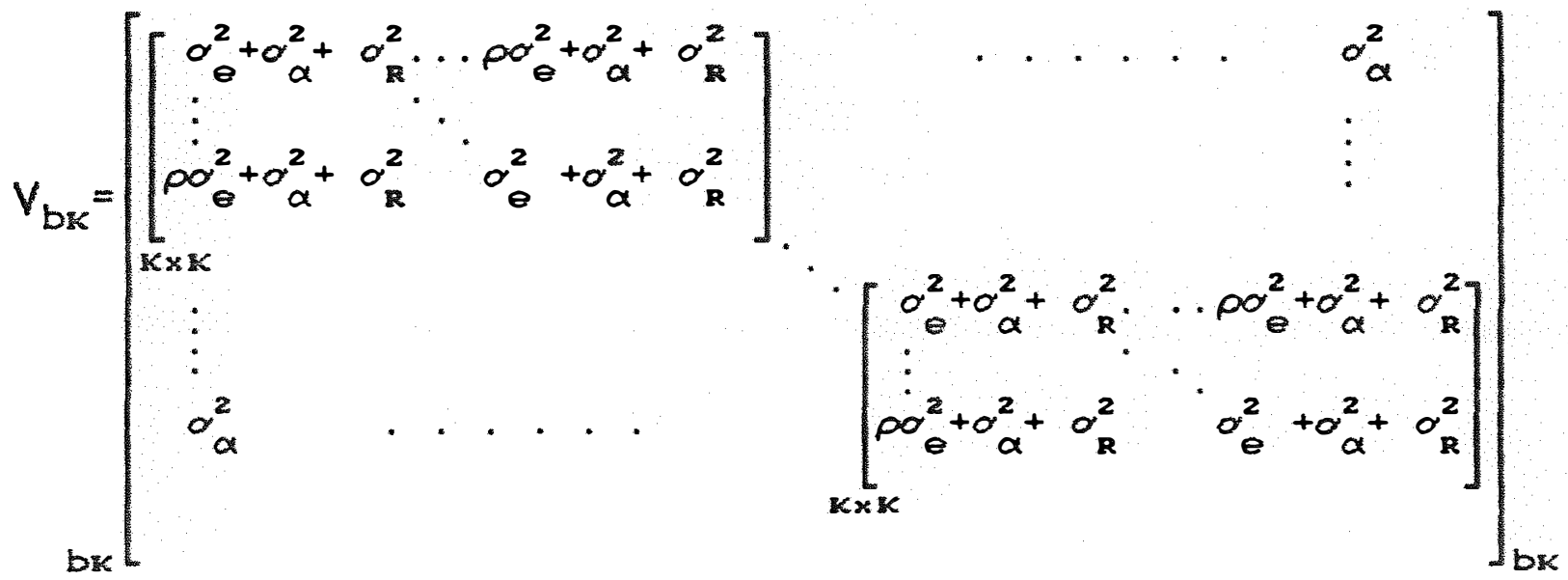


As somas de quadrados são dados por:

$$
\begin{aligned}
& \operatorname{soc} \mu) \quad=\frac{1}{N} y^{2} \cdot \\
& \sec \infty=\frac{1}{b K} \sum_{i} y_{i}^{2} \ldots-\frac{1}{N} y^{2} \ldots=Y \cdot P_{1} Y \\
& S Q\left(R(\omega)=\frac{1}{K} \sum_{i j} y_{i j .}^{2}-\frac{1}{b K} \sum_{i} y_{i}^{2} . .=Y \cdot P_{2} Y\right. \\
& \text { SOResiduo }=Y^{\prime}\left(I_{N}-P_{0}-P_{1}-P_{2} 2 Y \quad=Y \cdot P_{5} Y\right. \\
& \text { SQTotal }=Y^{\prime} I_{N} Y
\end{aligned}
$$

sendo $S Q(R(\alpha)$ ) a soma de quadrados do fator "repetição".

$$
\begin{aligned}
& \text { com } \quad P_{0}=K_{N} \\
& \text { e } r\left(P_{0}\right)=1 \\
& P_{1}=I_{r} \otimes K_{b K}-K_{N} \quad \text { e } r\left(P_{1}\right)=r-1 \\
& P_{2}=I_{r b} \otimes K_{K}-I_{r} \otimes K_{b K} \quad \text { e } r\left(P_{2}\right)=r(b-1) \\
& P_{5}=I_{N}-I_{r b} \otimes K_{K} \quad \Leftrightarrow r\left(P_{5}{ }^{2}=r b(K-1)\right.
\end{aligned}
$$

Escrevendo-se $\quad \Omega=c_{0} P_{0}+c_{1} P_{1}+c_{2} P_{2}+c_{5} P_{5}$, obtem-se o sistema de equaçరes lineares:

$$
\begin{aligned}
c_{0} \frac{1}{N}+c_{1} \frac{r-1}{N}+c_{2} \frac{b-1}{b K}+c_{5} \frac{K-1}{K} & =\sigma_{e}^{2}+\alpha_{\alpha}^{2}+\alpha_{R}^{2} \\
c_{0} \frac{1}{N}+c_{1} \frac{r-1}{N}+c_{2} \frac{b-1}{b K}+c_{5} \frac{-1}{K} & =0 \sigma_{e}^{2}+\alpha_{\alpha}^{2}+\alpha_{R}^{2} \\
c_{0} \frac{1}{N}+c_{1} \frac{r-1}{N}+c_{2} \frac{-1}{b K} & =\sigma_{a}^{2} \\
c_{0} \frac{1}{N}+c_{1} \frac{-1}{N} & =0
\end{aligned}
$$

euja solução : 


$$
\begin{array}{rlrl}
c_{0}=c_{1} & =b k \sigma_{\alpha}^{2}+\sigma_{e}^{2}[1+(k-1) \rho]+k \sigma_{R}^{2} \\
c_{2} & = & \sigma_{e}^{2}[1+(k-1) \rho]+k \sigma_{R}^{2} \\
c_{5} & & \sigma_{e}^{2}[1-\rho]
\end{array}
$$

Utilizando (3.4) pode-se obter 0 seguinte quadro de analise de variáncla:

Quadro 3.4. Análise de variancia para 0 modelo(3.1) acrescido do fator repetição, caso c.Z.

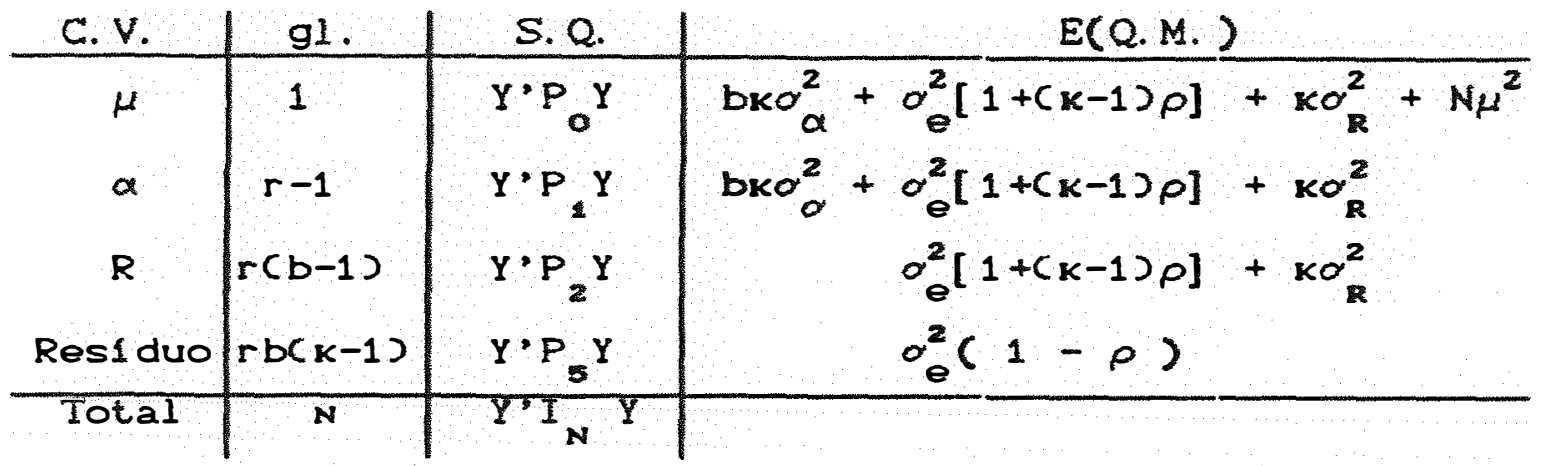

Segundo PERES (1985) tem-se que sob $H_{0}: \sigma_{a}^{2}=0$, uma vez que $c_{1}=c_{2}$, a estatistica

$F_{12}\left(c_{1}, c_{2}\right)=\frac{Y \cdot P_{1} Y /(r-1)}{Y \cdot P_{2} Y / r(b-1)}=F \quad n F[r-1, r(b-1), 0,0]$

e ó a estatistica $F$ usual, isto $\sigma$, mesmo utilizando-se as somas de quadrados usuais, \& possivel neste caso elaborar o teste para essa hipotese $H_{0}: \alpha_{\alpha}^{2}=0$ sem 0 comprometimento do nível de significáncia desejado, mas em contrapartida isso não ocorre para o fator repetição "R". 


\subsubsection{UM FATOR FIXO}

Esse procedimento ja roi desenvolvido para modelos com um fator fixo e balanceado por SCARIANO e DAVENPORT ( 1987 ) más não apresenta uma alternativa para solução do problema, e por PERES ( 1985 ) o qual apresenta como soluçăo alternativa a inclusăo de repetiçơes no planejamento.

Propae-se aqui a generalizaçăo desse procedimento para modelos com um fator fixo e desbalanceado (quanto ao número de observaçбes), cujas correlaçర̃es são afetadas por esse desbalanceamento.

Não será tratado aqui o caso usual de independéncia dos erros, que é um caso particular deste.

a. Os efeitos das violaçơes

Considera-se 0 modelo com um fator sixo caracterizado por: $\quad y_{i k}=\mu+\alpha_{i}+e_{i k}$ para $i=1,2, \ldots, r$ $e k=1,2, \ldots, k_{i} \geq 1$ e $N=k_{1}+k_{2}+\cdots+k_{r}$ é 0 número total de observaçres, sendo $\mu$ a média geral $\alpha_{i} 0$ efeito do i-ésimo tratamento $e e_{i k}$ o erro aleatório năo observável. tal que $E\left(y_{i k}\right)=\mu+\alpha_{i}=\mu_{i}$ e $y_{i k}$ éa k-ésima observação do i-essimo tratamento.

Este modelo ainda pode ser escrito na forma $Y=X \beta+e$ onde $Y \& 0$ vetor de observaçชes dado por: 


$$
\begin{aligned}
Y & =\left[Y_{1}: Y_{2}: \cdots, Y_{r}\right]= \\
& =\left[y_{11} \ldots y_{1 K_{1}} \mid y_{21} \ldots y_{2 K_{2}}, \cdots y_{r_{1}} \cdots y_{r_{r}}\right]
\end{aligned}
$$

o vetor de erros e tal que $E(e)=0$ e $\operatorname{Var}(e)=\Omega$. e $E(Y)=X \beta$.

As somas de quadrados usuais sao dadas por:

$\operatorname{sQ}(\mu)$

$$
=Y \cdot P_{O} Y \text { com } P_{0}=K_{N}
$$

$\operatorname{sQ}(\alpha)$

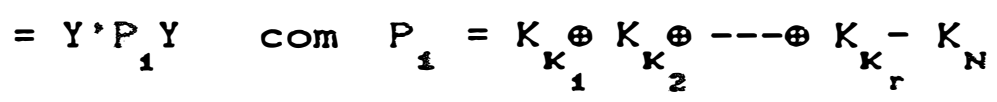

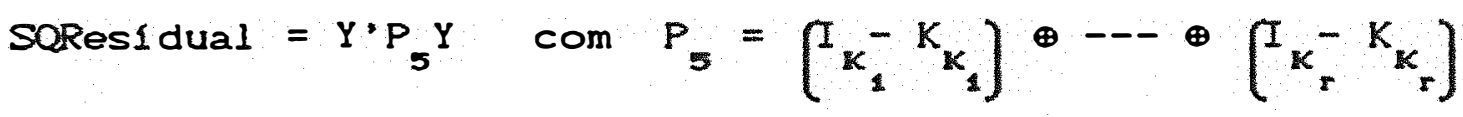

SQTotal $=Y I_{N} Y$ com $I_{N}$ : matriz identidade de ordem $N$ Neste caso. por ser um experimento com número diferente de repetiçbes. deve-se usar a soma direta " $\oplus$ " entre matrizes e não o produto direto "

Através de operaçర̋es simples ( mas exaustivas) pode-se facilmente verificar que os projetores ortogonais $P_{0}, P_{1} \cdot P_{5}$ são matrizes simétricas e idempotentes com

$$
\begin{aligned}
& \operatorname{rank}\left(P_{0}\right)=\operatorname{rank}\left(K_{N}\right)=\operatorname{tr}\left(K_{N}\right)=1 \\
& \operatorname{rank}\left(P_{1}\right)=\operatorname{tr}\left(K_{K_{1}} \oplus--\oplus K_{K_{r}}-K_{N}\right)=r-1 \\
& \operatorname{rank}\left(P_{5}\right)=\operatorname{tr}\left[\left(I_{K_{1}}-K_{K_{1}}\right) \oplus--\oplus\left[I_{K_{r}}-K_{K_{r}}\right)\right]=N-r \\
& \operatorname{rank}\left(I_{N}\right)=N
\end{aligned}
$$

Considerando $\quad \Omega=c_{0} P_{0}+c_{1} P_{1}+c_{5} P_{5}$. para $c_{0}>0, c_{1}>0$ e $c_{5}>0$. E vallidas as distribuiçŏes dada por (3.4) bem como suas independéncias. 
Se $c_{0}=c_{1}=c_{s}=\alpha_{e}^{2}$ ontao $\Omega=\alpha_{0}^{2} \mathrm{~N}$ pois $P_{0}+P_{1}+P_{5}=I_{N}$ entao a analise de variancia o a usual. supondo que os erros sejam independentes e de mesma variancia $\quad \alpha_{e}^{2}$.

Agora. supondo que os erros sejam equicorrelacionados dentro de cada tratamento, ou seja:

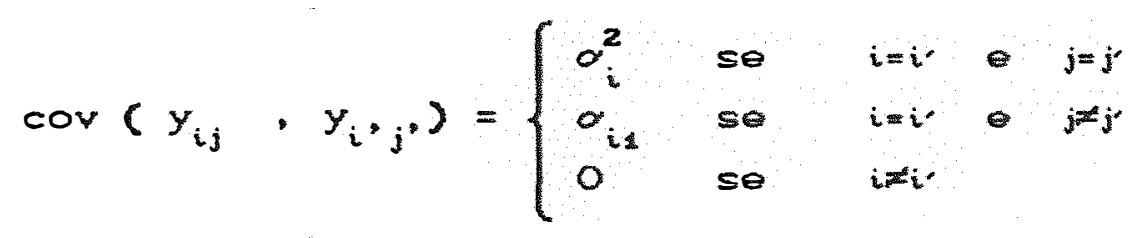
Assim a matriz de variáncias e covariáncias $\Omega$ dada por:

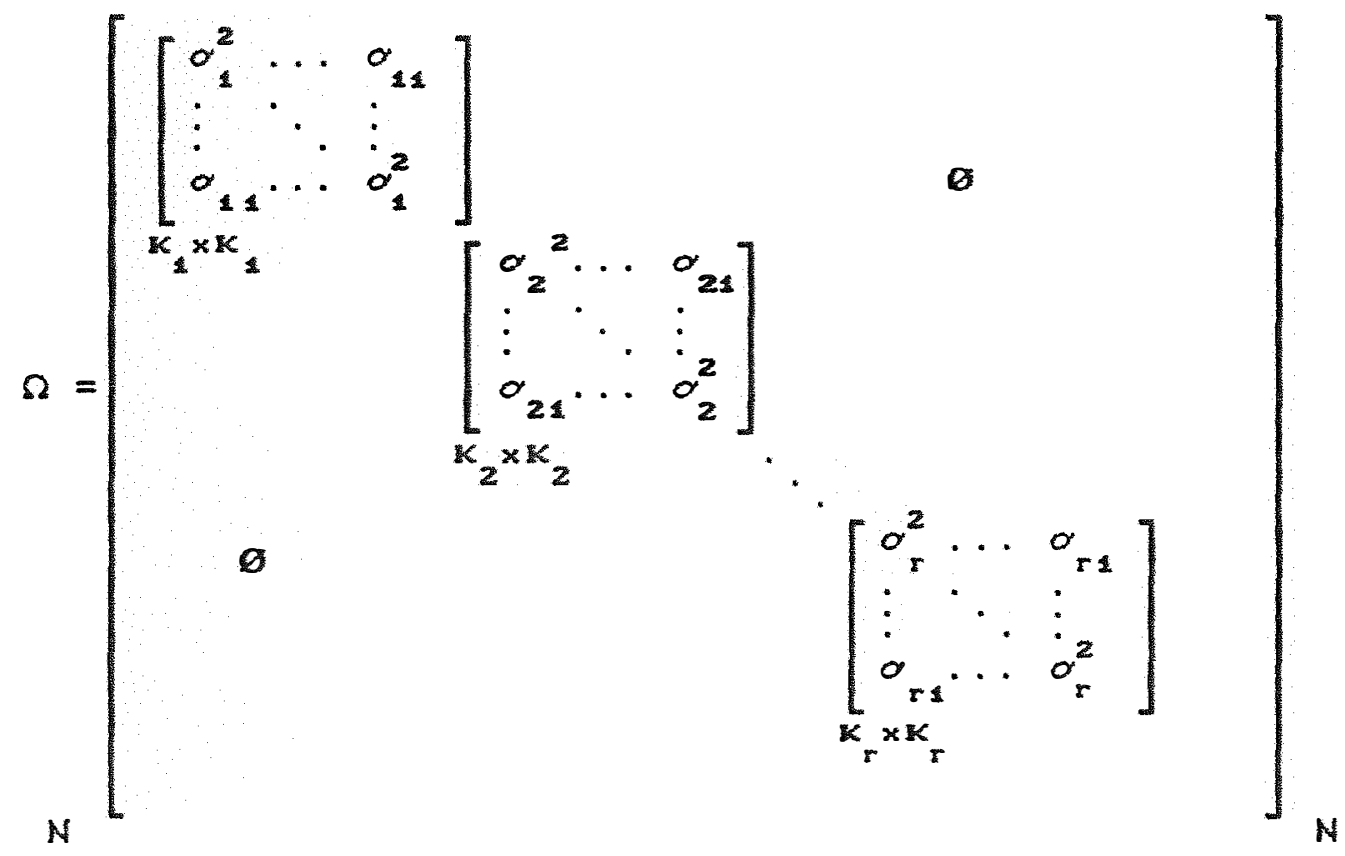

Para determinar $c_{0}, c_{1} \otimes c_{5}$ tais que $\Omega \in \gamma \mid \Omega: \Omega=c_{0} P_{0}+c_{1} P_{1}+c_{5} P_{5}$ com $c_{0}>0, c_{1}>0$ e $\left.c_{5}>0\right\}$, basta tomar 
38

$$
\begin{aligned}
c_{0} P_{0}+c_{1} P_{1}+c_{5} P_{5} & =c_{0} P_{0}+c_{1} P_{1}+c_{5}\left(I_{N}-P_{0}-P_{1}\right)= \\
& =\left(c_{0}-c_{5}\right) P_{0}+\left(c_{1}-c_{5}\right) P_{1}+c_{5} I_{N}=\Omega
\end{aligned}
$$

obtendo o seguinte sistema de equaçós, para cada valor de $i=1,2, \ldots, r$

$$
\begin{array}{ll}
\left(c_{0}-c_{5}\right) \frac{1}{N}+\left(c_{1}-c_{5}\right) \frac{N-K_{i}}{N K_{i}}+c_{5}=o_{i}^{2} \\
\left(c_{0}-c_{5}\right) \frac{1}{N}+\left(c_{1}-c_{5}\right) \frac{N-K_{i}}{N K_{i}}=o_{i 1} \\
\left(c_{0}-c_{5}\right) \frac{1}{N}+\left(c_{1}-c_{5}\right) \frac{-1}{N} & =0
\end{array}
$$

cuja solução para um determinado i é :

$c_{0}=c_{1}=\sigma_{i}^{2}+\sigma_{i 1}\left(k_{i}-1\right) \quad \theta \quad c_{5}=\sigma_{i}^{2}-\sigma_{i 1} \cdot E$ como essa soluçao a válida para todo i. resta-nos a soluçao particular, $\quad \sigma_{i}^{2}=p-q+q \frac{N}{r k_{i}}>0 \Leftrightarrow \sigma_{i 1}=q \frac{N}{r k_{i}}$ fornecendo os valores:

$$
\begin{aligned}
& c_{0}=c_{1}=p+q\left(\frac{N-r}{r}\right) \text { e } c_{5}=p-q, \text { com } \\
& p>0, \frac{-p r}{N-r}<q<p \quad e \frac{-p}{\frac{N}{r k_{i}}-i}<q, \forall i .
\end{aligned}
$$

E pode-se escrever a matriz $\Omega$ na forma

$$
\begin{aligned}
\Omega & =c_{0} P_{0}+c_{1} P_{1}+c_{5} P_{5}=\left[p+q\left(r^{-1}(N-r)\right)\right]\left(P_{0}+P_{1}\right)+(p-q) P_{5} \\
& =(p-q) I_{N}+r^{-1} N q\left(P_{0}+P_{1}\right)=(p-q) I_{N}+r^{-1} N q\left[K_{K_{1}} \oplus--\oplus K_{K_{r}}\right] \\
& =V_{K_{1}} \oplus V_{K_{2}} \oplus--\oplus V_{K_{r}} \quad \text { onde }
\end{aligned}
$$




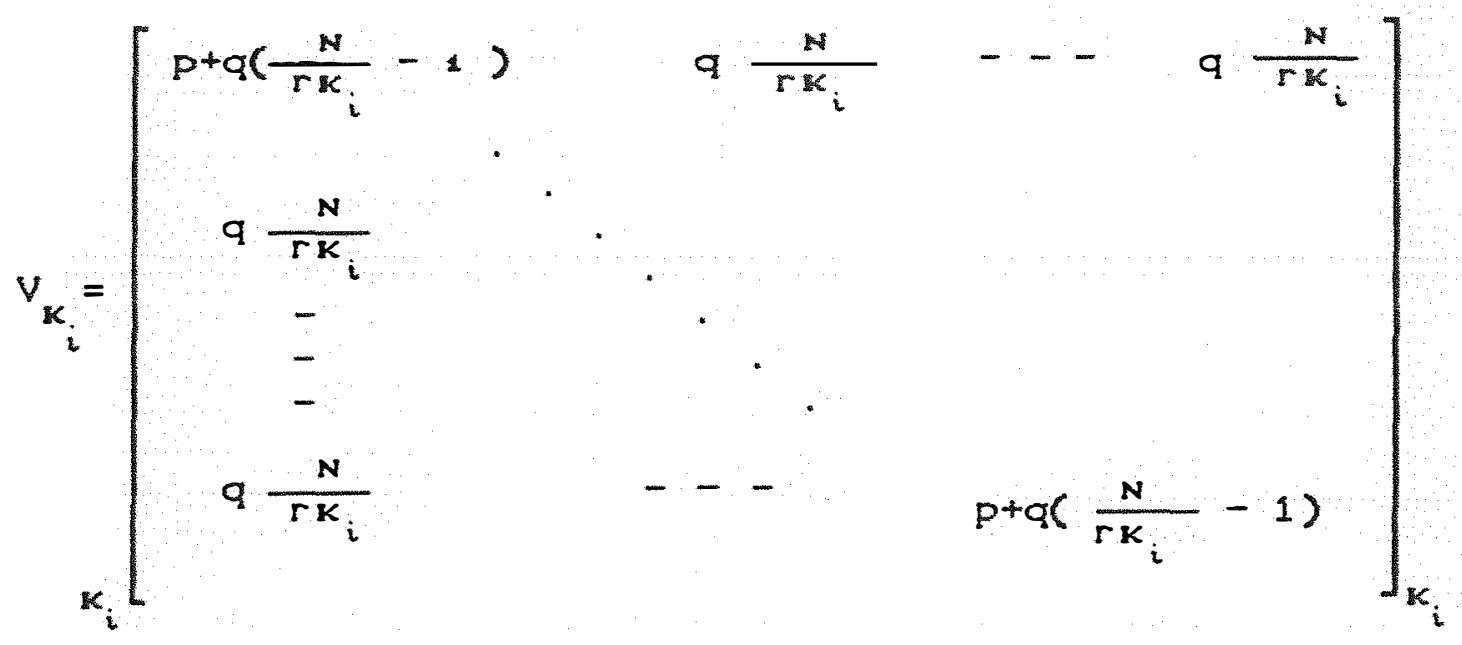

para $i=1,2, \ldots, r$

Então 0 quadro de análise de variância. considerando as restriçöes parametricas $\sum_{i} k_{i} \alpha_{i}=0$ dado por:

Quadro 3.5. Análise de variancia para o modelo dado em 3.2.2.

\begin{tabular}{|c|c|c|c|c|}
\hline C. V. & gl. & S. Q. & Q. $M$. & $E(Q . M)$. \\
\hline$\mu$ & 1 & $Y P_{0} Y$ & $Y, P_{0} Y$ & $c_{0}+N \mu^{2}$ \\
\hline$\alpha$ & $r-1$ & $Y, P Y$ & $Y P_{1} Y$ & $c+1=\alpha^{2}$ \\
\hline & & & $(r-1)$ & 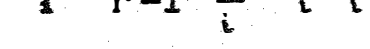 \\
\hline & & & $Y: P_{5} Y$ & \\
\hline & & 5 & $N-r$ & $c_{5}$ \\
\hline Total & $N$ & $Y \cdot Y$ & & \\
\hline
\end{tabular}

o teste $F$ usual supöe a independencia entre as observaçós $C$ isto significa que $q=0$ e $p=\alpha^{2} \mathrm{~J}, \mathrm{um}$ criterio pode ser dado por:

Rojeitar $H_{0}: \alpha_{i}=0$ se $F_{0}=\frac{N-r}{r-1} \frac{Y^{\prime} P_{i} Y}{Y^{\prime} P_{2} Y}=$

$=F_{12}(1,1)>F_{\alpha}(r-1, N-r)$, e coincide com o teste da razão 
de verossimilhança generalizada, sob a condição de que os erros são independentes e identicamente distribuidos com distribuição $N\left(O ; \sigma^{2}\right)$.

Supondo agora que a matriz de variancias e covariáncias seja dada por $\Omega \in$ onde $c_{0}=c_{1}=p+q r^{-1}(N-r)$ e $c_{5}=p-q$. ao invés de $\sigma^{2} I_{N}$. então as probabilidades de erro tipo I para testar a hipotese $H_{0}: \alpha_{i}=0$ ve $H_{i}: \alpha_{i} \neq 0$ para algum i, são na verdade dadas por: $\quad P[$ erro tipo I] = $P\left[F \geq F \alpha_{\alpha}(r-1, N-r)\right]=P\left[c_{1}^{-1} c_{5} F \geq c_{1}^{-1} c_{5} F(r-1, N-r)\right]$ ou seja

$$
\left\{\begin{array}{rrrr}
>\alpha & \text { se } & 0<q<1 & <\operatorname{com} p>q \\
=\alpha & \text { se } & q=0 \\
<\alpha & \text { se } & -(N-r)^{-1} p r<q<0
\end{array}\right.
$$

Essas probabilidades são dadas pela TABELA A. 3. do apéndice, cujos cálculos levam em consideração que

$p=\sigma_{e}^{2}$ e $q=\rho \sigma_{e}^{2} \Rightarrow \frac{c_{5}}{c_{1}}=\frac{1-\rho}{1+\rho r^{-1}(N-r)} \cdot \operatorname{com}$ $P[$ erro tipo I $]=P\left[F_{12}\left(c_{1} \cdot c_{2}\right) \geq \frac{1-\rho}{1+\rho r^{-1}(N-r)} F_{\alpha}(r-1, N-r)\right]$.

Quanto as probabilidades do erro tipo II tem-se:

$P[$ erro tipo II $]=P\left[F_{15}\left[c_{1}, c_{5}, \lambda, 0\right]<c_{1}^{-1} c_{5} F_{\alpha}(r-1, N-r)\right]$. onde $\lambda$ o parámetro de não centralidade dado por $\lambda=2 c_{1}^{-1} E(Y \cdot) P_{1} E(Y)=\left(2 c_{1}(r-1)\right)^{-1} \sum_{i} k_{i} \alpha_{i}^{2}$, essas probabilidades såo dadas pela TABELA A.4. do apéndice. 
Para se obterem as IABELAS A.3. e A.4. . considerou-se $p=\alpha_{e}^{2} \cdot q=p \sigma_{e}^{2} \quad c$ veja comentarios a seguir J, com alguns valores de $\rho \in \lambda$.

Considerando q como sendo a covariancia entre duas observaçơes no mesmo tratamento, e p a variáncia de cada observaçăo, se o experimento fosse bal anceado. Mas como o experimento năo é balanceado, entao a covariancia entre duas observaçoes quaisquer no mesmo tratamento. é afetada pelo rator de proporcionalidade $N\left(r K_{i}\right)^{-1}$ para 0 i-ésimo tratamento, enquanto a variáncia de cada observaçăo - afetada aditivamente pela quantidade $q\left(N\left(r k_{i}\right)^{-1}-1\right)>-p$.

$$
\text { Assim se } \mathrm{p}=\sigma_{e}^{2} \quad e \quad \mathrm{q}=\rho_{e}^{2} \text {, pode-se }
$$

escrever para todo $i=1,2 \ldots \ldots r$

$$
\begin{gathered}
\sigma_{i}^{2}=\sigma_{e}^{2}\left[1+\rho\left(N\left(r k_{i}\right)^{-1}-1\right)\right] \text { e } \sigma_{i 1}=\sigma_{e}^{2} \rho N\left(r k_{i}\right)^{-1} \text { com } \\
c_{0}=c_{1}=\sigma_{e}^{2}\left[1+\rho\left(r^{-1}(N-r)\right)\right] \\
c_{2}=\sigma_{e}^{2}[1-\rho]
\end{gathered}
$$

$$
\text { Pode-se notar que se } q=0 \text { então } p=0 \text { e tem-se }
$$
novamente $\Omega=\alpha^{2} I_{N}$ que $\Leftrightarrow$ a situaçåo usual no caso desbalanceado. Se $k_{i}=k_{2}=--=k_{r}=k$ tem-se $\sigma_{i}^{2}=\sigma^{2}$ e $\quad \sigma_{i 1}=p o^{2}$ que é caso balanceado e estudado pelos autores já citados no inicio do parágrafo 3.2.2. . 
Notatse que os efeitos causados nas probabilidades dos erros tipos I $\Theta$ II não dependem diretamente do desbalanceamento, e sim dos valores de $P$. N e r. embora esse desbalanceamento não possa ser muito acentuado, pois é preciso que $q\left(N\left(r k_{i}\right)^{-1}-1\right)>-p$ ou se $p=\alpha_{e}^{2}$ e $q=p \sigma_{e}^{2}$ entao deverá valer $\rho\left(N\left(r k_{i}\right)^{-1}-1\right)>-1$.

\section{b. Sugestão de um procedimento alternativo}

Com o objetivo de propiciar o teste exato de $H_{0}: \alpha_{i}=0$ vs $H_{1}: \alpha_{i} \neq 0$ para algum $i=1,2, \ldots, r$. propరre-se aqui um procedimento alternativo que consiste em modificar o planejamento, repetindo-o "b" vezes independentemente, incluindo no modelo um novo rator aleatório correspondente à repetiçăo, desde que haja possibilidade por parte do experimentador.

O novo modelo caracterizado por:

$y_{i j k}=\mu+\alpha_{i}+R_{j(i)}+e_{i j k}$ onde $y_{i j k}$ é a k-ésima observação na j-ésima repetição dentro do i-ésimo nível de $\alpha, \operatorname{com} R_{j(i)} e \theta_{i j k}$ variáveis aleatorias independentes para $i=1,2, \ldots, r, j=1,2, \ldots, b \quad k=1,2, \ldots, k_{i}$. alóm disso $R_{j(i)} \cap N\left(O, \sigma_{R}^{2}\right.$. Então a covariancia entre duas observaçơes quaisquer é dada por:

$\operatorname{cov}\left(y_{i j k} \cdot y_{i, j}^{\prime} k^{\prime}\right)=E\left[\left(y_{i j k}-\left(\mu+\alpha_{i}\right)\left(y_{i}^{\prime} j^{\prime} k^{\prime}-\left(\mu+\alpha_{i},\right)\right)\right]=\right.$ 


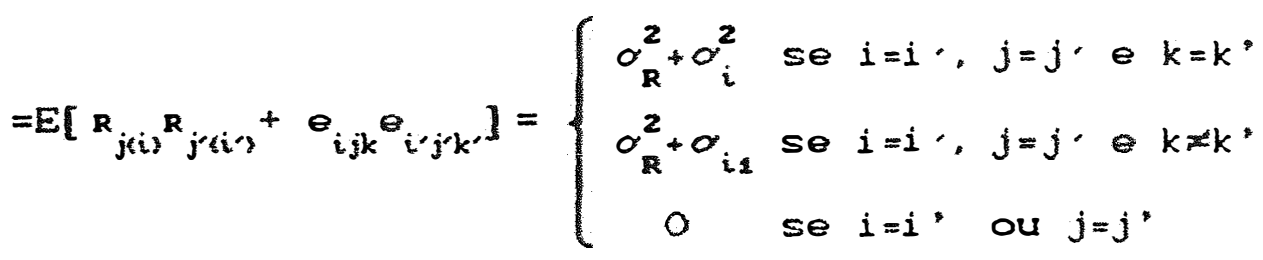

- a matriz $\Omega$ passa a ser dada por

$$
\begin{aligned}
& \left.\left(I_{b} \otimes U_{1}\right) \oplus--I_{b} \bullet U_{r}\right), \text { onde }
\end{aligned}
$$

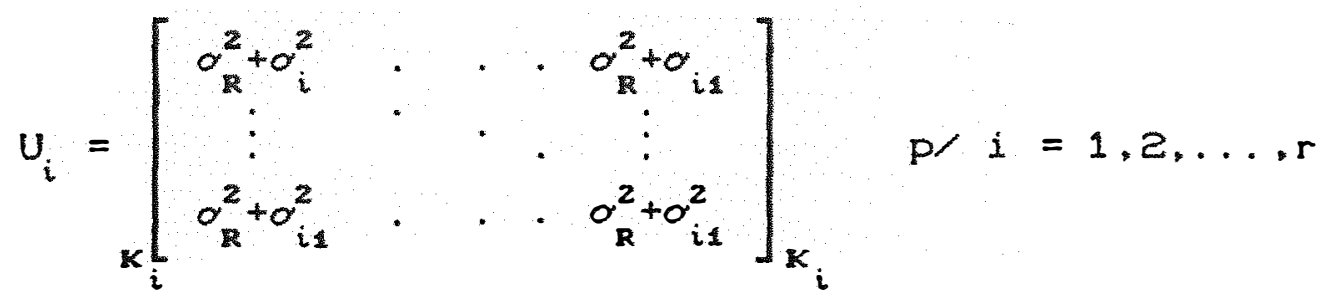

$$
\begin{aligned}
& \text { As somas de quadrados "usuais" săo dadas por: }
\end{aligned}
$$

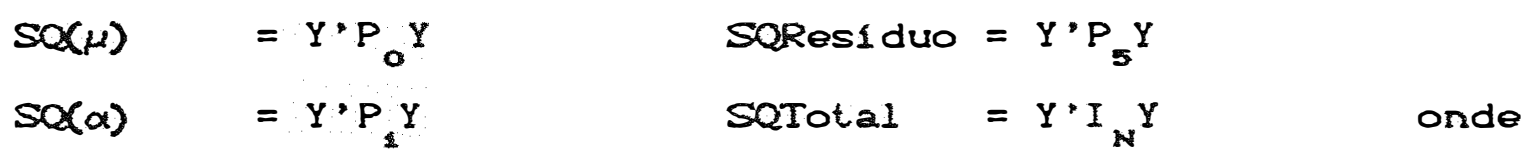

$\operatorname{SQ}\left(R(\alpha)=Y \cdot P_{2} Y\right.$

os projetores ortogonais dados por:

$P_{0}=K_{N}$

$P_{1}=K_{b K_{1}} \oplus K_{b_{k}} \oplus-\cdots K_{b_{k}}-K_{N}$

$P_{2}=\left(I_{b} \otimes K_{K_{1}}\right) \oplus\left(I_{b} \otimes K_{K_{2}}\right) \oplus---\left(I_{b} \otimes K_{K_{r}}\right)-\left(K_{b K_{1}} \otimes K_{b K_{2}} \oplus-\right.$

$\left.--K_{b K_{r}}\right)=\left(I_{b} \otimes K_{K_{1}}-K_{b K_{1}}\right) \oplus--\otimes\left(I_{b} \otimes K_{K_{r}}-K_{b K_{r}}\right)$

$P_{5}=I_{N}-\left(I_{b} \otimes K_{K_{1}}\right) \oplus \cdots\left(I_{b} \otimes K_{K}\right)=$

$=\left(I_{b K_{1}}-\left(I_{b} \otimes K_{K_{1}}\right) \oplus-\cdots\left(I_{b K_{r}}-C I_{b} \otimes K_{K_{T}}\right)\right)$

săo matrizes simetricas e idempotentes, com

$r\left(P_{0}\right)=\operatorname{tr}\left(P_{0}\right)=1$

$r\left(P_{1}\right)=\operatorname{tr}\left(P_{1}\right)=r-1$ 


$$
\begin{aligned}
& r\left(P_{2}\right)=\operatorname{tr}\left(P_{2}\right)=r(b-1) \\
& r\left(P_{5}\right)=\operatorname{tr}\left(P_{5}\right)=N-r b=b\left(\sum_{i}\left(k_{i}-1\right)\right) \\
& r\left(I_{N}\right)=N .
\end{aligned}
$$

Ademais, sendo

$$
\begin{aligned}
& Y=\left[Y_{11}^{\prime} Y_{12}^{\prime} \ldots Y_{1 b}^{\prime} Y_{21}^{\prime} Y_{22}^{\prime} \ldots Y_{2 b}^{\prime} \ldots Y_{r_{1}}^{\prime} Y_{r 2}^{\prime} \ldots Y_{r b}\right] \\
& \text { com } Y_{i j}^{\prime}=\left(y_{i j 1} Y_{i j 2} \cdots Y_{i j K_{i}}\right) \text {, para }\left\{\begin{array}{l}
1=1,2, \ldots, r \\
j=1,2, \ldots, b
\end{array}\right. \\
& \text { e escrevendo-se } \quad \Omega=c_{0} P_{0}+c_{1} P_{1}+c_{2} P_{2}+c_{5} P_{5} \quad \text { ou }
\end{aligned}
$$$$
\text { seja } \Omega=\left(c_{0}-c_{5}\right) P_{0}+\left(c_{1}-c_{5}\right) P_{1}+\left(c_{2}-c_{5}\right) P_{2}+c_{5} I_{N}
$$

obtem-se o seguinte sistema de equações lineares para cada $i=1,2, \ldots, r:$

$$
\begin{aligned}
& \left(c_{0}-c_{5}\right) \frac{1}{N}+\left(c_{1}-c_{5}\right) \frac{N-b k_{i}}{N b k_{i}}+\left(c_{2}-c_{5}\right) \frac{b-1}{b k_{i}}+c_{5}=\sigma_{R}^{2}+\sigma_{i}^{2} \\
& \left(c_{0}-c_{5}\right) \frac{1}{N}+\left(c_{1}-c_{5}\right) \frac{N-b k_{i}}{N b k_{i}}+\left(c_{2}-c_{5}\right) \frac{b-1}{b k_{i}}=\sigma_{R}^{2}+o_{i 1} \\
& \left(c_{0}-c_{5}\right) \frac{1}{N}+\left(c_{1}-c_{5}\right) \frac{N-b k_{i}}{N b k_{i}}+\left(c_{2}-c_{5}\right) \frac{-1}{b k_{i}}=0 \\
& \left(c_{0}-c_{5}\right) \frac{1}{N}+\left(c_{1}-c_{5}\right) \frac{-1}{N}=0
\end{aligned}
$$

cujas soluçơes são dadas por:

$$
\begin{gathered}
c_{0}=c_{1}=c_{2}=k_{i} \sigma_{R}^{2}+\sigma_{i}^{2}+\left(k_{i}-1\right) \sigma_{i 1} \\
c_{5}=\sigma_{i}^{2}-\sigma_{i 1}
\end{gathered}
$$

para todo $i=1,2, \ldots, r, \operatorname{com} \sigma_{i}^{2} \odot \sigma_{i 1}$ dados por

$$
\sigma_{i}^{2}=p-q+q \frac{N}{r k_{i}}>0 \quad \theta \quad \sigma_{i 1}=q \frac{N}{r k_{i}}
$$




$$
\begin{aligned}
c_{0}=c_{1}=c_{2} & =\frac{N}{b r} \sigma_{R}^{2}+p+q\left(\frac{N}{r}-1\right) \\
c_{5} & =p-q
\end{aligned}
$$

satisfazendo $\sigma_{R}^{2}>0: P>0$

$$
\frac{-1}{N-r}\left[r p+\frac{N}{b} o_{R}^{2}\right]<q<p .
$$

As suposiçores de normalidade dos erros continuam válidas, alóm disso as distribuiçres das formas quadráticas $Y \cdot P_{i} Y$, para $i=0,1,2,5$ são independentes com distribui ção de qui-quadrado.

$$
\text { Tem-se que } \begin{aligned}
E\left(Y^{\prime} P_{i} Y\right) & =\operatorname{tr}\left(P_{i} \Omega\right)+E\left(Y^{\prime}\right) P_{i} E(Y) \\
& =r\left(P_{i}\right) c_{i}+E\left(Y^{\prime}\right) P_{i} E(Y) .
\end{aligned}
$$

As esperanças dos quadrados medios são dadas

por :

$$
\begin{array}{ll}
E(\operatorname{ON}(\mu)) & =c_{0}+N\left(\mu+\frac{1}{N} \sum_{i} k_{i} \alpha_{i}\right)^{2} \\
E(\operatorname{OM}(\alpha)) & =c_{1}+\frac{b}{r-1} \sum_{i} k_{i}\left(\mu+\alpha_{i}\right)^{2}-\frac{N}{r-1}\left(\mu+\frac{1}{N} \sum_{i} k_{i} \alpha_{i}\right)^{2} \\
E(\operatorname{OM}(R)) & =c_{2} \\
E(\operatorname{OMR} \operatorname{Res} 1 \text { duo }) & =c_{5}
\end{array}
$$$$
\text { e sob a restriçăo } \quad \sum_{i} k_{i} \alpha_{i}=0 \text { " tem-se: }
$$

$$
\begin{array}{ll}
E(O M(\mu)) & =c_{0}+N \mu^{2} \\
E(O M(\alpha)) & =c_{1}+\frac{b}{r-1} \sum_{i} k_{i} \alpha_{i}^{2} \\
E(Q N(R)) & =c_{2} \\
E(O M R D e l \text { dUO }) & =c_{5}
\end{array}
$$

Assim o quadro de análise de variancia fica: 
Quadro 3.6. Analise de variancia para o modelo dado em 3.2.2. acrescido do fator repetição.

\begin{tabular}{|c|c|c|c|}
\hline c. V. & 91. & S. Q. & ECQ.M. \\
\hline$\mu$ & 1 & $Y P_{0} Y$ & $c_{0}+N \mu^{2}$ \\
\hline$\alpha$ & $r-1$ & $Y^{\prime} P_{1} Y$ & $c_{i}+\frac{b}{r-1} \sum_{i} k_{i} \alpha_{i}^{2}$ \\
\hline$R$ & $r(b-1)$ & $Y^{\prime} P_{2} Y$ & $c_{2}$ \\
\hline Residuo & $N-r b$ & $Y \cdot P_{5} Y$ & $c_{5}$ \\
\hline Total & $N$ & $Y \cdot I_{N} Y$ & \\
\hline
\end{tabular}

e como $c_{0}=c_{1}=c_{2}$, então a hipotese $H_{0}: \alpha_{i}=0$ pode ser testada sem comprometer as probabilidades dos erros tipo I e tipo II, uma vez que sob $H_{0}$ a estatistica $\operatorname{QN}(\alpha)$ / $Q M(R)$ tem distribuiçăo usual dada por

$$
\begin{aligned}
& F_{12}(1,1) \cap F[r-1, r(b-1), 0,0] . \\
& \text { Os comentarios feitos no final do Item }
\end{aligned}
$$

3.2.2.a. também valem aqui, $\theta$ ainda se $p=\sigma_{e}^{2}$ e $q=\rho \alpha_{e}^{2}$, tem-se:

$$
\begin{gathered}
c_{0}=c_{1}=c_{2}=\frac{N}{b r} \alpha_{R}^{2}+\sigma_{e}^{2}\left[1+\rho\left(\frac{N}{r}-1\right)\right] \\
c_{5}= \\
\sigma_{e}^{2}[1-\rho]
\end{gathered}
$$

onde $\quad N=b \sum_{i} k_{i}$.

$$
\text { Se } k_{1}=k_{2}=\ldots=k_{r}=k \text { oxperimento } 6
$$
bal anceado com $\alpha_{i}^{2}=\alpha^{2} \quad e \quad \sigma_{i 1}=\rho \alpha^{2}$, cuja solução \& a mesma já estudada por PERES (1985) e SCARIANO DAVENPORT (1987). 


\subsection{Modelos com Dois fatores fixos e Balanceados}

\subsubsection{MODELOS SEM INTERAÇÃO}

\section{a. Erros independentes}

Consideremos o modelo linear de efeitos fixos e balanceados, caracterizado por:

$y_{i j k}=\mu+\alpha_{i}+\beta_{j}+e_{i j k} \quad$ para $i=1,2, \ldots, r$ $j=1,2, \ldots, s \quad k=1,2, \ldots, k, \quad N=r s k$, onde $\mu$ é média geral, $\alpha_{i}$ o efeito do $i$-ésimo nivel do fator $\alpha, \beta_{j}$ o efeito do j-ésimo nivel do fator $\beta \theta \theta_{i j k} \Leftrightarrow$ - erro aleatório devido à observação $y_{i j k}$.

Como os fatores são fixos e e $n N(0, \Omega)$ então $E\left(y_{i j k}\right)=\mu+\alpha_{i}+\beta_{j}=\mu_{i j}$ para todo $i, j, e k$. No modelo usual os erros são independentes $\theta$ a matriz $\Omega \Leftrightarrow$ do tipo $\Omega=\sigma^{2} I_{N}$, e as somas de quadrados são dadas por:

$$
\begin{aligned}
& S Q(\mu)=Y \cdot P_{0} Y=\frac{1}{N} y^{2} \ldots \\
& \operatorname{soc} \alpha=Y \cdot P_{1} Y=\frac{1}{s K} \sum_{i}^{2} y_{i .}-\frac{1}{N} y^{2} \ldots \\
& \operatorname{SQ}(\beta)=Y \cdot P_{2} Y=\frac{1}{\Gamma K} \sum_{j} y^{2} \cdot j .-\frac{1}{N} y^{2} \ldots \\
& \text { SQResiduo }=Y \cdot P_{5} Y
\end{aligned}
$$


$\operatorname{com} P_{O}=K_{N}$

$P_{i}=I_{r} \otimes K_{S K}-K_{N}=\left[I_{r}-K_{r}\right] \otimes K_{S K}$

$P_{2}=K_{r} \otimes I_{S} \otimes K_{K}-K_{N}=K_{r} \otimes\left[I_{r}-K_{S}\right] \otimes K_{K}$

$P_{5}=I_{N}-\left(P_{0}+P_{1}+P_{2}\right)=I_{N}-I_{r} \otimes K_{S K}-K_{r} \otimes I_{S} \otimes K_{K}+K_{N}$

e $Y=\left[Y_{11}^{\prime} Y_{12}^{\prime} \cdots Y_{1 S}^{\prime} Y_{21}^{\prime} Y_{22}^{\prime} \ldots Y_{2 S}^{\prime} \cdots Y_{r_{1}}^{\prime} Y_{r_{2}}^{\prime} \ldots Y_{r S}^{\prime}\right]^{\prime}$ onde $Y_{i j}=\left(Y_{i j 1} Y_{i j 2}--y_{i j k}\right)$ para

$$
i=1,2, \ldots, r \text { e } j=1,2, \ldots, s
$$

Tem-se que os projetores ortogonais $\mathrm{P}_{0}, \mathrm{P}_{1}$, $P_{2} \ominus P_{5}$ são matrizes simétricas, idempotentes e $P_{0}+P_{1}+P_{2}+P_{5}=I_{N}$ isto 6 as matrizes $P_{0}, P_{1}, P_{2} e$ $\mathrm{P}_{5}$ geram quatro sub-espaços ortogonais do espaco N-dimensional, com dimensöes:

$$
\begin{aligned}
& r\left(P_{0}\right)=1 \\
& r\left(P_{1}\right)=r-1 \\
& r\left(P_{2}\right)=s-1 \\
& r\left(P_{5}\right)=N-r-s+1
\end{aligned}
$$

Tomando-se $\Omega \in \mho=1 \Omega: c_{0} P_{0}+c_{1} P_{1}+c_{2} P_{2}+c_{5} P_{5}=\Omega \mathrm{com}$

$$
\left.c_{i}>0 \text { p } i=0,1,2,5\right\}
$$

obtem-se o seguinte sistema de equaçoes lineares:

$$
\begin{aligned}
& \left(c_{0}-c_{5}\right) P_{0}+\left(c_{1}-c_{5}\right) P_{1}+\left(c_{2}-c_{5}\right) P_{2}+c_{5} I_{N}=\Omega \\
& \left(c_{0}-c_{5}\right) \frac{1}{N}+\left(c_{1}-c_{5}\right) \frac{r-1}{N}+\left(c_{2}-c_{5}\right) \frac{5-1}{N}+c_{5}=o^{2} \\
& \left(c_{0}-c_{5}\right) \frac{1}{N}+\left(c_{1}-c_{5}\right) \frac{r-1}{N}+\left(c_{2}-c_{5}\right) \frac{5-1}{N}=0 \\
& \left(c_{0}-c_{5}\right) \frac{1}{N}+\left(c_{1}-c_{5}\right) \frac{r-1}{N}+\left(c_{2}-c_{5}\right) \frac{-1}{N} \\
& \left(c_{0}-c_{5}\right) \frac{1}{N}+\left(c_{1}-c_{5}\right) \frac{-1}{N}+\left(c_{2}-c_{5}\right) \frac{5-1}{N} \\
& \text { obtendo-se } \quad c_{0}=c_{1}=c_{2}=c_{5}=\sigma^{2}
\end{aligned}
$$


Logo $E\left(Y \cdot P_{i} Y\right)=\operatorname{tr}\left(\cdot P_{i} \Omega\right)+E\left(Y^{*}\right) P_{i} E(Y)=$

$$
\begin{aligned}
& =c_{i} \operatorname{tr}\left(P_{i}\right)+\tilde{\mu} \cdot P_{i} \tilde{\mu}= \\
& =\sigma^{2} r\left(P_{i}\right)+\tilde{\mu} \cdot P_{i} \tilde{\mu}
\end{aligned}
$$

onde $\tilde{\mu}^{\prime}=\left[\tilde{\mu}_{11}^{\prime} \tilde{\mu}_{12}^{\prime} \cdots \tilde{\mu}_{1 s}^{\prime} \cdots \tilde{\mu}_{r_{1}}^{\prime} \tilde{\mu}_{r_{2}}^{\prime} \cdots \tilde{\mu}_{r s}^{\prime}\right]$

$$
\tilde{\mu}_{i j}^{\prime}=\left[\mu_{i j} \mu_{i j} \ldots \mu_{i j}\right] \text { de dimensão k. (3.10) }
$$

Assim a análise de variáncia é a usual, e sob

as restriçơes paramétricas $\sum_{i} \alpha_{i}=\sum_{j} \beta_{j}=0$,

os testes para as hipóteses dos efeitos principais $H_{0}: \alpha_{i}=0$ e $H_{0}: \beta_{j}=0$ são feitos em relação ao residuo e mantém o nivel de significáncia desejado. Assim, tem-se o quadro de análise de variáncia dado a seguir:

Quadro 3.7. Análise de variancia para o modelo (3.7) caso a.

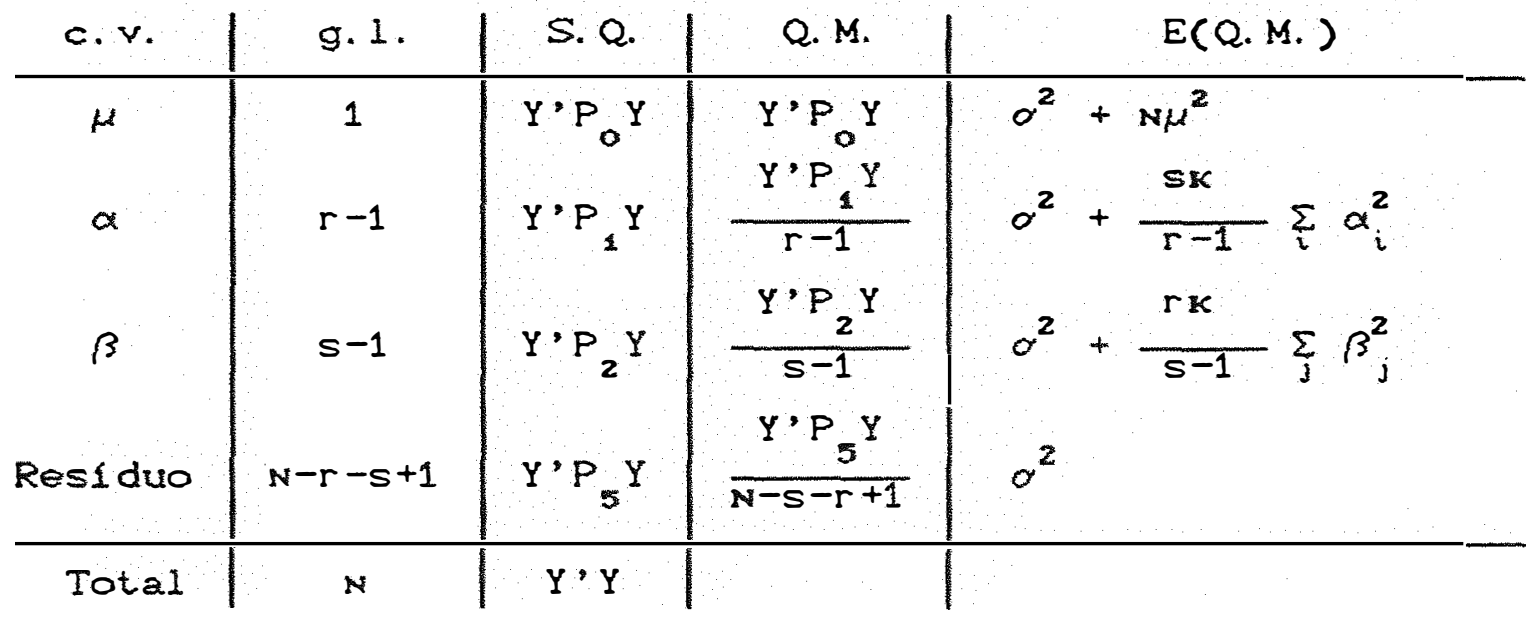

b. Erros não independentes

Iratar-se-á de dois casos de equicorrelação que se supơem serem os mais comuns. 
b.1. Equi correlação das observaç̋̃es na mesma casela

Neste caso a covariancia entre duas observaçסes é dada por:

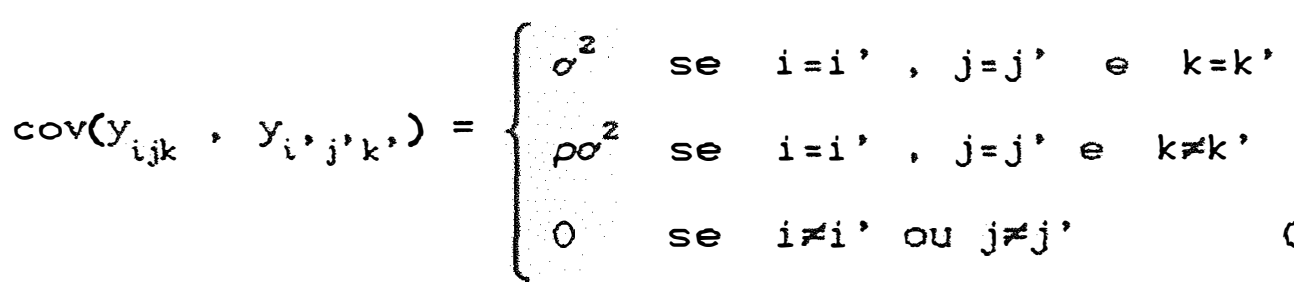

Assim pode-se escrever $\Omega=I_{\text {rs }} \otimes V$ onde

$$
V=\sigma_{K}^{2}\left[\begin{array}{lllll}
1 & 0 & \cdots & \cdots \\
\rho & 1 & \ddots & \cdots & 1 \\
\vdots & \vdots & \cdots & \vdots \\
\rho & 1 & \ddots & \cdots & 1
\end{array}\right]_{K}
$$

com $\quad \Omega \in \mathbb{Z}$ isto e $\quad \Omega=c_{0} P_{0}+c_{1} P_{1}+c_{2} P_{2}+c_{5} P_{5}=$

$$
=\left(c_{0}-c_{5}\right) P_{0}+\left(c_{1}-c_{5}\right) P_{1}+\left(c_{2}-c_{5}\right) P_{2}+c_{5} I_{N}
$$

onde $P_{0}, P_{1}, P_{2} \in P_{5}$ sao dadas por (3.8), obtendo-se 0 seguinte sistema de equaçôs lineares:

$$
\begin{aligned}
& \left(c_{0}-c_{5}\right) \frac{1}{N}+\left(c_{1}-c_{5}\right) \frac{r-1}{N}+\left(c_{2}-c_{5}\right) \frac{5-1}{N}+c_{5}=0^{2} \\
& \left(c_{0}-c_{5}\right) \frac{1}{N}+\left(c_{1}-c_{5}\right) \frac{r-1}{N}+\left(c_{2}-c_{5}\right) \frac{5-1}{N}=00^{2} \\
& \left(c_{0}-c_{5}\right) \frac{1}{N}+\left(c_{1}-c_{5}\right) \frac{r-1}{N}+\left(c_{2}-c_{5}\right) \frac{-1}{N}=0 \\
& \left(c_{0}-c_{5}\right) \frac{1}{N}+\left(c_{1}-c_{5}\right) \frac{-1}{N}+\left(c_{2}-c_{5}\right) \frac{5-1}{N}=0
\end{aligned}
$$


cuja solução é:

$$
\begin{aligned}
& c_{1}=\sigma^{2}\left[\rho\left(\frac{N}{r}-1\right)+1\right] \\
& c_{2}=\sigma^{2}\left[\rho\left(\frac{N}{S}-1\right)+1\right] \\
& c_{0}=\sigma^{2}\left[\rho\left(\frac{N}{r}+\frac{N}{S}-N-1\right)+1\right] \\
& c_{5}=\sigma^{2}[1-\rho]
\end{aligned}
$$

e como $c_{i}>0$ para $i=0,1,2,5$ então $p=0$ ou $p>-(r k+s k-N-1)^{-1}=-(k(r+s-r s)-1)^{-1}$, o qual é sempre positivo.

$$
\text { Como } \begin{aligned}
E\left(Y^{\prime} P_{i} Y\right) & =\operatorname{tr}\left(P_{i} \Omega\right)+E\left(Y^{\prime}\right) P_{i} E(Y)= \\
& =c_{i} r\left(P_{i}\right)+\tilde{\mu}^{\prime} P_{i} \tilde{\mu}
\end{aligned}
$$

onde $\tilde{\mu}^{\prime} \Leftrightarrow$ dado por $(3.10), e$ considerando as restriç̧⿸s dadas por (3.11) obtem-se o quadro de analise de variancia:

Quadro 3.8. Analise de variancia para o modelo (3.7) caso b. 1 .

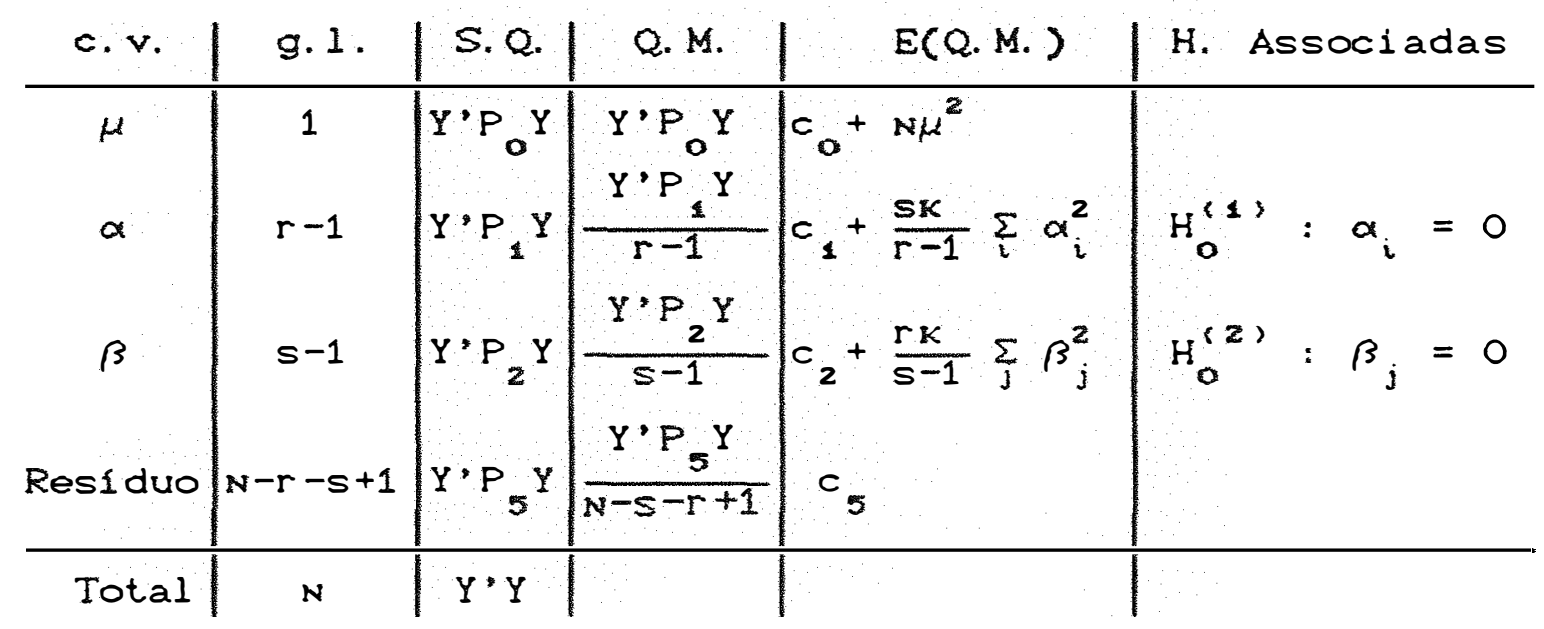

Assim a estatistica usual para testar-se a hipótese $H_{0}: \alpha_{i}=0$ vo $H_{1}: \alpha_{i} \neq 0$ para algum $i=1,2, \ldots, r$ é dada por:

$\left.F_{15}\left(c_{1}, c_{5}\right)=F_{15}\left(\sigma^{2}\left[\alpha \frac{N}{r}-1\right)+1\right] ; \sigma^{2}[1-p]\right)=$ 


$$
=\frac{1-\rho}{1+\alpha\left(\frac{N}{r}-1\right)} F\left[r-1, N-r-s+1 ; \lambda_{1}, 0\right]
$$

e sob a hipótese $H_{0}$, tem-se $\lambda_{1}=0$, então $P($ erro tipo $I)=$ $=P\left(F_{15}\left(c_{1}, c_{2}\right) \geq \frac{1-\rho}{\left.1+\alpha \frac{N}{r}-1\right)} F_{\alpha}(r-1, N-r-s+1) \geqslant \alpha\right.$

No teste de $H_{0}: \beta_{j}=0$ vo $H_{1}: \beta_{j} \neq 0$ para algum $j=1,2, \ldots, s$, tem-se a estatistica:

$\left.F_{25}\left(c_{2}, c_{5}\right)=F_{25}\left(\sigma^{2}\left[\rho \frac{N}{r}-1\right)+1\right], \sigma^{2}[1-\rho]\right)=$ $=\frac{1-\rho}{1+\rho\left(\frac{N}{s}-1\right)} F\left[s-1, N-r-s+1 ; \lambda_{2}, 0\right]$, esob $H_{0}$ tem-se $\lambda_{2}=0$, ent ão

$P($ errotipo I) $=$

$=P\left(F_{25}\left(c_{2}, c_{5}\right) \geq \frac{1-\rho}{\left.1+\alpha \frac{N}{S}-1\right)} F_{\alpha}(s-1, N-r-s+1)\right) \geqslant \alpha$

onde $a$ é nivel de significancia, supostamente correto, utilizado pelo experimentador, para testar cada uma das hipoteses citadas, com

$$
\begin{aligned}
& P(e r r o t i p o I)>\alpha \text { se } P>0 \\
& P(e r r o t i p o I)=\alpha \text { se } \rho=0 \\
& P(e r r o t i p o I)<\alpha \text { se } P<0
\end{aligned}
$$

então se $\rho \neq 0$, nenhum dos testes pode ser feito de maneira "usual" , com a exatidão do nivel de significancia pré-fixado $\alpha$. A TABELA A.5. do apéndice fornece as probabilidades dos erros tipo I para as duas hipoteses $H^{\langle 1\rangle} \odot H^{(2)}$, segundo valores de $\rho, r, s, k$ e nivel 
nominal de significancia 0,05 .

As próbilidades do erro tipo II também são comprometidas.

$$
\text { Para a hipótese } H_{0}: a_{i}=0 \text { ve } H_{1}: a_{i} \neq 0
$$

para algum $i=1,2, \ldots, r$, tem-se:

P(erro tipo II $=P\left(F_{15}\left[c_{1}, c_{5}, \lambda_{1}, O\right]>\frac{c_{5}}{c_{1}} F_{a}(r-1, N-r-s+1)\right)$

$=P\left(F_{15}\left[c_{1}, c_{5}, \lambda_{1}, O\right]>\frac{1-P}{1+\rho\left(\frac{N}{r}-1\right)} F_{\alpha}(r-1, N-r-s+1)\right)(3.15)$

onde $\lambda_{1}=\frac{\tilde{\mu}^{\prime} P_{1} \tilde{\mu}}{2 C_{1}} \quad$ e deve ser fixado para se calcular tais probabilidades.

O mesmo ocorre com a hipótese $H_{0}: \beta_{j}=0$ vs $H_{1}: \beta_{j} \neq 0$ para algum $j=1,2, \ldots, s$, ou seja: P(erro tipo II) $=$

$=P\left(F_{25}\left[c_{2}, c_{5}, \lambda_{2}, 0\right]<\frac{1-P}{1+\rho\left(\frac{N}{S}-1\right)} F_{\alpha}(s-1, N-r-s+1)\right)(3.16)$ com $\quad \lambda_{2}=\frac{\tilde{\mu} \cdot P_{2} \tilde{\mu}}{2 c_{2}} \quad, 0$ qual deve ser fixado.

Essas probabilidades são dadas pela TABELA A.6. do apéndice, segundo os mesmos valores de $\rho, r, s, K$; nivel de significancia $0.05 e \lambda_{1} e \lambda_{2}$ com os valores 0 ; 5 ; 20. Observa-se que se $k=1$, não tem sentido o caso de equicorrel ação aqui estudado, ou seja não existe $p$, - que equivalente a tomar-se $p=0$ e $k=1$ e modelo se reduz a um modelo de 2 fatores fixos com estrutura de erros independentes (usual). 
b.2. Equicorrelação das observaçăes dentro de cada nivel de um dos fatores.

Neste caso considera-se uma equicorrelação dentro dos niveis de um dos fatores, digamos $\alpha$, ass $\pi$ ao invés de (3.12) tem-se:

$\operatorname{cov}\left(y_{i j k}, y_{i}, j, k,\right)=\left\{\begin{array}{lllll}\alpha^{2} & \text { se } & i=i, & j=j, \text { e } & k=k \\ \rho \sigma^{2} & \text { se } & i=i, & j \neq j & \text { ou } \\ 0 & \text { se } & i \neq i & \end{array}\right.$ Assim pode se escrever $\Omega=\frac{\mathrm{I}}{\mathrm{r}} \mathrm{V} \quad$ onde

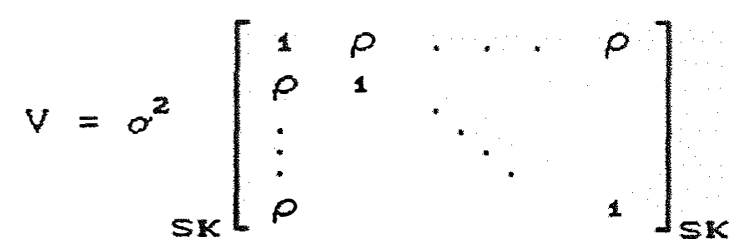

com $\Omega \in \mathscr{H}$, isto $6 \quad \Omega=c_{0} P_{0}+c_{1} P_{1}+c_{2} P_{2}+c_{5} P_{5}=$ $=\left(c_{0}-c_{5}\right) P_{0}+\left(c_{1}-c_{5}\right) P_{1}+\left(c_{2}-c_{5}\right) P_{2}+c_{5} I_{N} \quad$ onde $P_{0}, P_{1}, P_{2} e P_{5}$ são dadas por (3.8). Obtendo-se - seguinte sistema de equaçőes:

$$
\begin{array}{ll}
\left(c_{0}-c_{5}\right) \frac{1}{N}+\left(c_{1}-c_{5}\right) \frac{r-1}{N}+\left(c_{2}-c_{5}\right) \frac{s-1}{N}+c_{5} & =o^{2} \\
\left(c_{0}-c_{5}\right) \frac{1}{N}+\left(c_{1}-c_{5}\right) \frac{r-1}{N}+\left(c_{2}-c_{5}\right) \frac{s-1}{N} & =p \sigma^{2} \\
\left(c_{0}-c_{5}\right) \frac{1}{N}+\left(c_{1}-c_{5}\right) \frac{r-1}{N}+\left(c_{2}-c_{5}\right) \frac{-1}{N} & =0 \sigma^{2} \\
\left(c_{0}-c_{5}\right) \frac{1}{N}+\left(c_{1}-c_{5}\right) \frac{-1}{N}+\left(c_{2}-c_{5}\right) \frac{5-1}{N} & =0
\end{array}
$$


cuja sol ução é

$$
\begin{aligned}
& c_{0}=c_{1}=\sigma^{2}\left[1+\left(\frac{N}{r}-1\right) p\right] \\
& c_{2}=c_{5}=\sigma^{2}[1-\rho]
\end{aligned}
$$

onde

$$
\frac{-1}{\frac{N}{r}-1}<\rho<1
$$

Sob as restriçđes parametricas $\sum \alpha_{i}=\Sigma \beta_{j}=0$, obtem-se 0 quadro de análise de variância:

Quadro 3.9. Análise de variância para o modelo (3.7) caso b.2.

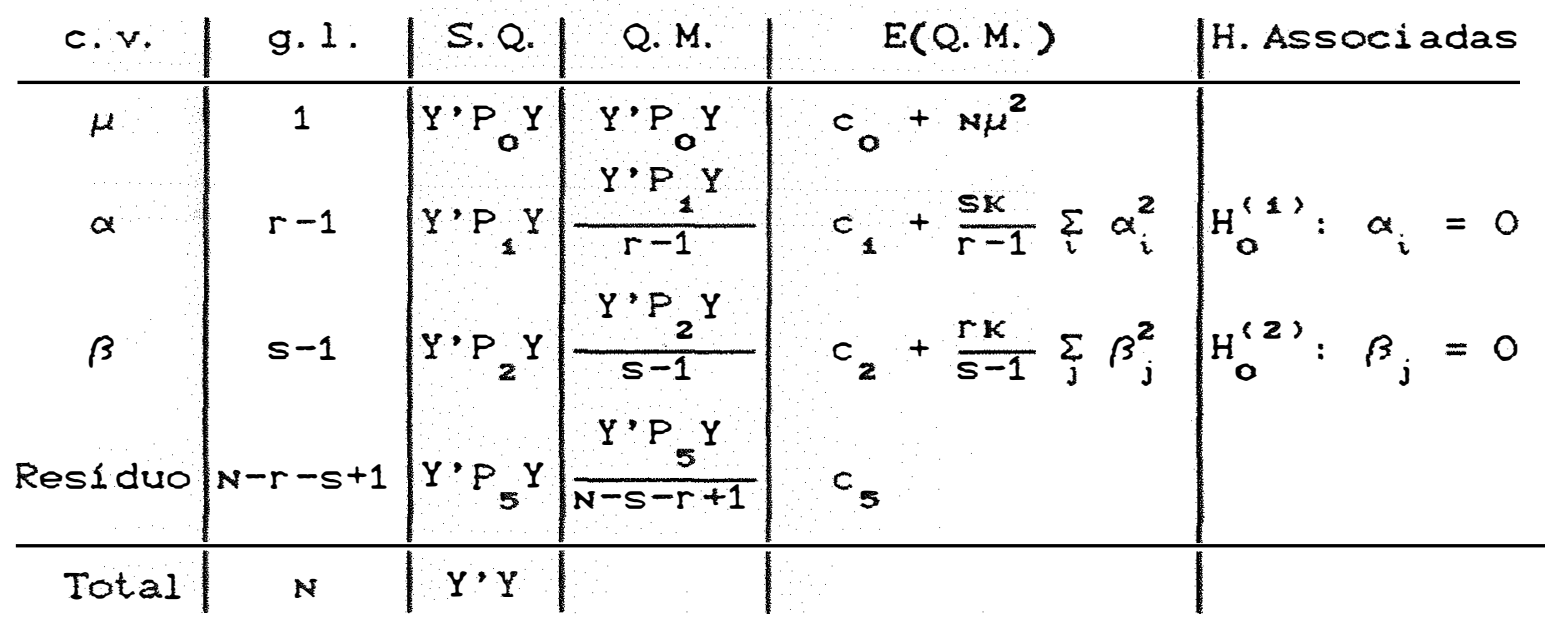

Assim a estatistica usual para o teste de $H_{0}: a_{i}=0$ vo $H_{1}: a_{i} \neq 0$ para algum $i=1,2, \ldots, r \quad 6$ dada por:

$$
\begin{aligned}
F_{15}\left(c_{1}, c_{5}\right) & =F_{15}\left(\sigma^{2}\left[1+\left(\frac{N}{r}-1\right) \rho\right], \sigma^{2}[1-\rho]\right)= \\
& =\frac{1-\rho}{1+\left(\frac{N}{r}-1\right) \rho} F\left[r-1, N-r-s+1, \lambda_{1}, 0\right],
\end{aligned}
$$

cujo comprometimento nas probalidades do erro tipo I é o mesmo dado por (3.13). Más quanto ao teste para a hipstese 
$H_{0}: \beta_{j}=0$ vo $H_{1}: \beta_{j} \neq 0$ para algum $j=1,2, \ldots, s$, tem-se a estatistica $F_{25}\left(c_{2}, c_{5}\right)=F_{25}(1,1)$, que $6 a$ estatistica usualmente empregada para esse teste, ou seja, as probabilidades de erro tipo I não sofrerão alteraçz̃es no seu nivel de significáncia, ao contrário do que ocorre em (3.14). Pode-se notar, por (3.14), que no caso de dependéncia somente dentro das caselas o nivel de significancia para essa mesma hipótese sofre alteraçōes.

Embora as probabilidades dos erros, para a hipótese $H^{(1)}$ sejam as mesmas dada em (3.13), apresentam-se novas tabelas dessas probabilidades, mudando alguns valores de "p". Para as probabilidades do erro tipo I, veja TABELA A.7. do apéndice. Quanto as probabilidades do erro tipo II, temos, sob as hipóteses alternativas, que as estatisticas $F_{15}\left(c_{1}, c_{5}\right)$ e $F_{25}\left(c_{2}, c_{5}\right)$ têm distribuiçres $F\left[r-1, N-r-s+1, \lambda_{1}, 0\right] \otimes F\left[s-1, N-r-s+1, \lambda_{2}, 0\right]$ respecti vamente, onde $\lambda_{1}=\frac{\tilde{\mu}^{3} P_{1} \tilde{\mu}}{2 c_{1}} \ominus \lambda_{2}=\frac{\tilde{\mu} P_{2} \tilde{\mu}}{2 c_{2}}$ com $\tilde{\mu}$ dado por (3.10). Para a hipótese $H_{0}: \alpha_{i}=0$ ve $H_{1}: \alpha_{i} \neq 0$ tem-se: P(erro tipo II) $=$ $=P\left(F_{15}\left[c_{1}, c_{5}, \lambda_{1}, 0\right]<\frac{c_{5}}{c_{1}} F_{\alpha}(r-1, N-r-s+1)\right)=$ $=P\left(F_{15}\left[c_{1}, c_{2}, \lambda_{1}, 0\right]<\frac{1-\rho}{1+(s k-1) \rho} F_{\alpha}(r-1, N-r-s+1)\right)$ Para a hipotese $H_{0}: \beta_{j}=0$ vs $H_{1}: \beta_{j} \neq 0$ para algum $j=1,2, \ldots, s$, tem-se 
P(erro tipo II $)=P\left(F_{25}\left[c_{2}, c_{5}, \lambda_{2}, 0\right]<F_{\alpha}(s-1, N-r-s+1)\right)$ que $e$ a probabilidade usual do erro tipo II, segundo 0 valor $\lambda_{2}$, pois $c_{2}=c_{5}$.

Logo pode-se ver que violaçres nas suposiçres de independéncia, neste caso, tambem afetam as probabilidades de erro tipo II para a hipotese $H_{0}: \alpha_{i}=0$ vo $H_{1}: \alpha_{i} \neq 0$ para algum $i$, e não afetam para a hipótese $H_{0}: \beta_{j}=0$ vs $H_{i}: \beta_{j} \neq 0$ para algum $j$. A TABELA A. 8. do apendice fornece essas probabilidades para diversos valores de $r, s, K, \rho, \lambda$ e nivel nominal de significancia de 0,05 .

\subsubsection{MODELOS COM INTERAÇÃo}

\section{a. Erros independentes}

Considera-se 0 modelo (3.7) mais a interação entre os fatores principais, isto 6

$$
\begin{aligned}
& y_{i j k}=\mu+\alpha_{i}+\beta_{j}+\alpha \beta_{i j}+e_{i j k} \quad \text { onde } i=1,2, \ldots, r \text {, } \\
& j=1,2, \ldots, s \text { e } k=1,2, \ldots, k, \text { com } N=r s k \text {. } \\
& \text { As somas de quadrados devida aos fatores são: } \\
& S Q(\mu)=Y \cdot P_{O} Y \quad S Q(\alpha \beta) \quad=Y^{\prime} P_{3} Y \\
& S Q(\alpha)=Y \cdot P_{1} Y \quad \text { SORes } 1 \text { duo }=Y \cdot P_{5} Y \\
& \operatorname{SQ}(\beta)=Y^{\prime} P_{Z} Y \quad \text { SOTotal }=Y^{\prime} I_{N} Y
\end{aligned}
$$




$$
\begin{aligned}
& P_{3}=I_{r s} \otimes K_{K}-K_{N}-P_{1}-P_{2}=I_{r s} \otimes K_{K}-I_{r} \otimes K_{s r}-K_{r} \otimes I_{s} \otimes K_{K}+K_{N} \\
& P_{5}=I_{N}-\left(P_{0}+P_{1}+P_{2}+P_{3}\right) \\
& \text { com } \quad r\left(P_{3}\right)=r s-r-s+1=(r-1)(s-1) \\
& \quad r\left(P_{5}\right)=N-r s=r s(K-1)
\end{aligned}
$$

Os projetores ortogonais de $Y_{N}$ em algum subespaço de $C(X)$ são as matrizes $P_{i}, \quad i=0,1,2,3,5$ simetricas e idempotentes.

o vetor $Y_{N}$ de observaçoes é tal que $Y_{N} \cap N(\tilde{\mu}, \Omega)$ onde $\tilde{\mu}$ e dado por $(3.10)$ e $\Omega$ e tal que $\Omega \in \mathcal{Z}=\left\{\Omega: \Omega=\sum_{i} c_{i} P_{i} \operatorname{com} c_{i}>0, p^{\prime} i=0,1,2,3,5\right\}$ e como $\Omega=\sigma^{2} I_{N}$ então $c_{i}=\sigma^{2}$ para todo $i$.

Considerando-se as restriçöes parametricas dadas por $\sum_{i} \alpha_{i}=\sum_{j} \beta_{j}=\sum_{i} \alpha \beta_{i j}=\sum_{j} \alpha \beta_{i j}=0$ e ainda (3.4) e (3.9), tem-se o seguinte quadro de análise

\begin{tabular}{|c|c|c|c|c|}
\hline c. V. & g. 1 . & S. Q. & $E(Q . M)$. & H. Associadas \\
\hline$\mu$ & 1 & $Y \cdot P_{O} Y$ & $\alpha^{2}+N \mu^{2}$ & \\
\hline$\alpha$ & $r-1$ & $Y \cdot P_{1} Y$ & $\alpha^{2}+\frac{s k}{r-1} \sum_{i} \alpha_{i}^{2}$ & $H_{0}^{(1)}: \alpha_{i}=0$ \\
\hline$\beta$ & $s-1$ & $Y^{\prime} P_{2} Y$ & $\alpha^{2}+\frac{r k}{s-1} \sum_{j} \beta_{j}^{2}$ & $H_{0}^{(2)}: \beta_{j}=0$ \\
\hline$\alpha \beta$ & $(r-1)(s-1)$ & $Y \cdot P_{a} Y$ & $\alpha^{2}+\frac{k}{(r-1)(s-1)} \sum_{i j} \alpha \beta_{i j}^{2}$ & $\mathrm{H}_{0}^{(3)}:(\alpha \beta)_{i j}=0$ \\
\hline Res & $\operatorname{rs}(x-1)$ & $Y^{\prime} P_{5} Y$ & $a^{2}$ & \\
\hline Tot & $\mathrm{N}$ & $Y \cdot Y$ & & \\
\hline
\end{tabular}
de variancia:

Quadro 3.10. Análise de variancia para o modelo (3.7) com interação, caso a.. 
e os testes para as hipóteses principais são todos feitos contra o residuo e mantém o nivel de significáncia desejado, como era de se esperar, na análise de variancia usual para esse modelo.

b. Erros não independentes

Serão tratados alguns casos de violaçzes, cujos efeitos possam ser estudados segundo a metodologia aqui empregada, e que sejam mais usuais.

\section{b. 1. Equicorrelação das observações somente dentro da mesma casela}

Neste caso a covariancia entre duas observaçós na mesma casela é dada por:

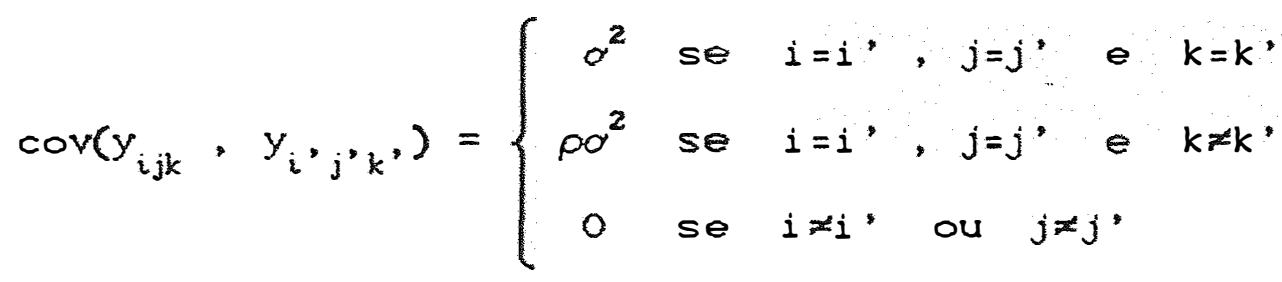

As somas de quadrados $Y$ 'P $P_{i} Y P^{\prime} \quad i=0,1,2,3,5$ são as mesmas dadas por $(3.17)$ e a matriz $\Omega$ dada por $\Omega=I_{\text {rs }} \otimes V \quad$ onde 


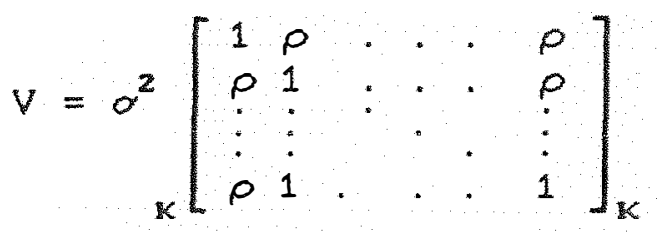

Como $\Omega \in \mathcal{H}=\left\{\Omega: \Omega=\sum_{i} c_{i} P_{i}, c_{i}>0 p / i=0,1,2,3,5\right\}$, resolve-se o seguinte sitema de equaçôes:

$$
\begin{array}{ll}
\left(c_{0}-c_{5}\right) \frac{1}{N}+\left(c_{1}-c_{5}\right) \frac{r-1}{N}+\left(c_{2}-c_{5}\right) \frac{s-1}{N}+\left(c_{3}-c_{5}\right) \frac{r s-s-r+1}{N}+c_{5}=0^{2} & =00^{2} \\
\left(c_{0}-c_{5}\right) \frac{1}{N}+\left(c_{1}-c_{5}\right) \frac{r-1}{N}+\left(c_{2}-c_{5}\right) \frac{s-1}{N}+\left(c_{3}-c_{5}\right) \frac{r s-s-r+1}{N} & =0 \\
\left(c_{0}-c_{5}\right) \frac{1}{N}+\left(c_{1}-c_{5}\right) \frac{r-1}{N}+\left(c_{2}-c_{5}\right) \frac{-1}{N}+\left(c_{3}-c_{5}\right) \frac{1-r}{N} & =0 \\
\left(c_{0}-c_{5}\right) \frac{1}{N}+\left(c_{1}-c_{5}\right) \frac{-1}{N}+\left(c_{2}-c_{5}\right) \frac{S-1}{N}+\left(c_{3}-c_{5}\right) \frac{1-s}{N} & =0 \\
\left(c_{0}-c_{5}\right) \frac{1}{N}+\left(c_{1}-c_{5}\right) \frac{-1}{N}+\left(c_{2}-c_{5}\right) \frac{-1}{N}+\left(c_{5}-c_{5}\right) \frac{1}{N} & =0
\end{array}
$$

obtendo-se a seguinte solução:

$$
\begin{aligned}
c_{0}=c_{1}=c_{2}= & c_{3}=\sigma^{2}\left[\left(\frac{N}{r s}-1\right) \rho+1\right]=\sigma^{2}[(k-1) \rho+1] \\
c_{5} & =\sigma^{2}[1-\rho]
\end{aligned}
$$

para $-\left(\frac{N}{r S}-1\right)^{-1}<\rho<1 \quad$ ou $\frac{-1}{K-1}<\rho<1$

Considerando as restriçర̃es dadas por (3.18) e por (3.4) e (3.9), tem-se o quadro de análise de variancia: 
Quadro 3.11. Análise de variancia para o modelo (3.7) com interação, caso b. 1.

\begin{tabular}{|c|c|c|c|c|}
\hline c.v. & 9.1 & S. $Q$. & $E(Q . M)$. & H. Associ adas \\
\hline$\mu$ & 1 & $Y \cdot P_{0}^{Y}$ & $c_{0}+N \mu^{2}$ & \\
\hline$\alpha$ & $r-1$ & $Y \cdot P Y$ & $c_{1}+\frac{s k}{r-1} \sum_{i} a_{i}^{2}$ & $H_{0}^{(1)}: \alpha_{i}=0$ \\
\hline$\beta$ & $s-1$ & $Y \cdot P_{2} Y$ & $c_{2}+\frac{r k}{s-1} \sum_{j} \beta_{j}^{2}$ & $H_{0}^{(2)}: \beta_{j}=0$ \\
\hline$\alpha \beta$ & $(r-1)(s-1)$ & $Y \cdot P_{3} Y$ & $c_{3}+\frac{k}{(r-1)(s-1)} \sum_{i j} \alpha \beta_{i j}^{2}$ & $\mathrm{H}_{\mathrm{O}}^{(a)}:(\alpha \beta)_{i j}=0$ \\
\hline Res & $r s(k-1)$ & $Y P^{\prime} Y$ & $c_{5}$ & \\
\hline Total & $\mathbf{N}$ & $Y: Y$ & & \\
\hline
\end{tabular}

Os testes usuais são feitos em relação ao resíduo, - que neste caso de violação comprometerá as probabilidades de erro tipo I e II para qualquer uma das hipóteses principais. O fator de proporcionalidade das distribuiç̧̋es (3.13), (3.14) são iguais para as trés hipóteses principais. Teremos, para qualquer uma das hipoteses principais, que: $P($ errotipo I $)=$ $=P\left(F_{i 5}\left(c_{i}, c_{5}\right) \geq \frac{1-p}{1+\rho \alpha k-15} F_{\alpha}\left(r\left(P_{i}\right), r s(k-1)\right)\right) \geqslant a$ para $i=1,2,3$, ou seja P(erro tipo I) $\left\{\begin{array}{rrr}>a & \text { se } & 0<p<1 \\ =\alpha & \text { se } & p=0 \\ <\alpha & \text { se } \frac{-1}{k-1}<p<0\end{array}\right.$ onde $\alpha$ o nivel de significancia supostamente correto usado pelo pesquisador. A TABELA A. 8 . do apéndice pornece as probabilidades dos erros tipo I para $H^{(1)}, H^{(2)}$ e $H^{(3)}$, 
segundo valores de $\rho, r, k$ e nivel nominal de significancia $\alpha=0,05$.

Quanto as probabilidades do erro tipo II,

tem-se P(erro tipo II $)=$

$=P\left(F_{i 5}\left[c_{i}, c_{5}, \lambda_{i}, 0\right]<\frac{1-p}{1+\rho(k-1)} F_{\alpha}\left(r\left(P_{i}\right), \operatorname{rs}(k-1)\right)\right)$

onde $\lambda_{i}=\frac{\tilde{\mu} P_{i} \tilde{\mu}}{2 c_{i}}$ para $i=1,2, e 3$ com $\tilde{\mu}$, dado por (3.10) para $\mu_{i j}=\mu+\alpha_{i}+\beta_{j}+\alpha \beta_{i j} \theta$

$$
\begin{aligned}
& c_{i}=\sigma^{2}[1+(k-1) \rho] \\
& c_{5}=\sigma^{2}[1-\rho]
\end{aligned}
$$

Essas probabilidades são dadas no apéndice pela TABELA A.10. para os mesmos valores de $\rho, r, s, k$ e $\alpha$ da TABELA A. 9 e $\lambda_{i}=0,5$ e 20 para $i=1,2, e 3$.

b.2. Equicorrelação das observaçżes dentro dos niveis de un dos fatores, digamos $\alpha$

Neste caso a covariancia entre duas observaçöes dentro do mesmo nível do fator $\alpha$ dada por:

$\operatorname{cov}\left(y_{i j k}, y_{i}^{\prime} j^{\prime} k^{\prime}\right)=\left\{\begin{array}{cccc}\alpha^{2} & \text { se } i=i, j=j, & \text { e } & k=k^{\prime} \\ \rho \alpha^{2} & \text { se } i=i, j \neq j, & \text { ou } k \neq k \\ 0 & \text { se } i \neq i & \end{array}\right.$ 
As somas de quadrados $Y$ ' $P_{i} Y$ para $i=0,1,2,3,5$ săo as mesmas dadas por (3.17) e a matriz $\Omega$ de variancias e covariáncias é dada por $\Omega=I_{r} \otimes$ com

$$
V=\sigma_{S K}^{2}\left[\begin{array}{lllll}
1 & \rho & \cdots & \cdot & \rho \\
\rho & 1 & \cdots & \cdot & \rho \\
\vdots & & \cdot & \vdots \\
\rho & \cdots & \cdot & 1
\end{array}\right]_{S K}
$$

Como $\Omega \in \mathcal{Z}$, tem-se o seguinte sistema de equaços:

$$
\begin{aligned}
& \left(c_{0}-c_{5}\right) \frac{1}{N}+\left(c_{1}-c_{5}\right) \frac{r-1}{N}+\left(c_{2}-c_{5}\right) \frac{s-1}{N}+\left(c_{3}-c_{5}\right) \frac{r s-r-s+1}{N}+c_{5}=\alpha^{2} \\
& \left(c_{0}-c_{5}\right) \frac{1}{N}+\left(c_{1}-c_{5}\right) \frac{r-1}{N}+\left(c_{2}-c_{5}\right) \frac{s-1}{N}+\left(c_{3}-c_{5}\right) \frac{r s-r-s+1}{N}=p \sigma^{2} \\
& \left(c_{0}-c_{5}\right) \frac{1}{N}+\left(c_{1}-c_{5}\right) \frac{r-1}{N}+\left(c_{2}-c_{5}\right) \frac{-1}{N}+\left(c_{3}-c_{5}\right) \frac{1-r}{N}=0 \\
& \left(c_{0}-c_{5}\right) \frac{1}{N}+\left(c_{1}-c_{5}\right) \frac{-1}{N}+\left(c_{2}-c_{5}\right) \frac{5-1}{N}+\left(c_{3}-c_{5}\right) \frac{1-s}{N}=0 \\
& \left(c_{0}-c_{5}\right) \frac{1}{N}+\left(c_{1}-c_{5}\right) \frac{-1}{N}+\left(c_{2}-c_{5}\right) \frac{-1}{N}+\left(c_{3}-c_{5}\right) \frac{1}{N}=0
\end{aligned}
$$

obtendo-se a sol ucăo:

$$
\begin{aligned}
& c_{0}=c_{1}=\sigma^{2}\left[\left(\frac{N}{r}-1\right) \rho+1\right]=\sigma^{2}[(s k-1) \rho+1] \\
& c_{2}=c_{3}=c_{5}=\sigma^{2}[1-\rho]
\end{aligned}
$$

para $-\left(\frac{N}{r}-1\right)^{-1}<\rho<1$ ou seja $\frac{-1}{s r-1}<\rho<1$

Considerando-se as restriçes dadas por (3.18) e ainda por (3.4) e (3.9) tem-se o quadro de análise de variancia: 
Quadro 3.12. Análise de variancia para o modelo (3.7) com interação, caso b. 2 .

\begin{tabular}{|c|c|c|c|c|}
\hline$c . v$. & 9.1 & S. Q. & $E(Q . M)$. & H. Associ adas \\
\hline$\mu$ & 1 & $Y \cdot P_{0} Y$ & $c_{0}+N \mu^{2}$ & \\
\hline$\alpha$ & $r-1$ & $Y^{\prime} P_{1} Y$ & $c_{1}+\frac{s k}{r-1} \sum_{i} \alpha_{i}^{2}$ & $H_{0}^{(1)}: \alpha_{i}=0$ \\
\hline$\beta$ & $s-1$ & $Y P_{2} Y$ & $c_{2}+\frac{r k}{s-1} \sum_{j} \beta_{j}^{2}$ & $H_{0}^{(z)}: \beta_{j}=0$ \\
\hline$\alpha \beta$ & $(r-1)(s-1)$ & $Y P_{3} Y$ & $c_{a}+\frac{k}{(r-1)(s-1)} \sum_{i j} \alpha \beta_{i j}^{2}$ & $H_{0}^{(3)}:(\alpha \beta)_{i j}=0$ \\
\hline Res & $\operatorname{rs}(x-1)$ & $Y P_{5} Y$ & $c_{5}$ & \\
\hline al & $\mathbf{N}$ & $Y \cdot Y$ & & \\
\hline
\end{tabular}

Para as hipoteses principais $H_{0}: \beta_{j}=0$ vs $H_{1}: \beta_{j} \neq 0$ para algum $j=1,2, \ldots, s$ e $H_{0}: \alpha \beta_{i j}=0$ vs $H_{1}: a \beta_{i j} \neq 0$ para algum par $(i, j) \operatorname{com} i=1,2, \ldots, r$ e $j=1,2, \ldots, s$, o experimentador poderá utilizar os testes usuais sem comprometer as probabilidades do erros tipos I e II enquanto que para a hipótese $H_{0}: \alpha_{i}=0 v s H_{1}: \alpha_{i} \neq 0$ para algum $i=1,2, \ldots, r, 0$ comprometimento acontece como ocorreu em 3.3.2-b.1., isto 6:

$P($ erro tipo $I)=P\left[F_{15}\left(c_{1}, c_{5}\right) \geq \frac{1-\rho}{1+(s k-1) \rho} F_{\alpha}(r-1, r s(k-1))\right] \gtreqless \alpha$ ou seja P(errotipo I) $\left\{\begin{array}{ccc}>a & \text { se } & 0<\rho<1 \\ =\alpha & \text { se } & \rho=0 \\ <a & \text { se } \frac{-1}{k s-1}<\rho<0\end{array}\right.$

Essas probabilidades são dadas pela TABELA A.11. do apéndice, segundo valores de $\rho, r, s, k$ e $\alpha=0.05$ Quanto ao erro tipo II tem-se: 
P(erro tipo II $)=$

$=P\left(F_{15}\left[c_{t}, c_{s}, \lambda_{1}, 0\right]<\frac{1-P}{1+C \approx K-1) \rho} F_{\alpha}(r-1, r s(k-1))\right)$

onde $\quad \lambda_{1}=\frac{\tilde{\mu} P_{1} \tilde{\mu}}{2 c_{1}}$, com $\tilde{\mu}$ dado por $(3.10)$ para $\mu_{i j}=\mu+\alpha_{i}+\beta_{i}+\alpha \beta_{i j}$. Para essas probabilidades veja TABELA A.12. , segundo os mesmos valores de $\rho, r, s, k$ a $\operatorname{com} \lambda_{1}=0,5$ e 20

b. 3. Equicorrelacão das observacões dentro das caselas por " $\rho_{1}$ ", e dentro do mesmo nível de um dos fatores, mas em caselas diferentes por " $P_{2} "$

Então a covariancia entre duas observaçōes quaisquer é dada por

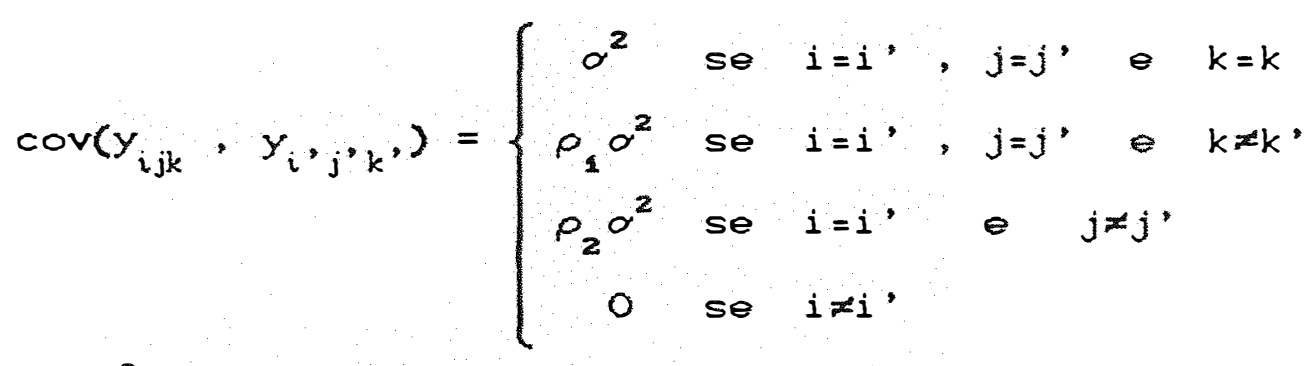

$$
\begin{aligned}
& \text { e } \Omega=\sigma^{2} I_{r} \otimes\left[I_{S} \otimes V_{K}+V_{K S}\right] \text { onde } \\
& V_{K}=\left[\begin{array}{cccc}
1-\rho_{2} & \rho_{1}-\rho_{2} & \cdot & \rho_{1}-P_{2} \\
\rho_{1}-\rho_{2} & 1-\rho_{2} & \ddots & \rho_{1}-\rho_{2} \\
\rho_{1}-\rho_{2} & \rho_{1}-\rho_{2} & \cdot & \cdot \\
& & 1-\rho_{2}
\end{array}\right]_{K}
\end{aligned}
$$




$$
V_{K S}=s k p_{2} K_{s k}=\rho_{2}\left[\begin{array}{cccccc}
1 & 1 & \cdots & \cdot & \cdot & 1 \\
1 & 1 & \vdots & \cdot & \cdot & 1 \\
\vdots & \vdots & \ddots & \cdot & \vdots \\
1 & 1 & \cdot & \vdots & \vdots & 1
\end{array}\right]_{s K}
$$

sendo o vetor de observações $Y$ dado por (3.8).

Como $\Omega \in \mathscr{F}$ tem-se o seguinte sistema de equações, nas constantes $c_{i}$ para $i=0,1,2,3$ e 5

$$
\begin{array}{ll}
\left(c_{0}-c_{5}\right) \frac{1}{N}+\left(c_{1}-c_{5}\right) \frac{r-1}{N}+\left(c_{2}-c_{5}\right) \frac{s-1}{N}+\left(c_{3}-c_{5}\right) \frac{r 5-r-s+1}{N}+c_{5}=\sigma^{2} \\
\left(c_{0}-c_{5}\right) \frac{1}{N}+\left(c_{1}-c_{5}\right) \frac{r-1}{N}+\left(c_{2}-c_{5}\right) \frac{5-1}{N}+\left(c_{3}-c_{5}\right) \frac{r 5-r-s+1}{N} & =p_{1} \sigma^{2} \\
\left(c_{0}-c_{5}\right) \frac{1}{N}+\left(c_{1}-c_{5}\right) \frac{r-1}{N}+\left(c_{2}-c_{5}\right) \frac{-1}{N}+\left(c_{3}-c_{5}\right) \frac{1-r}{N} & =p_{2} \alpha^{2} \\
\left(c_{0}-c_{5}\right) \frac{1}{N}+\left(c_{1}-c_{5}\right) \frac{-1}{N}+\left(c_{2}-c_{5}\right) \frac{5-1}{N}+\left(c_{3}-c_{5}\right) \frac{1-s}{N} & =0 \\
\left(c_{0}-c_{5}\right) \frac{1}{N}+\left(c_{1}-c_{5}\right) \frac{-1}{N}+\left(c_{2}-c_{5}\right) \frac{-1}{N}+\left(c_{3}-c_{5}\right) \frac{1}{N} & =0
\end{array}
$$

cuja sol ução e:

$$
\begin{aligned}
c_{0}=c_{1} & =\sigma^{2}\left[1+\rho_{1}(k-1)+p_{2} k(s-1)\right] \\
c_{2}=c_{3} & =\sigma^{2}\left[1+\rho_{1}(k-1)-\rho_{2} k\right] \\
c_{5} & =\sigma^{2}\left[1-\rho_{1}\right]
\end{aligned}
$$

e como $c_{i}>0$ para $i=0,1,2,3$ e 5 tem-se

$1+p_{1}(k-1)+p_{2} k(s-1)>0$ e $1+p_{1}(k-1)-p_{2} k>0$,

resultando:

$$
\begin{aligned}
& \text { se } p_{2}>0 \Longrightarrow \rho_{1}>\frac{-1+\rho_{2} k}{k-1} \\
& \text { se } p_{2}=0 \Longrightarrow p_{1}>\frac{-1}{k-1} \\
& \text { se } p_{2}<0 \quad \longrightarrow \quad p_{1}>\frac{-1-p_{2} k(s-1)}{k-1}
\end{aligned}
$$

com $\rho_{1} e P_{2}$ no intervalo $[-1,1]$ 
- quadro de análise de variáncia, considerando-se as restriçōes dadas por (3.18) e ainda por (3.4) e (3.9), é dado por:

Quadro 3.14. Análise de variancia para o modelo (3.7) com interação, caso b. 3.

\begin{tabular}{|c|c|c|c|c|}
\hline c.V. & g. 1 . & S. Q. & $E(Q . M)$. & H. Associadas \\
\hline$\mu$ & 1 & $Y \cdot P_{0} Y$ & $c_{0}+N \mu^{2}$ & \\
\hline$\alpha$ & $r-1$ & $Y P_{1} Y$ & $c_{0}+\frac{s k}{r-1} \sum_{i} \alpha_{i}^{2}$ & $H_{0}^{(1)}: a_{i}=0$ \\
\hline$\beta$ & $s-1$ & $Y P_{2} Y$ & $c_{2}+\frac{r k}{s-1} \sum_{j} \beta_{j}^{2}$ & $H_{0}^{(2)}: \beta_{j}=0$ \\
\hline$\alpha \beta$ & $(r-1)(s-1)$ & $Y \cdot P_{3} Y$ & $c_{2}+\frac{k}{(r-1)(s-1)} \sum_{i j} a \beta_{i j}^{2}$ & $H_{0}^{(3)}:(\alpha \beta)_{i j}=0$ \\
\hline Res & $r s(k-1)$ & $Y \cdot P_{5} Y$ & $c_{5}$ & \\
\hline Tot & $N$ & $Y^{\prime} Y$ & & \\
\hline
\end{tabular}

Utilizando-se os testes usuais, para as hipóteses principais, haverá, para todas elas, o comprometimento das probabilidades dos erros tipos I e II. Pode-se notar que nenhum dos testes $H_{0}^{(1)}: \alpha_{i}=0$, $H_{0}^{(2)}: \beta_{j}=0$ ou $H_{0}^{(3)}: \alpha \beta_{i j}=0$ pode ser feito com a exatidão do nível de significancia, pelos métodos usuais, mas se $P_{1}=P_{2}=\rho$ então tem-se

$$
\begin{gathered}
c_{0}=c_{1}=\alpha^{2}[1+\rho(s k-1)] \\
c_{2}=c_{3}=c_{5}=\sigma^{2}[1-\rho], \text { reduzindo-se ao caso }
\end{gathered}
$$

3.3.2.-b.2. anteriormente estudado, com testes exatos para $H_{0}^{(2)}: \beta_{j}=0$ e para $H_{0}^{(3)}: \alpha \beta_{i j}=0$.

$$
\text { Se } \rho_{2}=0 \text { tem-se } c_{0}=c_{1}=c_{2}=c_{3}=
$$

$=\sigma^{2}\left[1+\rho_{1}(k-1)\right] e c_{5}=\sigma^{2}\left[1-\rho_{1}\right]$ reduzindo-se ao caso 3.3.2.-b.1. já estudado anteriormente sem testes 
usuais exatos para as hipóteses principais.

Se $s=1$, năo se tem modelo com doís fatores e sim com um único fator, logo $c_{2} e$ ca nå existem, e $c_{0}=c_{1}=\alpha^{2}\left[1+p_{1}(k-1)\right] \quad \theta \quad c_{5}=\alpha^{2}\left[1-p_{1}\right]$, reduzindo-se ao caso de um fator fixo e balanceado, estudado pelos autores citados no item 2.2., do capitulo 2 .

Então pelas observaçōes anteriores, pode-se ver que este caso é mais geral do que aqueles planejamentos de efeitos fixos e balanceados, já estudados.

Quanto ao erro tipo I tem-se P(erro tipo I) = $=P\left(F_{i 5}\left(c_{i}, c_{5}\right) \geq \frac{c_{5}}{c_{i}} F_{\alpha}\left(r\left(P_{i}\right), r s(k-1)\right) \geqslant \notin \alpha\right.$

onde $F_{i 5}\left(c_{i}, c_{5}\right) \Leftrightarrow$ a estatistica usual para se testar a hipotese $H_{0}^{(i)}$ para $i=1,2$ e 3 .

Entå para a $H_{0}^{(1)}$, (considerando (3.19)), obtem-se

$$
P(e r r o t i p o I)\left\{\begin{array}{l}
>\alpha \text { se } p_{1}+(s-1) p_{2}>0 \\
=\alpha \text { se } p_{1}+(s-1) p_{2}=0 \\
<\alpha \text { se } p_{1}+(s-1) p_{2}<0
\end{array}\right.
$$

enquanto que para as hipóteses $H_{0}^{(2)} \odot H_{0}^{(3)}$, tem-se ( levando-se em conta ( 3.19 ) $)$ que:

P(errotipo I) $\left\{\begin{array}{l}>\alpha \text { se } P_{2}<P_{1} \\ =\alpha \text { se } P_{1}=P_{2} \\ <a \text { se } P_{2}>P_{1}\end{array}\right.$

Essas probabilidades são dadas pela TABELA A.13. do apendice. 
Quanto as probabilidades do erro tipo II.

obtem-se:

P(errotipo II $)=$

$=P\left(F_{i 5}\left[c_{i}, c_{5}, \lambda, 0\right]<\frac{c_{5}}{c_{i}} F_{\alpha}\left(r\left(P_{i}\right), r s(k-1)\right)\right)$

para $i=1.2$ e $3, \operatorname{com} \lambda_{i}=\frac{\tilde{\mu}_{i} P_{i}}{C_{i}} \quad \operatorname{com} \tilde{\mu}$

dado por $(3.10)$ - $\mu_{i j}=\mu+\alpha_{i}+\beta_{j}+\alpha \beta_{i j}$ Essas probabilidades são dadas pela TABELA A.14. do apêndice.

As tabelas são produzidas para alguns valores de $P_{2}$ isto é $P_{2}=0.1 ; 0.3$ e 0.5 para as probabilidades do erro tipo I e $P_{2}=0.3$ para as probabilidades do erro tipo II: $\operatorname{com} \lambda_{i}=0 ; 5$ e 20 para $i=1,2$, e 3 .

\subsubsection{PROCEDIMENTOS Alternativos}

Apresentam-se aqui sugestöes para efetuar-se estes testes sem o comprometimento das probabilidades dos erros tipos I e II. através de uma modificação do planejamento, considerando a realizaçăo de repetiçơes independentes de mesmo tamanho, com a inclusão de mais um fator (repetiçăo) no planejamento. 
a. Procedimento alternativo para o caso

3.3.1.b.2., cujo modelo é dado por (3.7)

Consideram-se agora b repetiçరes independentes entre si e também com relaça aos erros do modelo, de mesmo tamanho. Então o modelo passa a ser dado por:

$y_{i j l k}=\mu+\alpha_{i}+\beta_{j}+R_{L(i)}+e_{i j l k}$, onde $R_{L i}$ é o efeito da l-ésima repetiçăo dentro do i-ésimo nível do fator $a$, para $i=1,2, \ldots, r, j=1,2, \ldots, s, 1=1,2, \ldots, b$ e $\quad k=1,2 \ldots . ., k$. Considerando-se que $R_{l(i)} e e_{i j l k}$ são variáveis aleatórias independentes com distribuiçoes normais de média nula e matrizes de variancias e covariancias dada por $\Omega_{1}=\sigma_{R}^{2} I_{N}$ e $\Omega_{2}$ respectivamente, com $N=$ rsbk.

$$
\text { Tem-se } E\left(y_{i j l k}\right)=\mu+\alpha_{i}+\beta_{j}=\mu_{i j} \quad e
$$

$Y \cap N\left(\tilde{\mu} ; \Omega=V\left(\Omega_{1} \cdot \Omega_{2}\right)\right)$ onde o vetor de observaçðes Y é dado por:

$Y=\left[Y_{111}^{\prime} Y_{112}^{\prime} \ldots Y_{116}^{\prime} Y_{121}^{\prime} Y_{122}^{\prime} \ldots Y_{126}^{\prime} \ldots Y_{181}^{\prime} Y_{182}^{\prime} \ldots Y_{186}^{\prime} \cdots\right.$

$$
\left.\ldots Y_{211}^{\prime} Y_{212}^{\prime} \cdots Y_{216}^{\prime} \cdots Y_{r=1}^{\prime} Y_{r=2}^{\prime} \cdots Y_{r=b}^{\prime}\right]^{\prime}
$$

- $Y_{i j l}=\left(Y_{i j l 1}, y_{i j l z}, \ldots, y_{i j l k}\right)$

tem-se que $\quad \operatorname{cov}\left(y_{i j l k} \cdot y_{i \cdot j / k_{k}}\right)=$

$$
\begin{aligned}
& =E\left[\left(y_{i j l k}-E\left(y_{i j l k}\right)\right)\left(y_{i j l k}-E\left(y_{i \cdot j \cdot l \cdot k}\right)\right)\right]= \\
& =E\left[\left(R_{l i \ell}+e_{i j l k}\right)\left(R_{L \cdot(i \cdot)}+e_{i \cdot j \cdot l \cdot k}\right)\right]=
\end{aligned}
$$




$$
\begin{aligned}
& =E\left(R_{l(i)} R_{l \cdot(i \cdot)}\right)+E\left(\theta_{i j l k} \theta_{i \cdot j \cdot l \cdot k \cdot}\right)=
\end{aligned}
$$

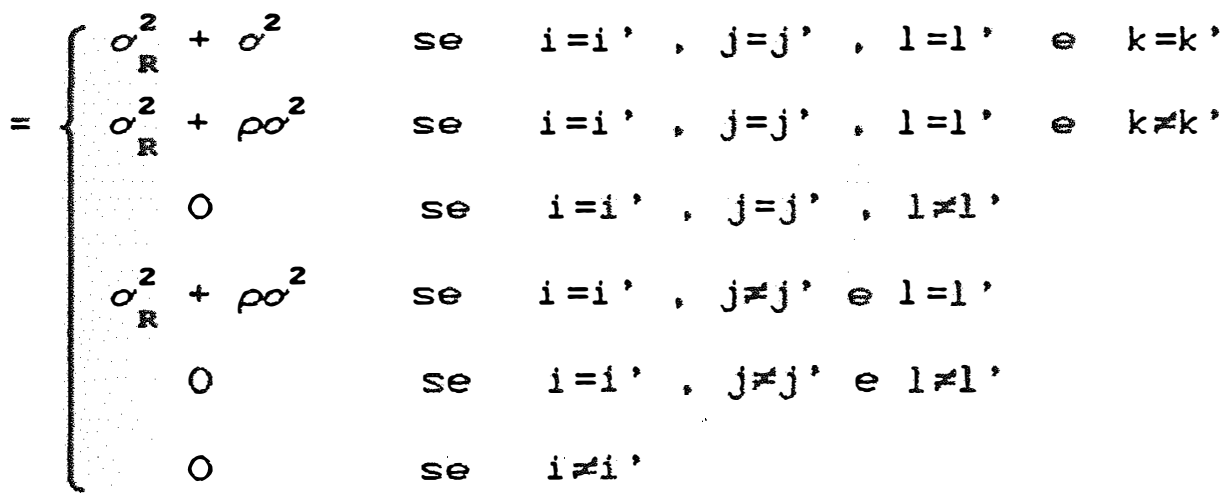

ou ainda pode-se escrever:

$$
\begin{aligned}
& \left.\Omega_{2}=\sigma^{2} f \rho\left[I_{r} \otimes s K_{S} \otimes I_{b} \otimes K_{K}\right]+(1-\rho) I_{N}\right\} \\
& \Omega_{1}=\sigma_{R}^{2} I_{N}, c o m \\
& V\left(\Omega_{1}, \Omega_{2}\right)=\Omega=\left(\sigma^{2} \rho+\sigma_{R}^{2}\right)\left[I_{r} \otimes s K_{S} \otimes I_{b} \otimes K K_{K}+\sigma^{2}(1-\rho) I_{N}\right]
\end{aligned}
$$

As somas de quadrados usuais para esse modelo são dadas por :

$$
\begin{aligned}
& \operatorname{SQ}(\mu)=Y^{\prime} P_{O} Y=\frac{1}{N} y^{2} \ldots \\
& S Q(\alpha)=Y \cdot P_{1} Y=\frac{1}{S b_{K}} \sum_{i} y_{i}^{2} \ldots-\frac{1}{N} y^{2} \ldots . \\
& S Q(\beta)=Y \cdot P_{2} Y=\frac{1}{\Gamma b_{K}} \sum_{j} y^{2} \cdot j \ldots-\frac{1}{N} y^{2} \ldots \\
& S Q(R)=Y \cdot P_{3} Y=\frac{1}{S K} \sum_{i l} Y_{i, l}^{2} .-\frac{r}{N} \sum_{i} Y_{i}^{2} \ldots \\
& \text { So(Residuo) } \quad=\quad Y \cdot P_{5} Y
\end{aligned}
$$

$$
\begin{aligned}
& \operatorname{com} P_{0}=K_{N} \quad \operatorname{com} \quad r\left(P_{0}\right)=1 \\
& P_{1}=I_{r} \otimes K_{s b K}-K_{N} \quad \text { com } r\left(P_{1}\right)=r-1 \\
& P_{2}=K_{r} \otimes I_{s} \otimes K_{b K}-K_{N} \quad \operatorname{com} r\left(P_{2}\right)=s-1 \\
& P_{a}=I_{r} \otimes K_{s} \otimes I_{b} \otimes K_{K}-I_{r} \otimes K_{s b K} \operatorname{com} r\left(P_{a}\right)=r(b-1) \\
& P_{*}=I_{r} \otimes K_{S} \otimes I_{b} \otimes K_{K} \quad \text { com } r\left(P_{*}\right)=r b \\
& P_{5}=I_{N}-\left(P_{0}+P_{1}+P_{2}+P_{3}\right)= \\
& =I_{N}-P_{-}-P_{2} \quad \operatorname{com} r\left(P_{5}\right)=N-r b-s+1 \text {. }
\end{aligned}
$$


Lembrando que os projetores ortogonais $\mathrm{P}_{0}, \mathrm{P}_{1}$. $P_{2}, P_{3} e P_{5}$ são matrizes simetricas e idempotentes e que $\Omega \in Y$, isto $e \quad \Omega=c_{0} P_{0}+c_{1} P_{1}+c_{2} P_{2}+c_{3} P_{3}+c_{5} P_{5}$. com $c_{i}>0$, obtem-se o sistema de equaçbes lineares:

$\left(c_{0}-c_{3}\right) P_{0}+\left(c_{1}-c_{3}\right) P_{1}+\left(c_{2}-c_{5}\right) P_{2}+\left(c_{2}-c_{5}\right) P_{4}+c_{5} I_{N}=\Omega \quad$ ou seja

$$
\begin{aligned}
& \left(c_{0}-c_{3}\right) \frac{1}{N}+\left(c_{1}-c_{3}\right) \frac{r-1}{N}+\left(c_{2}-c_{5}\right) \frac{s-1}{N}+\left(c_{3}-c_{5}\right) \frac{1}{5 K}+c_{5}= \\
& =\sigma^{2}+\alpha_{R}^{2} \\
& \left(c_{0}-c_{3}\right) \frac{1}{N}+\left(c_{1}-c_{3}\right) \frac{r-1}{N}+\left(c_{2}-c_{5}\right) \frac{5-1}{N}+\left(c_{3}-c_{5}\right) \frac{1}{5 K}= \\
& =p \sigma^{2}+\sigma^{2} \\
& \left(c_{0}-c_{3}\right) \frac{1}{N}+\left(c_{1}-c_{3}\right) \frac{r-1}{N}+\left(c_{2}-c_{5}\right)-\frac{S-1}{N}=0
\end{aligned}
$$$$
\left(c_{0}-c_{2}\right) \frac{1}{N}+\left(c_{1}-c_{3}\right) \frac{r-1}{N}+\left(c_{2}-c_{5}\right) \frac{-1}{N}+\left(c_{a}-c_{5}\right) \frac{1}{s K}=p o^{2}+o_{R}^{2}
$$$$
\left(c_{0}-c_{3}\right) \frac{1}{N}+\left(c_{1}-c_{3}\right) \frac{r-1}{N}+\left(c_{2}-c_{5}\right) \frac{-1}{N}=0
$$$$
\left(c_{0}-c_{3}\right) \frac{1}{N}+\left(c_{1}-c_{3}\right) \frac{-1}{N}+\left(c_{2}-c_{5}\right) \frac{S-1}{N}=0
$$$$
\left(c_{0}-c_{3}\right) \frac{1}{N}+\left(c_{1}-c_{3}\right) \frac{-1}{N}+\left(c_{2}-c_{5}\right) \frac{-1}{N}=0
$$ 
Sob as restriçరes paramétricas dadas por (3.11). tem-se o - seguinte quadro de Análise de Variáncia:

Quadro 3.15. Análise de variáncia para o modelo $(3.7)$ com repetiçర̃es, caso a..

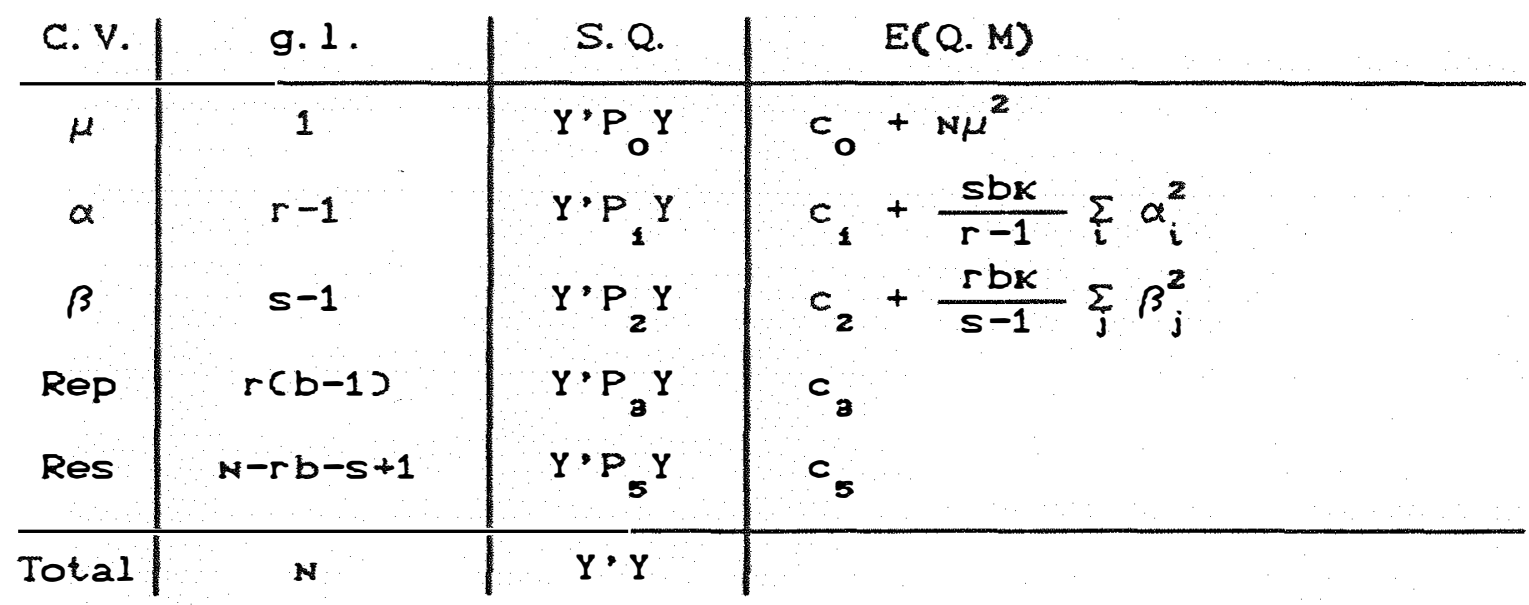

Verifica-se agora que todos os testes para as hipóteses principais podem ser feitos pelos metodos usuais, sem o comprometimento das probabilidades dos erros tipos I e II, a menos do fator repetiçశes.

Desta forma o teste de $H_{0}: \alpha_{i}=0$ ve $H_{1}: \alpha_{i} \neq 0$ para algum $i=1,2, \ldots, r$ feito em relação a "repetição". enquanto que para $H_{0}: \beta_{j}=0$ vs $H_{i}: \beta_{j} \neq 0$ para algum $j=1,2, \ldots, s$ é feito contra o "residuo", como antes.

Nota-se que para $b=1$, o fator repetição deixa de existir, pois tem-se "O" graus de liberdade para esse fator, o que se faz voltar ao mesmo problema.

- caso 3.3.1.b.2. poderia ser tratado, considerando equicorrelação dentro do niveis do fator $\beta$. ao invés de $\alpha$ " assim o fator "repetiça" passaria a ser 
hierárquizado dentro de $\beta$. compondo o modelo com a notação $R_{L(j)}$. O desenvolvimento análogo ao anterior. observando as devidas trocas de indices.

Para o caso 3.3.1.b.1. de equicorrelação dentro das caselas, em que o modelo considerado não contém interação, não foi possivel encontrar um procedimento alternativo nessa linha (incluindo repetiçöes então esse caso será tratado para modelos com interação.

b. Procedimento alternativo para o caso 3.3.2.b.1. cujo modelo inclue interação.

Consideram-se b repetiçöes de mesmo tamanho. independentes entre si e também com relaçăo aos erros $\theta_{i j l k}$ do modelo, e a inclusăo do fator repetição dentro da casela $(i, j)$, de modo que o modelo passa a ser dado por $y_{i j l k}=\mu+\alpha_{i}+\beta_{j}+\alpha \beta_{i j}+R_{l i i j\}}+e_{i j l k} \quad$ onde $i=1,2, \ldots, r, j=1,2, \ldots, s, 1=1,2, \ldots, b \quad$ e $k=1,2, \ldots, k, e R_{\text {lij) }}$ corresponde ao efeito de repetição dentro do i-ésimo nivel do fator $\alpha$ e j-ésimo nivel do fator $\beta$.

Considerando que $R_{l(i j)} \bullet \theta_{i j l k}$ são variáveis aleatórias independentes com distribuiçరes normais de medias nulas $e$ matrizes de variancias e covariancias dadas por $\Omega_{1}=\sigma_{R}^{2} I_{N}$ e $\Omega_{2}$. respectivamente, com $\Omega_{2}=I_{r s b} \otimes$ onde 


$$
\begin{aligned}
& V=\sigma^{2}\left[\begin{array}{lllll}
1 & \rho & \cdots & \rho \\
\rho & 1 & \cdots & \rho & \rho \\
0 & 0 & \cdots
\end{array}\right] r S b K \\
& \text { K }
\end{aligned}
$$

Tem-se entåo que: $\quad \operatorname{cov}\left(y_{i j l k}, y_{i / j / k}\right)=$

$=E\left[\left(y_{i j l k}-E\left(y_{i j l k}\right)\right)\left(y_{i / j / l \cdot k}-E\left(y_{i \cdot j / l \cdot k}\right)\right)\right]=$

$=\operatorname{cov}\left[\left(R_{l i j)}+\theta_{i j l k}\right)\left(R_{l \cdot(i, j,}+\theta_{i / j l k}\right)\right]=$

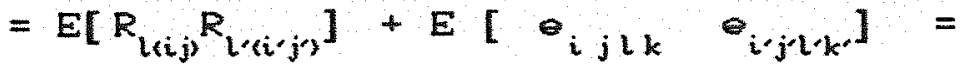

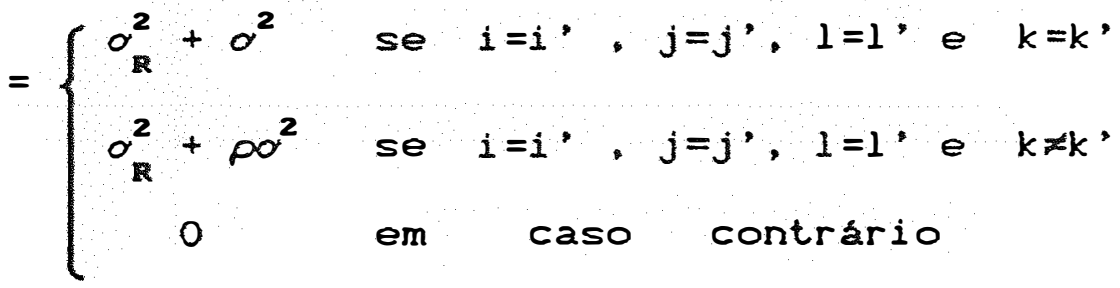

E assim a matriz de variancias e covariancias $\Omega$ do modelo é dada por:

$$
\begin{aligned}
& \Omega=V\left(\Omega_{1}, \Omega_{2}\right)=I_{r s b} \otimes V_{k}
\end{aligned}
$$

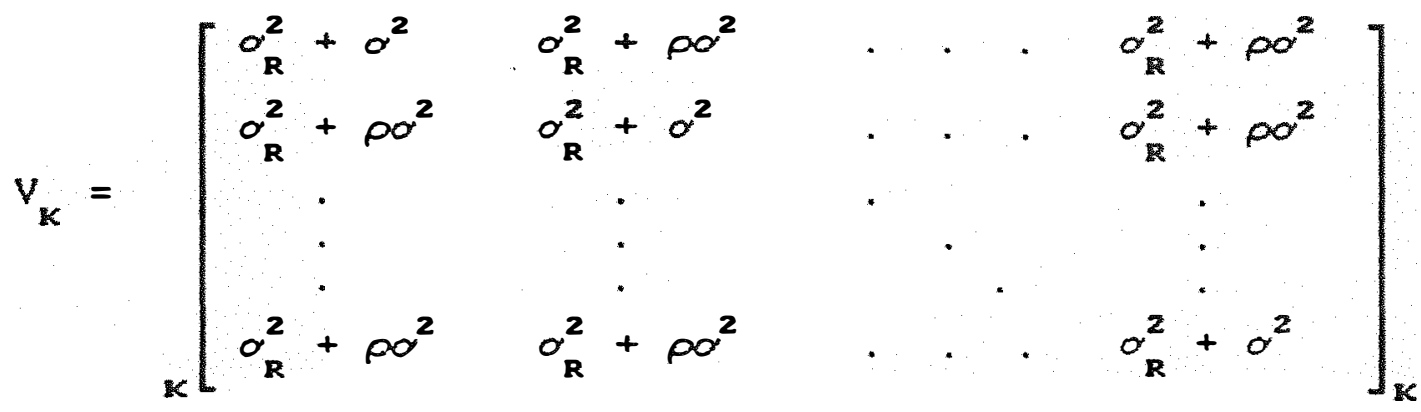

As somas de quadrados usuais para esse modelo são dadas por: 


$$
\begin{aligned}
& S Q(\mu)=Y \cdot P_{0} Y=\frac{1}{N} y^{2} \ldots \\
& S Q(\alpha)=Y \cdot P_{1} Y=\frac{1}{S b K} \sum_{i} y_{i}^{2} \ldots-\frac{1}{N} y^{2} \ldots \\
& S Q(\beta)=Y \cdot P_{2} Y=\frac{1}{r b K} \sum_{j} y^{2} \cdot j-\frac{1}{N}-y^{2} \ldots \\
& S Q(\alpha \beta)=Y \cdot P_{3} Y=\frac{1}{b k} \sum_{i j} y_{i j}^{2}-\frac{1}{S b k} \sum_{i} y_{i}^{2} \ldots-\frac{1}{r b k} \sum_{j} y^{2} j \ldots+\frac{1}{N} y^{2} \ldots \\
& S Q(R)=Y \cdot P_{i}=\frac{1}{K} \sum_{i j l} y_{i j l}^{2}-\frac{1}{b k} \sum_{i j} y_{i j}^{2} \ldots \\
& S Q(R e s i d u O)=Y \cdot P_{5} Y
\end{aligned}
$$

onde:

$$
\begin{aligned}
& P_{0}=K_{N} \\
& P_{1}=I_{r} \otimes K_{s b K}-K_{N} \\
& P_{2}=K_{r} \otimes I_{s} \otimes K_{b K}-K_{N} \\
& P_{3}=I_{r s} \otimes K_{b K}-I_{r} \otimes K_{s b K}-K_{r} \otimes I_{s} \otimes K_{b K}+K_{N}= \\
& =I_{r s} \otimes K_{b K}-P_{1}-P_{2}-P_{0}=P_{*}-P_{1}-P_{2}-P_{0} \\
& P_{4}=I_{r s b} \otimes K_{K}-I_{r s} \otimes K_{b K}=P_{* *}-P_{*} \\
& P_{5}=I_{N}-\left(P_{0}+P_{1}+P_{2}+P_{3}+P_{*}\right)=I_{N}-P_{* *} \\
& c o m=r\left(P_{0}\right)=1, r\left(P_{1}\right)=r-1, r\left(P_{2}\right)=s-1 \\
& r\left(P_{3}\right)=r s-(r-1)-(s-1)-1=(r-1)(s-1) \\
& r\left(P_{4}\right)=r s b-r s=r s(b-1) \\
& r\left(P_{5}\right)=N-r s b=r s b(k-1)
\end{aligned}
$$

$$
\text { Para que } \Omega \in \mho \text { tem-se que encontrar } c_{i}>0
$$
tal que: $\Omega=\sum_{i=0}^{5} c_{i} P_{i}$, atraves da resolução do seguinte sistema de equaçöes: 


$$
\begin{aligned}
& c_{0} P_{0}+c_{1} P_{1}+c_{2} P_{2}+c_{3} P_{3}+c_{4} P_{4} c_{5} P_{5}= \\
& =c_{0} P_{0}+c_{1} P_{1}+c_{2} P_{2}+c_{3}\left(P_{*}-P_{1}-P_{2}-P_{0}\right)+c_{4}\left(P_{* *}-P_{*}\right)+ \\
& c_{5}\left(I_{N}-P_{0}-P_{1}-P_{2}-P_{3}-P_{4}\right)=c_{0} P_{0}+c_{1} P_{1}+c_{2} P_{2}+ \\
& c_{3}\left(P_{*}-P_{0}-P_{1}-P_{2}\right)+c_{4}\left(P_{* *}-P_{*}\right)+c_{5}\left(I_{N}-P_{* *}\right)= \\
& =\left(c_{0}-c_{3}\right) P_{0}+\left(c_{1}-c_{3}\right) P_{1}+\left(c_{2}-c_{3}\right) P_{2}+\left(c_{3}-c_{4}\right) P_{*}+ \\
& +\left(c_{4}-c_{5}\right) P_{* *}+c_{5} I_{N}=\Omega \quad \text { ou seja, tem-se } 0
\end{aligned}
$$

sistema:

$$
\begin{aligned}
\left(c_{0}-c_{3}\right) \frac{1}{N}+\left(c_{1}-c_{3}\right) \frac{r-1}{N}+\left(c_{2}-c_{3}\right) & \frac{s-1}{N}+\left(c_{3}-c_{4}\right) \frac{1}{b K}+ \\
& +\left(c_{4}-c_{5}\right) \frac{1}{k}+c_{5}=\alpha^{2}+\alpha^{2}
\end{aligned}
$$

$$
\begin{aligned}
\left(c_{0}-c_{3}\right) \frac{1}{N}+\left(c_{1}-c_{3}\right) \frac{r-1}{N}+\left(c_{2}-c_{3}\right) \frac{s-1}{N} & +\left(c_{3}-c_{2}\right) \frac{1}{b K} \\
& +\left(c_{1}-c_{5}\right) \frac{1}{K}=\rho \alpha^{2}+\alpha_{R}^{2}
\end{aligned}
$$$$
\left(c_{0}-c_{3}\right) \frac{1}{N}+\left(c_{1}-c_{3}\right) \frac{r-1}{N}+\left(c_{2}-c_{3}\right) \frac{S-1}{N}+\left(c_{3}-c_{4}\right) \frac{1}{b k}=0
$$$$
\left(c_{0}-c_{3}\right) \frac{1}{N}+\left(c_{1}-c_{3}\right) \frac{r-1}{N}+\left(c_{2}-c_{3}\right) \frac{-1}{N}=0
$$$$
\left(c_{0}-c_{3}\right) \frac{1}{N}+\left(c_{1}-c_{3}\right) \frac{-1}{N}+\left(c_{2}-c_{3}\right) \frac{S-1}{N}=0
$$

$$
\left(c_{0}-c_{3}\right) \frac{1}{N}+\left(c_{1}-c_{3}\right) \frac{-1}{N}+\left(c_{2}-c_{3}\right) \frac{-1}{N}=0
$$

cuja solução é:

$$
\begin{aligned}
c_{0}=c_{1}=c_{2}=c_{3}=c_{4} & =\sigma^{2}[1+(k-1) \rho]+k \sigma_{R}^{2} \\
c_{5} & =\sigma^{2}[1-\rho]
\end{aligned}
$$


Considerando que os projetores ortogonais $\mathrm{P}_{\mathrm{i}}$ para $i=0,1,2,3,4,5$ são matrizes simetricas, idempotentes, - que $\Omega=\sum_{i=0}^{5} c_{i} P_{i}$, e sob as restriçశes paramétricas dadas em (3.18) e ainda por (3.4) (3.0). tem-se o seguinte quadro de análise de variancia:

Quadro 3.16. Análise de variancia para o modelo (3.7) com interação e repetiçós, caso b. .

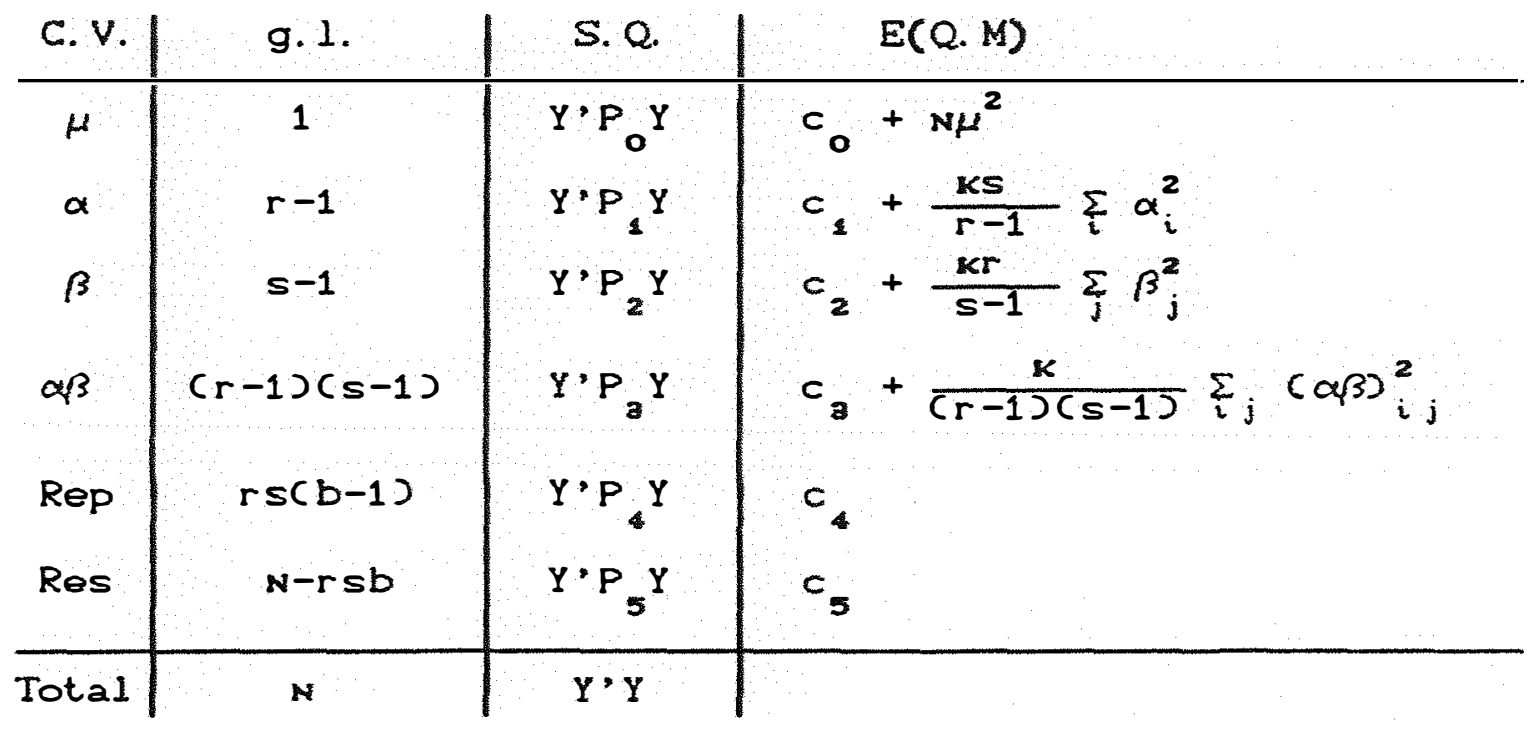

Agora os testes para as hipóteses principais podem ser feitos, pelos métodos usuais, em relação a "Repetiçă", sem comprometer as probabilidades de erros tipos I e II.

c. Procedimento Alternativo para o caso 3. 3.2. b.2.

Esse caso envolve uma equicorrelaça nas observações dentro dos niveis de um dos fatores 
independéncia com relaçăo ao outro fator. e foi tratado em 3.3.3.a. para modelos sem interaçăo. Agora para modelo com interaçăo, o procedimento e o mesmo. considerando b repetiçס̄es independentes entre si e também com relaçăo aos erros do modelo, e de mesmo tamanho.

Inclui-se no modelo o fator "repetição" dentro do fator cujas observaçôes são correlacionadas c digamos $\infty$. obtendo-se o modelo:

$$
y_{i j l k}=\mu+\alpha_{i}+\beta_{j}+\alpha \beta_{i j}+R_{l(i)}+e_{i j l k} \quad \text { onde }
$$

$i=1,2, \ldots, r, j=1,2, \ldots, s, 1=1,2, \ldots, b \quad e$ $k=1,2, \ldots, k$ e $R_{L(i)}$ corresponde ao efeito da 1 -ésima repetição dentro do i-ésimo nivel do fator $\alpha$.

Entåo $R_{l(i)} \otimes \theta_{i j l k}$ são variáveis

aleatorias independentes com distribuiçros normais de médias nulas e matrizes de variancias e covariancias $\Omega_{1}=\sigma_{R}^{2} I_{N} \quad \Omega_{2}$, respectivamente, onde $N=$ rsbK e $\Omega_{2}=I_{r} \otimes V_{s b K}=\sigma^{2} I_{r} \otimes\left[\rho\left(s K_{s} \otimes I_{b} \otimes k K_{K}\right)+(1-\rho) I_{s b K}\right]=$ $=\sigma^{2}\left[\rho I_{r} \otimes s K_{S} \otimes I_{b} \otimes K_{K}+(1-\rho) I_{N}\right]$

tem-se que: $\quad \operatorname{cov}\left(y_{i j l k}, y_{i \cdot j / l \cdot k}\right)=$

$$
\begin{aligned}
& =E\left[\left(y_{i j l k}-E\left(y_{i j l k}\right)\right)\left(y_{i / l / k}-E\left(y_{i / j l k}\right)\right)\right]= \\
& =E\left[\left(R_{l(i)}+e_{i j l k}\right)\left(R_{l^{\prime}\left(i^{\prime}\right)}+e_{i / j l / k^{\prime}}\right)\right]= \\
& =E\left[R_{L(i)} R_{L(i),}\right]+E\left[e_{i j l k} e_{i \cdot j l k^{\prime}}\right]=
\end{aligned}
$$




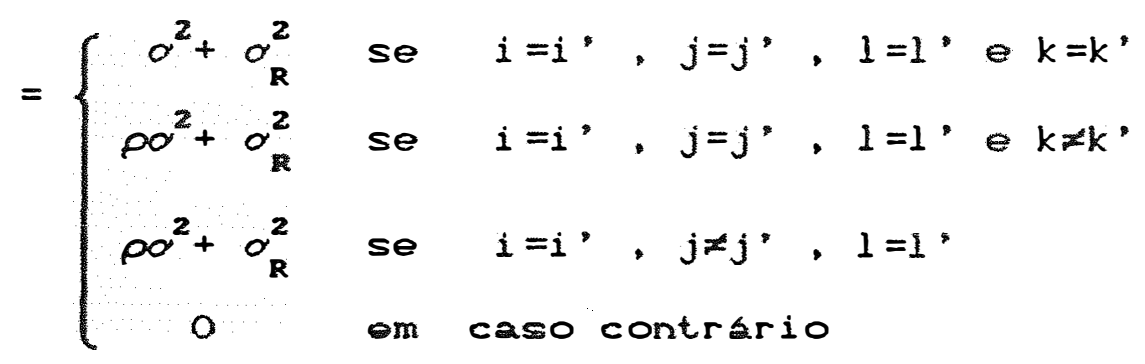

entao $\Omega=v\left(\Omega_{1} \cdot \Omega_{2}\right)=$

$$
\begin{aligned}
& =I_{r} \otimes V_{S b K}+\sigma_{R}^{2}\left[I_{S} \otimes s K_{S} \otimes I_{b} \otimes K K_{K}\right]= \\
& =\left(\rho \sigma^{2}+\sigma_{R}^{2}\right)\left[I_{r} \otimes s K_{S} \otimes I_{b} \otimes k K_{K}\right]+\sigma^{2}(1-\rho) I_{N}
\end{aligned}
$$

As somas de quadrados para os fatores desse modelo são as mesmas dadas por (3.20) em 3.3.3.b., com. exceção das SO(Repetiçăo) e SQ(Residuos) as quais são dadas por:

$S Q(R e P)=Y^{\prime} P_{4} Y=\frac{1}{S K} \sum_{i l} y_{i, l}^{2}-\frac{1}{S b K} \sum_{i} y_{i}^{2} \ldots$

onde $P_{4}=I_{r} \otimes K_{s} \otimes I_{b} \otimes K_{K}-I_{r} \otimes K_{s b K}=P_{* *}-I_{r} \otimes K_{s b K}$

$\operatorname{com} r\left(P_{4}\right)=r b-r=r(b-1)$

SOResiduo $=Y^{\prime} P_{5} Y$

onde $P_{5}=I_{N}-\left(P_{0}+P_{1}+P_{2}+P_{3}+P_{4}\right)=I_{N}-\left(P_{4}+P_{*}\right)=$ $=I_{N}-P_{* *}-P_{*}+P_{1}+P_{0}$, onde $P_{*}$ dado em (3.20) com $r\left(P_{5}\right)=N-r b-r s+r=N-r(b+s-1)$.

Para que $\Omega \in \mho$ temos que encontrar $c_{i}>0$ tal que $\quad \Omega=\sum_{i=0}^{5} c_{i} P_{i}$, através da resolução do seguinte sistema de equaçס̃es :

$c_{0} P_{0}+c_{1} P_{1}+c_{2} P_{2}+c_{3} P_{3}+c_{4} P_{4}+c_{5} P_{5}=\Omega$ 


$$
\begin{aligned}
& c_{5}\left(I_{N}-P_{4}-P_{4}+P_{1}+P_{0}\right)=\left(c_{0}-c_{3}-c_{4}+c_{5}\right) P_{0}+ \\
& +\left(c_{1}-c_{3}-c_{4}+c_{5}\right) P_{1}+\left(c_{2}-c_{3}\right) P_{2}+\left(c_{3}-c_{5}\right) P_{4}+ \\
& +\left(c_{4}-c_{5}\right) P_{*}+c_{5} I_{N}=\Omega
\end{aligned}
$$

isto e:

$$
\begin{aligned}
& \left(c_{0}-c_{3}-c_{4}+c_{5}\right) \frac{1}{N}+\left(c_{1}-c_{3}-c_{4}+c_{5}\right) \frac{r-1}{N}+\left(c_{2}-c_{3}\right) \frac{S-1}{N}+ \\
& +\left(c_{2}-c_{5}\right) \frac{1}{b K}+\left(c_{4}-c_{5}\right) \frac{1}{5 K}+c_{5}=\sigma_{R}^{2}+\sigma^{2} \\
& \left(c_{0}-c_{3}-c_{4}+c_{5}\right) \frac{1}{N}+\left(c_{1}-c_{3}-c_{4}+c_{5}\right) \frac{r-1}{N}+\left(c_{2}-c_{3}\right) \frac{S-1}{N}+ \\
& +\left(c_{3}-c_{5}\right)^{1}+\left(c_{4}-c_{5}\right) \frac{1}{5 K}=\sigma_{R}^{2}+p \sigma^{2} \\
& \left(c_{0}-c_{3}-c_{4}+c_{5}\right) \frac{1}{N}+\left(c_{1}-c_{3}-c_{4}+c_{5}\right) \frac{r-1}{N}+\left(c_{2}-c_{3}\right) \frac{5-1}{N}+ \\
& +\left(c_{3}-c_{5}\right) \frac{1}{b K}=0 \\
& \left(c_{0}-c_{3}-c_{4}+c_{5}\right) \frac{1}{N}+\left(c_{1}-c_{3}-c_{4}+c_{5}\right) \frac{r-1}{N}+\left(c_{2}-c_{2}\right) \frac{-1}{N}+ \\
& +\left(c_{4}-c_{5}\right) \frac{1}{5 K}-\quad=\sigma_{R}^{2}+p \alpha^{2} \\
& \left(c_{0}-c_{3}-c_{4}+c_{5}\right) \frac{1}{N}+\left(c_{1}-c_{3}-c_{4}+c_{5}\right) \frac{r-1}{N}+\left(c_{2}-c_{3}\right) \frac{-1}{N}=0 \\
& \left(c_{0}-c_{3}-c_{4}+c_{5}\right) \frac{1}{N}+\left(c_{1}-c_{3}-c_{4}+c_{5}\right) \frac{-1}{N}+\left(c_{2}-c_{3}\right) \frac{5-1}{N}=0 \\
& \left(c_{0}-c_{a}-c_{1}+c_{5}\right) \frac{1}{N}+\left(c_{1}-c_{a}-c_{3}+c_{5}\right) \frac{-1}{N}+\left(c_{2}-c_{3}\right) \frac{-1}{N}=0
\end{aligned}
$$

Existe uma combinação linear entre as equaçбes: segunda, terceira, quarta e quinta, então podemos eliminar. sem perda de generalidade, a quarta equação, obtendo a 
seguinte sol ução:

$$
\begin{gathered}
c_{0}=c_{1}=c_{1}=\sigma^{2}[1+(s k-1) \rho]+s k \sigma_{k}^{2} \\
c_{2}=c_{3}=c_{5}=\sigma^{2}[1-\rho]
\end{gathered}
$$

Considerando que os projetores ortogonais $P_{i}$ para $i=0,1,2,3,4$ e sao matrizes simetricas. idempotentes, e que $\Omega=\sum_{i=0}^{5} c_{i} P_{i}, \theta$ sob as restriçres parametricas dadas em (3.18) e ainda por (3.4) e (3.9) tem-se o seguinte quadro de análise de variancia:

Quadro 3.17. Análise de variancia para o modelo (3.7) com interação e repetição, caso c..

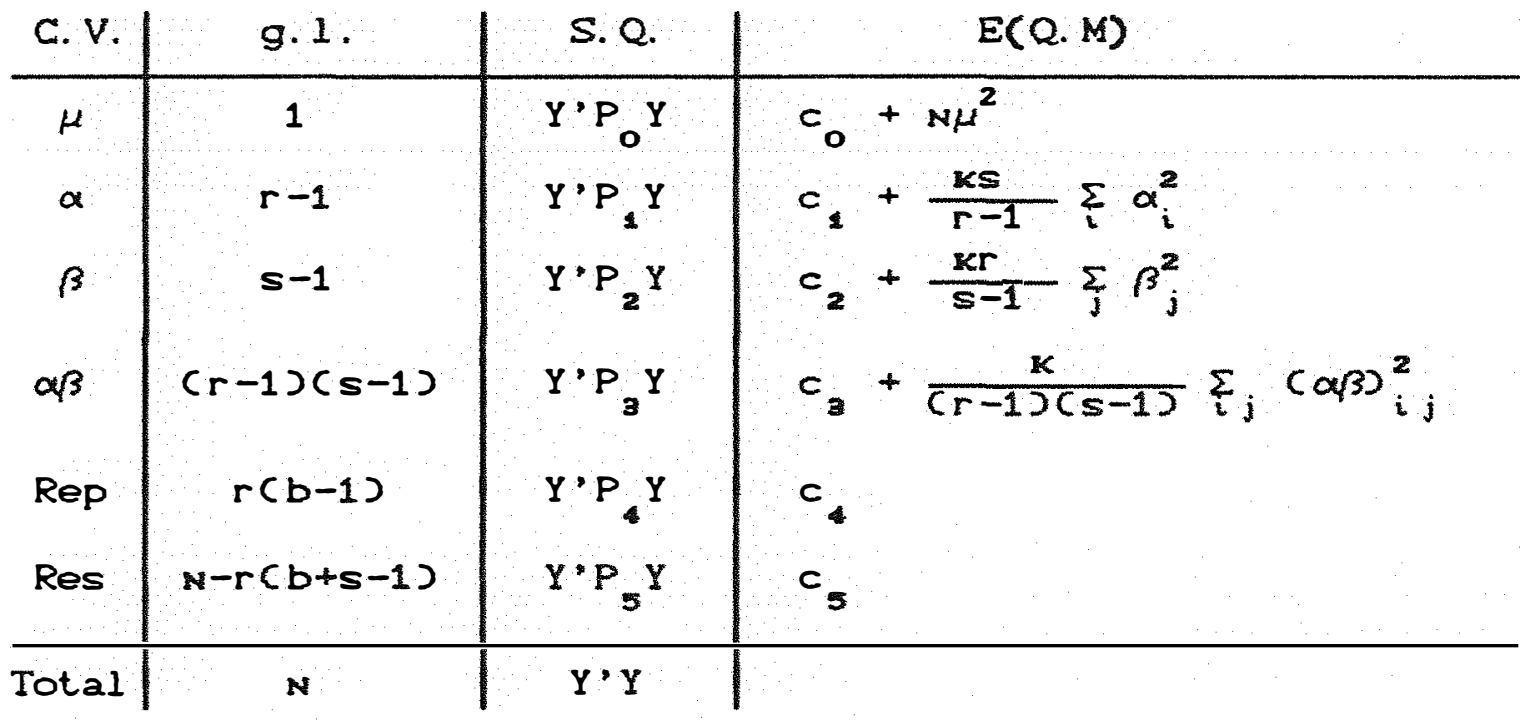

Pode-se ver que os testes para as hipoteses principais podem ser feitos, pelos métodos usuais, sem o compremetimento das probabilidades de erros tipos I e II. sendo que a hipótese $H_{0}: \alpha_{i}=0$ deve-se testá-la em rel ação a "Repetição" enquanto que para as hipóteses $H_{0}: \beta_{j}=0$ e $H_{0}: \alpha \beta_{i j}=0$ faz-se em relaçăo ao "Residuo". 


\subsection{Modelos Mistos com Dois fatores}

\subsubsection{MODELOS COM INTERACÃo}

$$
\text { Considera-se } 0 \text { modelo linear } Y=X \theta+e \text {, }
$$

caracterizado por:

$y_{i j k}=\mu+\alpha_{i}+\beta_{j}+\alpha \beta_{i j}+e_{i j k} \quad$ para $\quad$ (3.22)

$i=1,2, \ldots, r, j=1,2, \ldots, s$ e $k=1,2, \ldots, k$ e $N=r s k$

onde $\mu$ é a média geral, $\alpha_{i}$ efixo e $\beta_{j}, \alpha_{i j} e \beta_{i j k}$ săo variáveis aleatórias independentes, com distribuiçros normais de médias nulas e variancias $\alpha_{\beta}^{2}, \alpha_{\alpha \beta}^{2}$ e $\alpha^{2}$ respectivamente. Entăo $E\left(y_{i j k}\right)=\mu+\alpha_{i}$ onde $Y \in$ o vetor de observaçơses dado por (3.8)

\section{a. Erros independentes}

E usual considerar-se a estrutura de erros independentes, isto é a matriz de variancias e covariancias do vetor de erros e é dada por $\sigma^{2} I_{N}$.

Assim para o modelo dado em (3.22) obtem-se:

$$
\begin{array}{r}
\operatorname{cov}\left(y_{i j k}, y_{i \cdot j^{\prime} k^{\prime}}\right)=E\left[\left(\beta_{j}+\alpha \beta_{i j}+e_{i j k}\right)\left(\beta_{j^{\prime}}+\alpha \beta_{i j^{\prime}}+e_{i \cdot j^{\prime} k^{\prime}}\right)\right]= \\
=E\left(\beta_{j^{\prime} \beta_{j}}\right)+E\left(\alpha \beta_{i j} \alpha \beta_{i^{\prime} j^{\prime}}\right)+E\left(e_{i j k^{\prime}} e_{i j^{\prime} k^{\prime}}\right) .
\end{array}
$$


Sob as usuais restriçoes parametricas $\sum_{i} a_{i}=0$ - $\sum_{i} a \beta_{i j}=0$ para todo $j$. acarretando, por causa dessa segunda restriçăo. uma covariancia năo nula para os efeitos $a_{i j} \quad \alpha_{i-j}$ (veja SCHEFFE (1959:266-268) ou SEARLE $(1071: 401-403)$ ), isto o:

$\operatorname{var}\left(\sum_{i} \alpha \beta_{i j}\right)=\operatorname{var}(0)=0$ c pela restriça) , más

$\operatorname{var}\left(\sum_{i} \alpha \beta_{i j}\right)=\sum_{i} \operatorname{var}\left(\alpha \beta_{i j}\right)+2 \sum_{i<i} \operatorname{cov}\left(\alpha \beta_{i j}, \alpha \beta_{i j}\right)=$

$$
=r \sigma_{\alpha \beta}^{2}+2 \frac{r(r-1)}{2} c=0
$$$$
c=\operatorname{cov}\left(\alpha \beta_{i j}, \alpha \beta_{i}, j\right)=\frac{-1}{r-1} \alpha_{\alpha \beta}^{2}
$$

$$
\text { Entzo tem-se que } \operatorname{cov}\left(y_{i j k} \cdot y_{i-j k}\right)=
$$

$=E\left(\beta_{j} \beta_{j}\right)+E\left(\alpha \beta_{i j} \alpha \beta_{i \cdot j}\right)+E\left(\theta_{i j k} \theta_{i / j k}\right)=$

$=\left\{\begin{array}{ccc}\sigma^{2}+\sigma_{\beta}^{2}+\sigma_{\alpha \beta}^{2} & \text { se } & i=i, j=j, \text { e } k=k \\ \sigma_{\beta}^{2}+\sigma_{\alpha \beta}^{2} & \text { se } & i=i, j=j, \text { e } k \neq k \\ \sigma_{\beta}^{2}-\frac{1}{r-1} \sigma_{\alpha \beta}^{2} \text { se } & i \neq i, \theta j=j, \\ 0 & \text { em } c .\end{array}\right.$

e a matriz de variancias e covariancias $\Omega$ do modelo pode ser escrita na forma:

$\Omega=\sigma^{2} I_{N}+k \sigma_{\alpha \beta}^{2} I_{r s} \cdot K_{K}+r k \sigma_{\beta}^{2} K_{r} \otimes I_{S} \otimes K_{K}$

As somas de quadrados são dadas por:

$S Q(H)=Y \cdot P_{O} Y$ com $P_{O}=K_{N}$

$\operatorname{se}(\alpha)=Y \cdot P_{1} Y$ com $P_{1}=I_{r} \otimes K_{S K}-K_{N}$

$S Q(\beta)=Y \cdot P_{2} Y \quad$ com $\quad P_{2}=K_{r} \otimes I_{S} \otimes K_{K}-K_{N}$ 
$S Q(\alpha \beta)=Y \cdot P_{3} Y$ com $P_{3}=I_{r s} \otimes K_{K}-P_{0}-P_{1}-P_{2}$
SQRes $=Y \cdot P_{5} Y$ com $P_{5}=I_{N}-\left(P_{0}+P_{1}+P_{2}+P_{3}\right)=I_{N}-I_{r s} \otimes K_{K}$

$\operatorname{com} r\left(P_{0}\right)=1, r\left(P_{1}\right)=r-1, r\left(P_{2}\right)=s-2$.

$$
r\left(P_{3}\right)=(r-1)(s-1), r\left(P_{5}\right)=r s(k-1) \text { e } r\left(I_{N}\right)=N
$$

Como $\Omega=Y=f \Omega: c_{0} P_{0}+c_{1} P_{1}+c_{2} P_{2}+c_{3} P_{3}+c_{5} P_{5}$

$$
\left.c_{i}>0 \text { para } i=0,1,2,3,5\right\}
$$

tem-se $\quad c_{0} P_{0}+c_{1} P_{1}+c_{2} P_{2}+c_{9} P_{3}+c_{5} P_{5}=\Omega \quad$ ou seja $\left(c_{0}-c_{9}\right) P_{0}+\left(c_{1}-c_{9}\right) P_{1}+\left(c_{2}-c_{9}\right) P_{2}+\left(c_{9}-c_{5}\right) P_{4}+c^{2} I_{N}=\Omega$ onde $P_{*}=I_{r s} \otimes K_{K} \quad e$ obtem-se 0 seguinte sistema de equaçơes lineares:

$$
\begin{array}{ll}
\left(c_{0}-c_{3}\right) \frac{1}{N}+\left(c_{1}-c_{3}\right) \frac{r-1}{N}+\left(c_{2}-c_{3}\right) \frac{s-1}{N}+\left(c_{3}-c_{5}\right) \frac{1}{K}+c_{5}=\sigma^{2}+\alpha_{\beta}^{2}+\sigma_{\alpha \beta}^{2} \\
\left(c_{0}-c_{3}\right) \frac{1}{N}+\left(c_{1}-c_{3}\right) \frac{r-1}{N}+\left(c_{2}-c_{3}\right) \frac{s-1}{N}+\left(c_{3}-c_{5}\right) \frac{1}{K}=\alpha^{2}+\sigma^{2}+\alpha_{\alpha \beta}^{2} \\
\left(c_{0}-c_{3}\right) \frac{1}{N}+\left(c_{1}-c_{3}\right) \frac{r-1}{N}+\left(c_{2}-c_{3}\right) \frac{-1}{N} & =0 \\
\left(c_{0}-c_{3}\right) \frac{1}{N}+\left(c_{1}-c_{3}\right) \frac{-1}{N}+\left(c_{2}-c_{3}\right) \frac{s-1}{N} & =\alpha^{2}-\frac{1}{r-1} \alpha_{\alpha \beta}^{2} \\
\left(c_{0}-c_{3}\right) \frac{1}{N}+\left(c_{1}-c_{3}\right) \frac{-1}{N}+\left(c_{2}-c_{3}\right) \frac{-1}{N} & =0
\end{array}
$$

com a sol ução

$$
\begin{aligned}
c_{0}=c_{2} & =\alpha^{2}+r k \sigma_{\beta}^{2} \\
c_{1}=c_{3} & =\alpha^{2}+\frac{r k}{r-1} \alpha_{\alpha \beta}^{2} \\
c_{5} & =\alpha^{2}
\end{aligned}
$$

Portanto sob as restriçбes $\sum_{i} \alpha_{i}=\sum_{i} a \beta_{i j}=0$ apresenta-se o seguinte quadro de análise de variáncia: 
Quadro 3.18. Análise de variancia para o modelo (3.22) caso a..

\begin{tabular}{|c|c|c|c|c|}
\hline c. $\mathrm{v}$. & g. 1 . & S. Q. & E(Q.M.) & H. Assoc. \\
\hline$\mu$ & 1 & $Y \cdot P Y$ & $c_{0}+N \mu^{2}$ & \\
\hline$\alpha$ & $r-1$ & $Y \cdot P Y$ & $c_{i}+\frac{s k}{r-1} \sum_{i} \alpha_{i}^{2}$ & $H_{0}^{(4)}: a_{i}=0$ \\
\hline$\beta$ & $s-1$ & $Y \cdot P_{2} Y$ & $c_{2}$ & $H_{0}^{(2)}: \sigma_{\beta}^{2}=0$ \\
\hline$\alpha \beta$ & $(r-1)(s-1)$ & $Y \cdot P_{a} Y$ & $c_{a}$ & $H_{0}^{(B)}: \sigma_{\alpha \beta}^{2}=0$ \\
\hline Resid duo & $r s(x-1)$ & $Y \cdot P Y Y$ & $c_{5}$ & \\
\hline Total & $N$ & $Y \cdot Y$ & & \\
\hline
\end{tabular}

Assim pode-se notar que os testes são exatos (como esperávamos) para as hipóteses principais, sendo que $H_{0}^{(1)}: \alpha_{i}=0$ b testada em relaça ao $Q M(\alpha \beta)$ enquanto $H_{0}^{(2)}: \alpha_{\beta}^{2}$ e $H_{0}^{(a)}: \alpha_{\alpha \beta}^{2}=0$ são testadas em relação ao QM(Residuo).

Isto e que usualmente se faz, quando se tem a independéncia dos erros do modelo.

\section{b. Erros nåo independentes}

Os erros $e_{i j k}$ aqui é que são correlacionados, e não os efeitos $\beta_{j}$ ou $(\alpha \beta)_{i j}$ do modelo, e o são através de uma equicorrel ação. Segundo SCHEFFE (1959-sec.8.1.) se esta correlação ocorre nos efeitos dos fatores e não satisfaz a 
condiçzo de simetria Ci.e. equicorrelaça da matriz de variancias e covariancias 2 do modelo, a hipotese $H_{0}: \alpha_{i}=0$ nao pode ser testada sem erro ( nzo tem teste F exato 2 e para tal deve-se usar a estatistica $T^{2}$ de Hoteliling. Também ocorrem problemas com o poder do teste para a hipotese $H_{0}: \sigma_{\alpha \beta}^{2}=0$ pols sob a hipótese alternativa $H_{1}: \sigma_{\alpha \beta}^{2} \neq 0$ a estatistica QM( $\alpha \beta)$ /OM(Residuo) não tem distribuição $F$ central ou não central. Nă 6 o nosso caso.

Aqui tamberm estudar-se-ao dois casos de equicorrel ação dos erros, como antes.

b.1. Equicorrelaçăo das observaçơes na mesma casela

Agora o vetor de erros, para o modelo dado em (3.22), tem matriz de variáncias e covariancias dada por:

$$
\begin{aligned}
& \Omega_{e}=I \operatorname{rs}^{\otimes} \mathrm{V} \quad \text { onde } \\
& V=\sigma^{2}\left[\begin{array}{ccccc}
1 & \rho & \cdot & \cdot & \rho \\
\rho & 1 & \cdot & \cdot & \rho \\
: & : & \cdot & . & \vdots
\end{array}\right] \text { entao tem-se }
\end{aligned}
$$

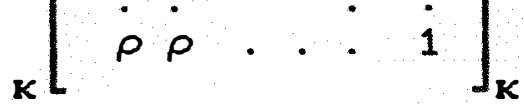

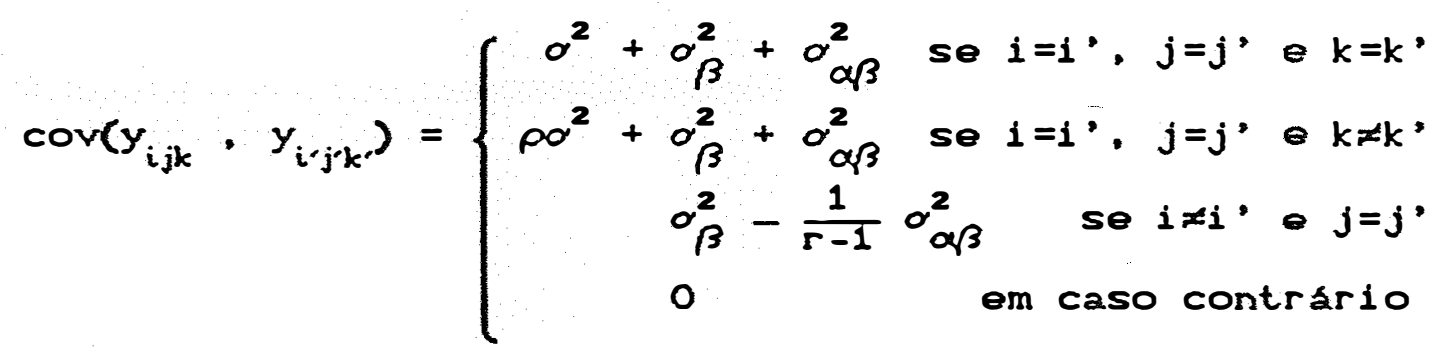


- a matriz $\Omega$ de variancias e covariancias do modelo passa a ser dada por:

$$
\begin{aligned}
\Omega= & \sigma^{2}\left[I_{r S} \otimes k \rho K_{K}+(1-\rho) I_{N}\right]+k\left(\sigma_{\alpha \beta}^{2}+\frac{1}{r-1} \sigma_{\sigma \beta}^{2} I_{r S} \otimes K_{K}\right. \\
& +r k\left(\sigma_{\beta}^{2}-\frac{1}{r-1} \sigma_{\alpha \beta}^{2}\right) K_{r} \otimes I_{S} \otimes K_{K}
\end{aligned}
$$

As somas de quadrados YP Y assim como as matrizes $P_{i}$ para $i=0,1,2,3$ e 5 sao dadas por (3.23). Como $\Omega \in \mathcal{Z}$, veja (3.24), ontao podomos encontrar os valores $c_{i} C_{1}=0,1,2,3$ e 5$)$ através de um sistema de equaços li neares como temos feito até agora, ou então utilizando a expressão dada em (3.26) para $\Omega$ isto é:

$$
\begin{aligned}
& \Omega=\sigma^{2}\left[I_{r S} \otimes \operatorname{kpK}_{K}+(1-\rho) I_{N}\right]+\frac{k r}{r-1} \alpha_{\alpha \beta}^{2} I_{r S} \otimes K_{K}+ \\
& +\left(r K \sigma_{\beta}^{2}-\frac{r K}{r-1} \sigma_{\alpha \beta}^{2}\right) K_{r} \otimes I_{S} \otimes K_{K}=\sigma^{2}(1-\rho) I_{N}+ \\
& +\left(k p \sigma^{2}+\frac{k r}{r-I} \sigma_{\alpha \beta}^{2}\right) I_{r s} \otimes K_{K}+\left(r k \sigma_{\beta}^{2}-\frac{k r}{r-1} \sigma_{\alpha \beta}^{2}\right) \\
& K_{r} \otimes I_{s} \otimes K_{k}=\sigma^{2}(1-P)\left[P_{0}+P_{1}+P_{2}+P_{j}+P_{s}\right]+ \\
& +\left(k p \sigma^{2}+\frac{k r}{r-1} \sigma_{\alpha \beta}^{2}\right)\left[P_{3}+P_{0}+P_{1}+P_{2}\right]+\left(r k \sigma_{\beta}^{2}-\frac{k r}{r-1} \alpha_{\alpha \beta}^{2}\right) \\
& {\left[P_{0}+P_{2}\right]=\left(\sigma^{2}[1+(k-1) \rho]+r k \sigma_{\beta}^{2}\right) P_{0}+} \\
& \left(\sigma^{2}[1+(k-1) \rho]+\frac{r k}{r-1} \sigma_{\alpha \beta}^{2}\right) P_{1}+ \\
& +\left(\sigma^{2}[1+(k-1) \rho]+r k \sigma_{\beta}^{2}\right) P_{2}+ \\
& +\left(\sigma^{2}[1+(k-1) \rho]+\frac{r k}{r-1} \sigma_{\alpha \beta}^{2}\right) P_{a}+ \\
& +\alpha^{2}(1-p) P_{5}=c_{0} P_{0}+c_{1} P_{1}+c_{2} P_{2}+c_{3} P_{3}+c_{5} P_{5} \text {. }
\end{aligned}
$$

logo tem-se:

$$
\begin{aligned}
& c_{0}=c_{2}=\sigma^{2}[1+(k-1) \rho]+r k \sigma_{\beta}^{2} \\
& c_{1}=c_{3}=\sigma^{2}[1+(k-1) \rho]+\frac{r k}{r-1} \sigma_{\alpha \beta}^{2} \\
& c_{5}=\alpha^{2}[1-\rho] \quad \operatorname{com} \frac{-1}{k-1}<\rho<1
\end{aligned}
$$


Assim o quadro de análise de variáncia é dado por:

Quadro 3.19. Análise de variancia para o modelo (3.22). caso b. 1..

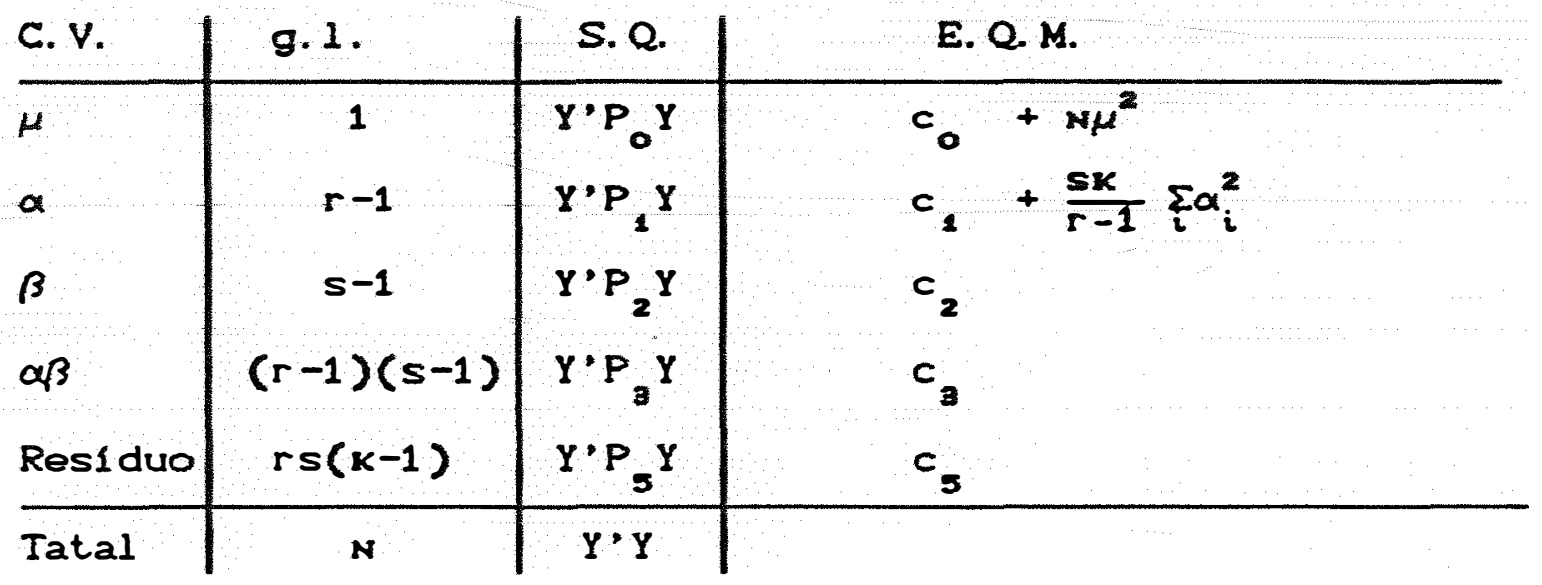

Pode-se notar que o unico teste que pode ser feito exatamente 60 da hipotese $H_{0}^{(1)}: \alpha_{i}=0$ ve $H_{1}^{(1)}: \alpha_{i} \neq 0$ para algum $i$. Os demais testes para as hipóteses principais, não podem ser feitos de maneira usual sem que haja al teraçôes das probabilidades dos erros tipos I e II.

Para as hipóteses $H_{0}^{(2)}: \alpha_{\beta}^{2}=0$ e $H_{0}^{(a)}: \alpha_{\alpha \beta}^{2}=0$ tem-se que o teste usual leva nos as seguintes probabilidades:

$P($ erro tipo $I)=P\left[F_{i 5}\left(c_{i}, c_{5}\right) \geq \frac{c_{5}}{c_{i}} F_{\alpha}\left(r\left(P_{i}\right) ; \operatorname{rs}(k-1)\right)\right] \geq \alpha$ isto e P(erro tipo I) $\left\{\begin{array}{ccc}>\alpha & \text { se } & 0<\rho<1 \\ =\alpha & \text { se } & \rho=0 \\ <\alpha & \text { se } & \frac{-1}{k-1}<\rho<0\end{array}\right.$ para $i=2,3$ onde $\alpha$ o nivel de significancia supostamente correto usado pelo pesquisador. 


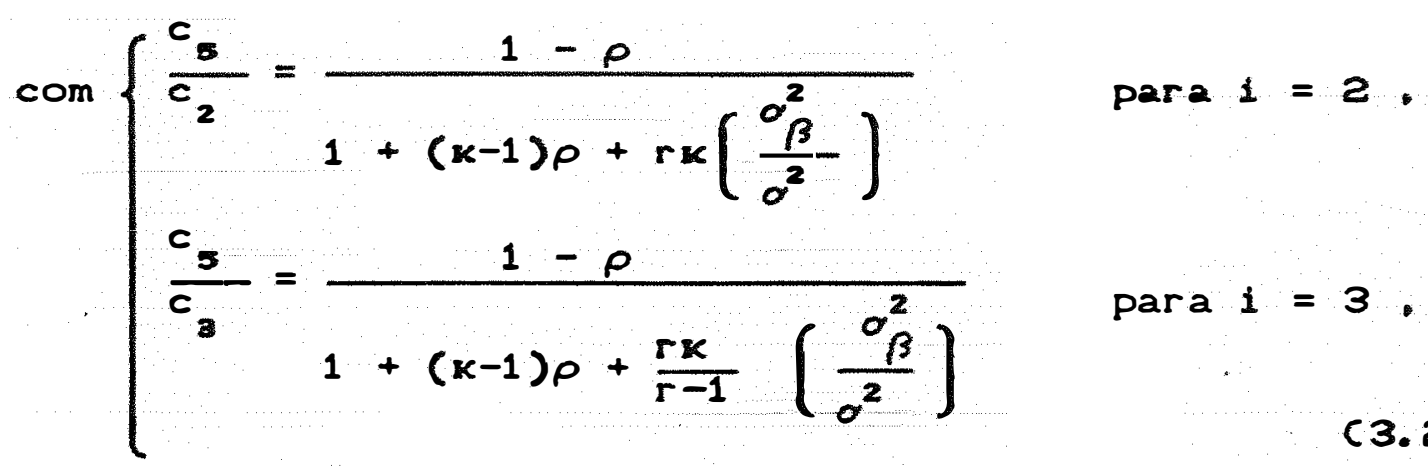

pois usualmente essas hipoteses são testadas contra o residuo.

$$
\text { Entao sob } H_{0}^{(i)}: \alpha_{\beta}^{2}=0 p / i=20 \alpha_{\alpha \beta}^{2}=0
$$

$p^{\prime} i=3$. tem-se $\frac{c_{5}}{c_{2}}=\frac{c_{5}}{c_{a}}=\frac{1-\rho}{1+c_{k-15 p}}$ e as tabelas para as P(erro tipo I) ao nIvel nominal $\alpha=0.05$, são as mesmas dadas pela IABELA A.9. do apendice para $H^{(2)}$ e $H^{(3)}$.

$$
\text { Na verdade como } c_{i}>0 \text { para } i=0,1,2,3 \text { e } 5
$$

tem-se $\rho>\max \frac{1}{k-1} 1-\left(1+r k \frac{\alpha_{\beta}^{2}}{\alpha^{2}}\right) ;-\left(1+\frac{r k}{-1}-\frac{\sigma^{2}}{\sigma^{2}}\right) 1$

mas em geral os valores $\frac{\sigma_{\beta}^{2}}{\sigma^{2}} \quad e \frac{\sigma_{\alpha \beta}^{2}}{\sigma^{2}}$ nåo são conhecidos, entå toma-se $\frac{-1}{k-1}<\rho<1$, afim de se assegurar a variação de $p$.

Quanto às probabilidades do erro tipo II tem-se que sob $H_{1}^{(i)}$ para $i=2,3$ :

$P($ erro tipo II $)=P\left[F_{i 5}\left(c_{i}, c_{5}\right)<\frac{c_{5}}{c_{i}} F_{\alpha}\left(r\left(P_{i}\right) ; r S(k-1)\right)\right]$ onde $\frac{c_{5}}{c_{i}}$ são dados por (3.27) para $i=2$ e 3 .

Essas probabilidades podem facilmente ser 
encontradas, analogamente à IABELA A.Z. do apéndice atribuindo-se valores para $\frac{\sigma_{\beta}^{2}}{\alpha^{2}} \in \operatorname{para} \frac{\sigma_{\alpha \beta}^{2}}{\sigma^{2}}$.

b.2. Equicorrelação das observaçŏes dentro dos niveis do fator " $\alpha$ "

Neste caso o vetor de erros, para o modelo dado em (3.22), tem matriz de variancias e covariancias dada por $\Omega_{e}=I_{r} \otimes V$ onde

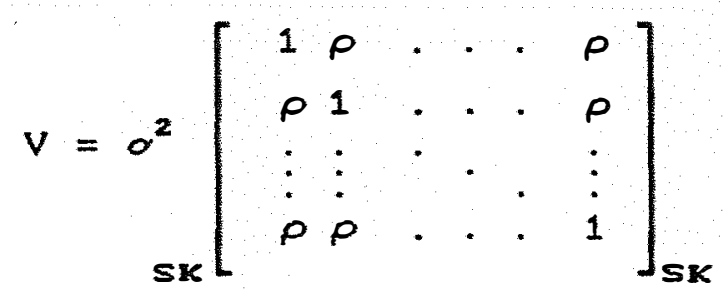

Obtendo-se

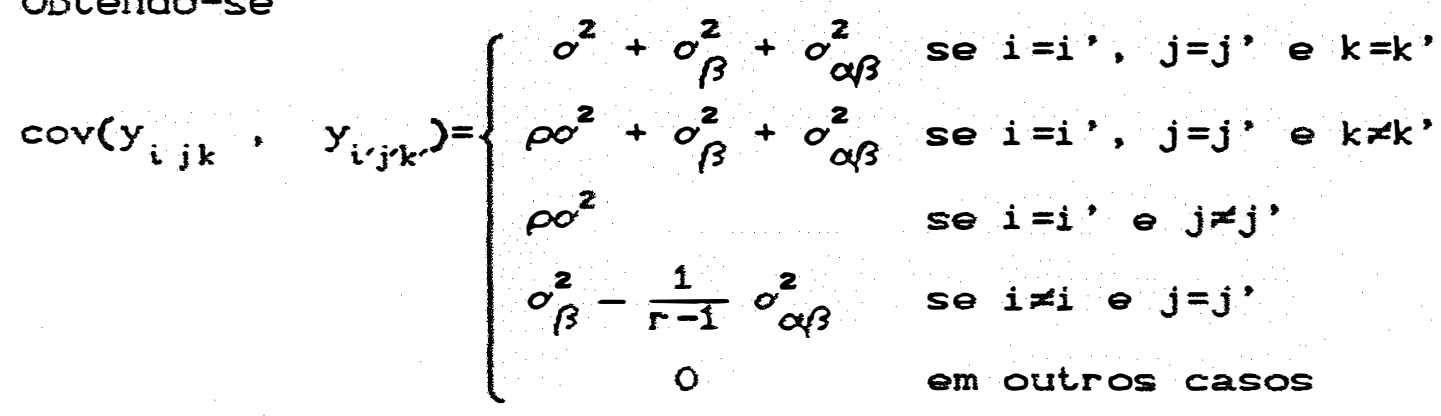

e a matriz $\Omega$ de variancias e covariancias do modelo passa a ser dada por: 


$$
\begin{aligned}
& \Omega=\alpha^{2}\left(I_{r} \operatorname{skpK}_{S K}+(1-\rho) I_{N}\right)+k\left(\alpha_{\alpha \beta}^{2}+\frac{1}{r}=\alpha_{\alpha \beta}^{2}\right) I_{r S} \otimes K_{K}+ \\
& +r K\left(\sigma_{\beta}^{2}-\frac{1}{4} \sigma_{\alpha \beta}^{2}\right) K_{r} \otimes I_{S} \otimes K_{K}=\sigma^{2}(1-\rho) I_{N}+ \\
& +\operatorname{skp}^{2} I_{r} \odot K_{S K}+\frac{r K}{r-I} \alpha_{\alpha \beta}^{2} I_{r S} \otimes K_{K}+ \\
& +\left(r K \sigma_{\beta}^{2}-\frac{r K}{r-1} \alpha_{\alpha \beta}^{2}\right) K_{K} \otimes I_{S} K_{K}=\alpha^{2}(1-\rho)\left[P_{0}+P_{1}+P_{2}+P_{3}+P_{5}\right]+ \\
& +\operatorname{skp\sigma ^{2}}\left[P_{0}+P_{1}\right]+\frac{r k}{r-1} \sigma_{\alpha \beta}^{2}\left[P_{0}+P_{1}+P_{2}+P_{3}\right]+ \\
& +\left(r k \sigma_{\beta}^{2}-\frac{r k}{r-1} \alpha_{\alpha \beta}^{2}\right)\left[P_{0}+P_{2}\right]= \\
& =\left(\alpha^{2}[1+(s k-1) \rho]+r k \sigma_{\beta}^{2}\right) P_{0}+\left(\alpha^{2}[1+(s k-1) \rho]+\frac{r k}{r-1} \sigma_{\alpha \beta}^{2}\right) P_{1}+ \\
& +\left(\alpha^{2}[1-\rho]+r k \alpha_{\beta}^{2}\right) P_{2}+\left(\alpha^{2}[1-\rho]+\frac{r k}{r-1} \alpha_{\alpha \beta}^{2}\right) P_{2}+\alpha^{2}[1-\rho] P_{5}= \\
& =c_{0} P_{0}+c_{1} P_{1}+c_{2} P_{2}+c_{3} P_{3}+c_{5} P_{5}
\end{aligned}
$$

\begin{tabular}{|c|c|c|c|c|}
\hline c. $v$. & 9.1 . & S. Q. & $E(Q . M)$. & H. Associadas \\
\hline$\mu$ & 1 & $Y^{\prime} P Y$ & $c_{0}+N \mu^{2}$ & \\
\hline$\alpha$ & $r-1$ & $Y \cdot P_{1} Y$ & $c_{1}+\frac{s K}{r-1} \sum_{i} \alpha_{i}^{2}$ & $H_{0}^{(1)}: \alpha_{i}=0$ \\
\hline$\beta$ & $s-1$ & $Y P_{2} Y$ & $c_{2}$ & $\mathrm{H}_{0}^{(2)}: \sigma_{\beta}^{2}=0$ \\
\hline$\alpha \beta$ & $(r-1)(s-1)$ & $Y \cdot P_{8} Y$ & $c_{3}$ & $H_{0}^{(a)}: \alpha_{\alpha \beta}^{2}=0$ \\
\hline Residuo & $r s(k-1)$ & $Y \cdot P_{5} Y$ & $c_{5}$ & \\
\hline Total & $N$ & $Y * Y$ & & \\
\hline
\end{tabular}

logo tem-se: $\quad c_{0}=\sigma^{2}[1+\operatorname{csk}-12 \rho]+r k \sigma_{\beta}^{2}$

$$
\begin{aligned}
& \left.c_{1}=\sigma^{2}\left[1+c_{k}-1\right) \rho\right]+\frac{r k}{r-1} \sigma_{\alpha \beta}^{2} \\
& c_{2}=\sigma^{2}[1-\rho]+r k \sigma_{\beta}^{2} \\
& c_{3}=\sigma^{2}[1-\rho]+\frac{r k}{r-1} \sigma_{\alpha \beta}^{2} \\
& c_{5}=\sigma^{2}[1-\rho]
\end{aligned}
$$

e o quadro de análise de variáncia será dado pelo:

Quadro 3.20. Análise de variáncia para o modelo (3.22), caso b. Z. . 
Pode-se notar que os testes para as hipoteses principais $H_{0}^{(2)}: \sigma_{\beta}^{2}=0 \quad H_{0}^{(3)}: \alpha_{\alpha \beta}^{2}=0$ podem ser feitos pelos métodos usuais, sem o comprometimento das probabilidades dos erros tipo I.

Para a hipotese $H_{0}: \alpha_{i}=0$ ve $H_{i}: \alpha_{i} \neq 0$ para al gum $i=1,2 \ldots . r$ isso não ocorre e a P(erro tipo I) é dada por :

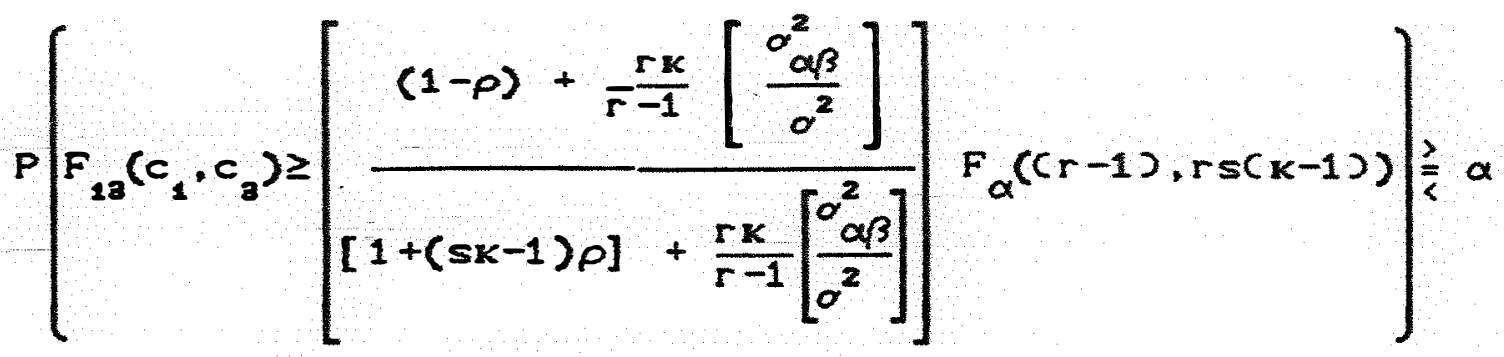

uma vez que essa hipótese e usual mente testada contra a interação, isto é

$$
\text { P(erro tipo I) }\left\{\begin{array}{llll}
>\alpha & \text { se } & \frac{-1}{s k-1}<\rho<0 \\
=\alpha & \text { se } & \rho=0 \\
<\alpha & \text { se } & \rho>0
\end{array}\right.
$$

Para obter-se a probabilidade acima é preciso que os valores de $\alpha^{2}$ e $\sigma_{\alpha \beta}^{2}$, alem de $\rho$. sejam conhecidos Assim para construir-se uma tabela dessas probabilidades pode-se atribuir valores conhecidos a esses parametros, ou melhor para a razao $\left(\frac{\alpha_{\alpha \beta}^{2}}{\sigma^{2}}\right)$.

Quanto as probabilidades dos erros tipo II. tem-se que sob $H_{1}^{(1)}: \alpha_{i} \neq 0$ para algum $i=1,2, \ldots, r$. geralmente $\lambda_{1}=\frac{1}{2 c_{1}} E(Y,) P_{1} E(Y) \neq 0 \quad, \quad e$ assim 
$P($ erro tipo II $)=P\left(F_{13}\left[c_{1}, c_{3}, \lambda_{1}, 0\right]<\frac{c_{a}}{c_{1}} F_{\alpha}(r-1, \operatorname{rs}(k-1))\right)$

onde $\frac{c_{a}}{c_{1}}=\left[(1-\rho)+\frac{r k}{r}-I\left[\frac{\alpha^{2}}{\alpha^{2}}\right]\right],\left[(1+(s k-1) \rho)+\frac{r k}{r-1}\left[\frac{\alpha^{2}}{\alpha^{2}}\right]\right]$

e devem ser calculadas, dando-se valores para $\frac{\sigma_{\alpha \beta}^{2}}{\sigma^{2}}$, e $\lambda_{1}$ Observarse que se $\alpha_{\alpha \beta}^{2}=0$ entå as probabilidades dos erros tipo I são as mesmas dadas na TABELA A.11. do apéndice, e dos erros tipo I I são dadas pela TABELA A.12. do apéndice, para os devidos valores de $r, s$, K. $\rho \in \lambda$.

\subsubsection{Procedimentos Alternativos}

Seja o caso 3.4.1.b.1. de equicorrel ação das observaçres na mesma casela com b repetiçơes independentes e de mesmo tamanho do delineamento. Então o modelo (3.22) passa a ser dado por:

$y_{i j l K}=\mu+\alpha_{i}+\beta_{j}+\alpha \beta_{i j}+R_{l(i j)}+e_{i j l k} \quad$ onde $R_{\text {l(ij) }} \cap N$ NOO $\sigma_{R}^{2}$ e são independentes das demais distribuiç̧es envolvidas no modelo, e corresponde ao efeito de repetição dentro da casela $(i, j)$, para $i=1,2, \ldots, r, j=1,2, \ldots, s, \quad 1=1,2, \ldots, b \quad e$ $k=1,2, \ldots, k, N=$ rsbk. 
Tem-se que a covariancia entre duas observaçóes quaisquer ó dada por:

$$
\begin{aligned}
& \operatorname{cov}\left(y_{i j l k} \cdot y_{i \cdot j \cdot l \cdot k}\right)= \\
& =E\left[\left(\beta_{j}+\alpha \beta_{i j}+R_{l(i, j\rangle}+e_{i j l k}\right)\left(\beta_{j \cdot}+\alpha \beta_{i \cdot j},+R_{l \cdot\left\{i \cdot j \cdot \gamma^{\prime}\right.}+e_{i \cdot j \cdot l \cdot k}\right)\right] \\
& =E\left(\beta_{j} \beta_{j},\right)+E\left(\alpha \beta_{i j} \alpha \beta_{i \cdot j}\right)+E\left(R_{l i, j}, R_{l \cdot(i \cdot j \cdot)}\right)+ \\
& +E\left(e_{i j l k} e_{i \cdot j \cdot l \cdot k},\right)=
\end{aligned}
$$



As somas de quadrados sao dadas por:

$$
\begin{aligned}
& S Q(\mu)=Y \cdot P_{0} Y=\frac{1}{N} y^{2} \ldots \quad \operatorname{com} P_{O}=K_{N} \\
& S Q(a)=Y \cdot P_{1} Y=\frac{1}{S b K} \sum_{i} y_{i}^{2} \ldots-\frac{1}{N} y^{2} \ldots \operatorname{com} P_{1}=I_{r} K_{s b K} K_{N} \\
& S Q(\beta)=Y \cdot P_{Z} Y=\frac{1}{r b K} \sum_{j} y^{2} j \ldots-\frac{1}{N} y^{2} \ldots \operatorname{com} P_{2}=K_{r} \otimes I_{b K}-K K_{N} \\
& \operatorname{so}(\alpha \beta)=Y \cdot P_{a} Y=\frac{1}{b K} \sum_{i j} y_{i j}^{2} .-\frac{1}{s b K} \sum_{i} y_{i}^{2} \ldots-\frac{1}{r b K} \sum_{j} y_{j}^{2} \ldots+ \\
& +\frac{1}{N} y^{2} \ldots \quad c o m P_{a}=I_{r s} \odot K_{b K}-P_{1}-P_{2}-P_{0}=P_{*}-P_{0}-P_{1}-P_{2} \\
& \text { SORepeti ̧ão }=Y \cdot P Y=\frac{1}{K} \sum_{i j l} y_{i j l}^{2}-\frac{1}{b K} \sum_{i j} y_{i j}^{2} . \quad \text { com } \\
& P_{4}=I_{r s b} \otimes K_{K}-I_{r s} \otimes K_{b K}
\end{aligned}
$$


SOResiduo $=Y \cdot P_{5} Y$ com $P_{5}=I_{N}-\left(P_{0}+P_{1}+P_{2}+P_{3}+P_{4}\right)$.

Sendo $\quad \Omega \in \mho \quad \Omega=\sum_{i=0}^{5} c_{i} P_{i} \quad$ ou seja

$\Omega=c_{0} P_{0}+c_{1} P_{1}+c_{2} P_{2}+c_{3} P_{3}+c_{4} P_{4}+c_{5} P_{5}$

$\Omega=\left(c_{0}-c_{3}\right) P_{0}+\left(c_{1}-c_{3}\right) P_{1}+\left(c_{2}-c_{3}\right) P_{2}+\left(c_{3}-c_{5}\right) P_{4}+$

$+\left(c_{4}-c_{5}\right) P_{4}+c^{5} I_{N}$

obtendo-se o sistema de equaçßes lineares:

$$
\begin{aligned}
& \left(c_{0}-c_{3}\right) \frac{1}{N}+\left(c_{1}-c_{3}\right) \frac{\Gamma-1}{N}+\left(c_{2}-c_{3}\right) \frac{s-1}{N}+\left(c_{3}-c_{5}\right) \frac{1}{b K}+ \\
& +\left(c_{1}-c_{5}\right) \frac{b-1}{b k}+c_{5}=\alpha^{2}+\alpha_{\beta}^{2}+\alpha_{\alpha \beta}^{2}+\alpha_{R}^{2} \\
& \left(c_{0}-c_{3}\right) \frac{1}{N}+\left(c_{1}-c_{3}\right) \frac{r-1}{N}+\left(c_{2}-c_{3}\right) \frac{S-1}{N}+\left(c_{3}-c_{5}\right) \frac{1}{b K}+ \\
& +\left(c_{4}-c_{5}\right) \frac{b-1}{b K}+\cdots=\rho \alpha^{2}+\alpha_{\beta}^{2}+\alpha_{\alpha \beta}^{2}+\alpha_{R}^{2} \\
& \left(c_{0}-c_{3}\right) \frac{1}{N}+\left(c_{1}-c_{3}\right) \frac{r-1}{N}+\left(c_{2}-c_{3}\right) \frac{5-1}{N}+\left(c_{3}-c_{5}\right) \frac{1}{b K}+ \\
& +\left(c_{4}-c_{5}\right) \frac{-1}{b K}=\alpha_{\beta}^{2}+\alpha_{\alpha \beta}^{2} \\
& \left(c_{0}-c_{3}\right) \frac{1}{N}+\left(c_{1}-c_{3}\right) \frac{r-1}{N}+\left(c_{2}-c_{3}\right) \frac{-1}{N}+=0 \\
& \left(c_{0}-c_{3}\right) \frac{1}{N}+\left(c_{1}-c_{3}\right) \frac{-1}{N}+\left(c_{2}-c_{3}\right) \frac{S-1}{N}+=\sigma_{\beta}^{2}-\frac{1}{r-1} \sigma_{\alpha \beta}^{2} \\
& \left(c_{0}-c_{3}\right) \frac{1}{N}+\left(c_{1}-c_{3}\right) \frac{-1}{N}+\left(c_{2}-c_{3}\right) \frac{-1}{N}+\cdots=0
\end{aligned}
$$

cuja solução é :

$$
\begin{aligned}
c_{1}=c_{3} & =\sigma^{2}[1+(k-1) \rho]+\frac{r b k}{r-1} \sigma_{\alpha \beta}^{2}+k \sigma_{R}^{2} \\
c_{2}=c_{0} & =\sigma^{2}[1+(k-1) \rho]+r b_{k} \sigma_{\beta}^{2}+k \sigma_{R}^{2} \\
c_{4} & =\sigma^{2}[1+(k-1) \rho]+k \sigma_{R}^{2} \\
c_{5} & =\sigma^{2}[1-\rho]
\end{aligned}
$$


Sob as restriçбes parametricas $\sum_{i} a_{i}=\sum_{i} a \beta_{i j}=0$, tem-se - seguinte quadro de análise variáncia:

Quadro 3.21. Análise de variáncia para o modelo (3.22) com repetiçర̃es, caso 3.4.2.

\begin{tabular}{|c|c|c|c|c|}
\hline c. v. & g. 1. & S. $Q$ & $E(Q . M)$. & H. Associ adas \\
\hline$\mu$ & 1 & $Y P_{O} Y$ & $c_{0}+N \mu^{2}$ & \\
\hline$\alpha$ & $r-1$ & $Y \cap P_{Y} Y$ & $c_{1}+\frac{5 K}{r-1} \sum_{i} \alpha_{i}^{2}$ & $H_{0}^{(1)}: \alpha_{i}=0$ \\
\hline$\beta$ & $s-1$ & $Y \cdot P_{2} Y$ & $c_{2}$ & $H_{0}^{(2)}: \sigma_{\beta}^{2}=0$ \\
\hline$a \beta$ & $(r-1)(s-1)$ & $Y \cdot P_{3} Y$ & $c_{a}$ & $H_{0}^{(a)}: \sigma_{\alpha \beta}^{2}=0$ \\
\hline Rep. & $r s(b-1)$ & $Y \cap P Y$ & $c_{4}$ & $H_{0}^{(4)}: \sigma_{R}^{2}=0$ \\
\hline Residuo & $N-r s b$ & $Y \cdot P_{5} Y$ & $c_{5}$ & \\
\hline Total & $\mathbf{N}$ & $Y: Y$ & & \\
\hline
\end{tabular}

Entao pode-se ver que os testes para as hipóteses principais $H^{(1)} \cdot H^{(2)} \odot H^{(3)}$. podem ser feitos pelos métodos usuais, sem o comprometimento do nivel de significancia pro-fixado, isto el para $H^{(1)}, H^{(2)} \otimes H^{(3)}$ utiliza-se respectivamente as estatisticas: OMC 0 /OMK $\alpha \beta$. OMC $\beta) / Q M(R e p$.$) e OMC \alpha \beta)$ OMCRep. J. Mas para $H^{(4)}$ não se tem teste exato, segundo essa metodologia.

Para o caso 3.4.1.b.2. de equicorrelą̧ăo das observaçర̃es dentro dos niveis do fator " $\alpha$ ", năo se obtem resultados satisfatórios com a utilização de repetiçరes do experimento, pois a hipótese em que se tem o interesse de eliminar o viés "H(1)". é a única que ainda permanece 
com viés no novo modelo. isto e: de nada adianta planejar o experimento com repetiçбes. Portanto houve-se por bem não discutir aqui os resultados obtidos com o novo modelo. 


\section{RESULTADOSE DISCUSSÃO}

Como principal resultado desse trabalho. destacam-se as tabelas publicadas no apendice e que mostram os vieses nas probabilidades dos erros tipos I e II. para os vários modelos aqui considerados e segundo os tipos de equicorrelaçăo. Săo 14 tabelas que se subdividem em 28. para os casos aqui tratados. Cada tabela fornece as probabilidades dos erros para 7 valores de " $\rho$ ", de modo que a matriz de variancias e covariancias seja positiva definida, segundo os vários valores de r.s.e k. No caso das probabilidades dos erros tipo II, foram considerados tres valores para o parámetro de não centralidade $\lambda$, a saber $\lambda=0, \lambda=5$ e $\lambda=20$. Para esses valores de $, \quad r, s, k$. e $\lambda$ Cou próximo destess. o pesquisador podera avaliar o nivel de significáncia e o poder de seus testes, caso se utilize dos testes $F$ da análise de variáncia univariada usual (isto $\&$, para erros independentes), ao nível nominal de significancia $\alpha=0,05$. 
Para cada modelo para cada caso de equicorrelaçăo. mostram-se quais hipóteses apresentam viós nas suas probabilidades de erro e também. quando é possivel, como a inclusăo de repetiçơes no modelo nos leva a testes exatos para as hipóteses principais.

Por todo o trabalho, destaca-se também a metodologia enfocada na construção da análise de variancia. segundo a classe $¥$ de matrizes de variancias e covariancias. Não se pode deixar de citar aqui a maneira pela qual foram obtidas as probabilidades dos erros tipo II. uma vez que não se tinha até o momento informaçßes de fontes geradoras de tais probabilidades, pois as tabelas com base nas probabilidades de TANG (1938), ou mesmo os cartóes gráficos de PEARSON e HARTLEY (1951), ou de FOX (1956) para o poder do teste $F$, não resolviam nosso problema.

Pretendeu-se que os resultados desse trabalho tivessem inlcio com o primeiro caso de equicorrelação aqui tratado, pela metodologia empregada na construção da análise de variáncia, pelos métodos alternativos propostos, pelos metodos utilizados nos cálculos das probabilidades dos erros tipos I e II e finalmente pelas tabelas que estão descritas no apéndice.

A classe $Z$ de matrizes de variáncias e covariáncias, considerada para todos os modelos, permite a construção da análise de variancia utilizando matrizes, com certa facilidade. para a maioria dos planejamentos mais 
usuais. Com a variação dos coeficientes $\left.c_{i}\left(c_{i}\right) 0\right)$, pode-se tomar matrizes bem gerais de variancias e covariancias, construir a análise de variancia e efetuar os testes $F$ de forma exata, quando os coeficientes $c_{i}^{\prime}$ ' são todos conhecidos, e em al gumas situaçбos mesmo, sendo parcial mente desconhecidos.

Procurou-se envolver no trabalho alguns modelos lineares, julgados ser bastante usuais nas aplicaçôes estatisticas. Alem disso, queremos crer que outros modelos, também muito usados como os de blocos aleatorizados, blocos incompletos, quadrados latinos e outros, possam ser abordados por metodologia análoga a que aqui se apresenta.

- caso de modelos com um rator fixo bal anceado foi tratado por SCARIANO e DAVENPORT (1887) e por PERES (1985), portanto não roi tratado no capítulo 3. Procedeu-se a uma generalização para o caso não balanceado. Como se pode ver, para planejamentos não balanceados. existem algumas dificuldades na forma de escrever as matrizes devidas as somas de quadrados dos parámetros, pois as mesmas envolvem, além do produto de Kronecher, as somas diretas; assim como na resolução de sistemas lineares. cuja finalidade e encontrar $c_{i}$ tal que $\Omega \in \mathbb{F}$.

o caso de equi correl ação tratado no item 3.2.2. pode ser visto da seguinte forma: se o experimento é bal anceado, então a equicorrelação é a usual, isto e, a matriz $\Omega$ é uma matriz de blocos diagonal uniforme, e em 
decorréncia do desbalanceamento, $\Omega$ sofre uma perturbaçăo nos blocos diagonais, onde as variáncias e covariancias dos blocos são perturbadas por um fator de proporcionalidade que depende dos tamanhos amostrais.

Para modelos com dois fatores, estudou-se apenas o caso balanceado, pois, alem das dificuldades de se trabalhar com modelos desbalanceados, nåo podemos construir a análise de variáncia da forma usual, uma vez que ela depende das várias formas de partiçăo da soma de quadrados, podendo não nos levar a testes $F$ exatos, mesmo quando os erros não são correlacionados, veja MILLER (1086 - cap. 4).

Pode-se notar que. para cada modelo. foi primeiramente estudado o caso de não independéncia dos erros, a fim de que se possa notar as diferenças que devem ocorrer por ocasiăo da considerada correlaçăo. Inicialmente foram considerados modelos com dois fatores fixos sem interaçăo, permitindo dois casos de equicorrelaçăo: somente dentro de caselas (quando $k$ > 1 ) e dentro dos niveis de um dos fatores. Se $k=1$, entao $\rho=0$ e a analise ó feita de maneira usual para o primeiro caso, e para o segundo caso. se $k=1$, o experimento pode ser visto como um experimento com dois fatores onde um deles corresponde a repetiçơs das observaçơes que são equicorrellacionadas.

Foram estudados a seguir os experimentos com dois fatores fixos com interaçăo $(K>1)$, permitindo trés casos de equicorrelação: somente dentro de caselas, dentro dos niveis de um dos fatores, e ainda um caso mais geral, 
onde se permite as correlaçன̆es entre observaçర̃es dentro de caselas. por um valor " $\rho_{1}$ "e entre observaçós dentro do mesmo nivel de um dos fatores, por " $P_{2}$ ". Para este terceiro caso, pode-se verificar que, quando $P_{1}=P_{2}$, este caso reduz-se ao segundo caso, e se $\rho_{2}=0$, reduz-se ao primeiro caso, ambos aqui tratados.

A seguir foram estudados os modelos mistos com interaçåo balanceados. Como foram consideradas as restriçరిes parambtricas usuais, foram também consideradas as covariancias entre os efeitos de dois tratamentos devido a interação e em niveis diferentes, o que não alterou em muito as regras da análise de variáncia pretendida. Foram tratados dois casos de equicorrelação: somente dentro de caselas e dentro dos niveis do fator fixo.

Verificoutse que em alguns casos de equicorrelaçăo, nem todas as hipóteses apresentam vies nas probabilidades dos erros tipos I e II. como por exemplo o caso (3.3.1.b.2.) para modelos com dois fatores fixos sem interação e equicorrelação entre obsevaçర̋es dentro de cada nivel do fator $\alpha$, e independência das observaçбes em niveis diferentes. Neste caso, a hipótese $H^{(2)}$. isto $\sigma_{0} H_{0} \beta_{j}=0$ $v=H_{1}: \beta_{j} \neq 0$ para algum $j=1,2, \ldots, s$, pode ser testada sem erro pelos mótodos usuais de análise de variancia. Nos modelos com interaçăo, ocorreu fato semelhante para esse mesmo caso de equicorrelação . sendo que alem de $H^{(2)}$. tem-se que $H^{(3)}$ também pode ser testada sem erro pelos metodos usuais da análise de variancia. 
Exceto esses casos citados, todos os demais testes $F$ usuais da análise de variáncia, para as demais hipóteses. apresentaram viéses quanto as probabilidades dos erros tipos I e II, sob a presença de correlação dos erros do modelo, com dois fatores fixos.

Nos modelos mistos notou-se que, para o primeiro caso de equicorrel açăo (3.4.1.b.1.). a hipótese $H^{(1)}$ para o fator fixo pode ser testada sem o comprometimento das probabilidades dos erros tipos I e II e para o segundo caso (3.4.1.b.2.). isso acontece para $H^{(2)}$ e $H^{(a)}$. isto é. só há o comprometimento das probabilidades dos erros para a hipótese $H^{(1)}$.

PERES (1885), quando mostra a influsncia das correlaçơes na análise de variancia com um fator fixo e balanceado: propơe também uma solução alternativa para superar esse problema, através de repetiçбes experimentais que acarretam uma modificaçăo no modelo em questão, com a inserção do fator repetiçăo (aleatóriol a esse modelo. Assim, o autor resolve seu problema, sem que haja necessidade de conhecer o valor da correlaçăo em questão. $€$ natural que nem sempre o pesquisador tenha disponibilidade de planejar seu experimento com repetiçరes. Em outros casos. pode ocorrer que, só dépois de realizar o experimento.o pesquisador depare com a presença de correlaçర̃es nos seus dados, e se não houver jeito de dividir seu dados de forma a considerá-los como repetiçơes independentes, então ele deve procurar resolver seu problema de outra forma que não essa. 
Caso o experimentador conheça o valor da correlaço om questá ontzo elo pode razer uso da teoria que transforma um modelo linear geral de Gauss-Markov para um modelo ordinário, ou equivalentemente utilizar um procedimento análogo àquele dado por (3.2.1.c.1.).

Neste trabalho. buscou-se um procedimento alternativo, na mesma linha de PERES (1985), para todos os modelos e casos de equicorrelação aqui abordados. Geralmente,o fator repetição inserido no modelo a aleatório e hierárquico a um dos fatores em questão, ou a interação, e com distribuição normal independente da distribuição dos erros e dos demais fatores cno caso de modelos mistos ou aleatórios). Nesse contexto, deve-se cuidar para que a inclusão do fator repetição não comprometa sua correspondente matriz responsável pela soma de quadrados, que deve ser projetor ortogonal, assim como as demais matrizes das qutras somas de quadrados envolvidas. Deve-se determinar, também, a matriz de variancias e covariancias do atual modelo, de modo que esta pertença a classe $\mathfrak{Z}$ ou verificar se dessa forma pode-se montar um sistema de equaçres linearmente independentes que nos permite encontrar os valores de $c_{i} e$, finalmente, verificar se a nova analise de variancia (univariada) permite todos os testes $F$ exatos para as hipóteses principais. Caso algum desses passos não esteja satisfeito, pode-se tentar novamente com a inclusão do fator repetição, de forma diferente. Para alguns modelos e casos de equicorrelação. 
não foi possivel a solução alternativa e portanto eles não constam deste trabalho.

Para todos os casos pode-se notar que, mesmo para baixas correlaçơes, o viés já comprometedor e aumenta a medida que essas correl açóes aumentam.

No modelo aleatório com um fator, aqui tratado no parágrafo 3.2.1.. pode-se notar que na TABELA A.1. do apendice, para probabilidades dos erros tipo I. à medida que os tamanhos amostrais crescem assim como o valor de $\rho$. - viés aumenta no sentido positivo para $\rho>0$ e negativo para $\rho$ <. Nota-se ainda que para correlaçðes positivas esse viés é maior, ou seja, o caso se torna mais grave. Quanto a TABELA A.2., que fornece as probabilidades dos erros tipo II. verifica-se que o viés ocorre no sentido contrário ao viés da TABELA A.1.. e que para pequenas correlaçôes esse viés é praticamente o mesmo para $\rho>0$ e $\rho<0$, mas, conforme $\rho$ aumenta, torna-se maior para $\rho>0$. Aqui também nota-se que o problema se agrava a medida que os tamanhos amostrais aumentam, assim como o número de níveia dos fatores. Na TABELA A. 2. toma-se $\left(\sigma_{\alpha}^{2} / \sigma_{e}^{2}\right)=1$ como hipótese alternativa, isto é $\alpha_{\alpha}^{2}=\sigma_{\theta}^{2}$, e de uma rápida análise da construção dessas probabilidades pode-se notar que essé viés tende a aumentar se $\alpha_{\alpha}^{2}<\sigma_{e}^{2}$ e a diminuir se $\alpha_{\alpha}^{2}>\alpha_{e}^{2}$.

No modelo de efeitos fixos e não balanceado. aqui tratado, pode-se ver que as probabilidades dos erros tipos I e II não dependem diretamente do desbalanceamento. 
mas se esse desbalanceamento for muito acentuado, e como a matriz de variancias e covariancias deve ser positiva definida, o valor de " $\rho$ " pode extrapolar o intervalo real [-1 , 1 ]. invalidando assim a estrutura aqui imposta. Pode-se ver que o viés aumenta rapidamente, à medida que o número de niveis do fator aumenta,mantendo-se o mesmo número total de observaçðes e mesma correlaçăo $\rho$. Acontece o mesmo se mantivermos fixo o número de niveis do fator e de $\rho$ e aumentar o número total de observaçóes. Aqui também ocorre que para $\rho>0$, o viés é maior do que para $\rho<0$ nos dois tipos de erros. Para as probabilidades dos erros tipo II. ainda observa-se que conforme 0 parametro de não centralidade $\lambda$ aumenta, as probabilidades diminuem. Isto ocorre pelo maior achatamento da curva $F$ não central, quanto maior o seu parámetro de não centralidade.

No modelo com dois fatores $\mathrm{fixos}$ sem interação tem-se dois casos de equicorrelaçăo. o primeiro caso 3.3.1.b.1.2, cujas probabilidades são dadas pelas TABELAS A. 5. e A.6. do apéndice, para $\rho \geq 0$, pois para esse caso não podemos ter $\rho<0$. Nota-se que as probabilidades do erro tipo I crescem rapidamente para a unidade, ao contrário das probabilidades do erro tipo II. 0 viés aumenta com o número de observaçőes dentro de caselas, assim como para - número de níveis dentro do fator, correspondente à hipótese de interesse. Quando esse aumento do número de niveis ocorre no outro fator e nao naquele da hipótese de 
interesse, o viés é menor. Podemos notar que para $\lambda=20$ as probabilidades dos erros tipo II são muito pequenas quando comparadas com as probabilidades para os casos am que $\lambda=5$. Isto indica que o achatamento da curva $F$ não central. para esse modelo, é mais acentuado do que para o modelo de um fator fixo. Como no segundo caso (3.3.1.b.2.), so ocorre viés nas probabilidades dos erros para $H^{(1)}$, e mesmo assim, com valores iguais àqueles do caso (3.3.1.b.1.) segundo os mesmos $\rho^{\prime} s$, as observaçช̆es são análogas, salvo que, aqui permite-se que $\rho$ assuma valores negativos, (num pequeno intervalo).

Para modelos com dois ratores fixos interação, foram abordados 3 casos de equicorrelaçăo, a saber: caso (3.3.2.b.1.), que supōe equicorrelação somente dentro de caselas; caso (3.3.2.b.2.). que supre equicorrelação entre as observaçơes no mesmo nivel de um dos fatores; e o caso (3.3.2.b.3.), que engloba os dois casos anteriores, isto é, as observaçóes dentro da mesma casela são equicorrelacionadas por $\rho_{1}$ e fora das caselas, mas no mesmo nivel de um dos fatores, são equicorrelacionadas por $\mathrm{P}_{2}$.

No caso (3.3.2.b.1.), nota-se que, mesmo para pequenos valores de $\rho$, o viés nas probabilidades é considerável, sendo que à medida em que os tamanhos amostrais aumentam assim como os valores de $\rho$, o vies das probabilidades dos erros tipo I tamberm crescem, e crescem mais quando $\rho$ tende para o valor 1 . Para os mesmos valores 
de r. s o k o vies o mesmo para $H^{(1)}$ o $H^{(2)}$ o malor para $H^{(a)}$. Para as probabilidades dos erros tipo II, além das observaçöes relatadas, pode-se ver que para $\lambda=5$ e $\lambda=$ 20 o viés é maior para valores negativos de $\rho$ e próximos de zero; e com o crescimento dos tamanhos amostrais assim como de $\rho$, essa situação tende a inverter-se. Nota-se ainda que quando $\lambda$ aumenta, a probabilidade do erro tipo II, em si, diminui, mas o viés aumenta e isto se agrava com o aumento de $\rho$ e dos tamanhos amostrais.

Para o caso (3.3.2.b.2.) temos viés somente na hipotese $H^{(1)}$, mas o intervalo permitido para $\rho$ é menor a esquerda. Novamente observa-se que, para valores positivos de $\rho$, o viés é maior para a probabilidade dos erros tipo I (TABELA A.11), e menor para as probabilidades dos erros tipo II ( TABELA A.12).

Neste caso, nåo fica claro que, conforme o aumento de $\lambda$, se verifica o crescimento do viés das probabilidades.

Para o caso (3.3.2.b.3.) considera-se trés valores de $P_{2}$, a saber, $P_{2}=0.1, P_{2}=0.3 e P_{2}=0.5$. com sete valores de $\rho_{1}$ para cada valor de $\rho_{2}$. Os valores de $\rho_{1}$ não são os mesmos para cada $\rho_{2}$, pois dependem do valor de $\rho_{2}$, isso no cálculo do viés das probabilidades do erro tipo I; para as probabilidades do erro tipo II considerou-se $\rho_{2}=0.3$.

Nota-se na TABELA A.13. que, mantendo-se fixo - valor de $\rho_{2}$, o viés das probabilidades dos erros tipos I 
tendem para 1 . d medida em que ou $\rho_{1}$ ou os tamanhos amostrais crescem.

Por outro lado, mantendo-se $r i x o$ o valor de $P_{i}$ e fazendo-se $\rho_{2}$ crescer, não se caracteriza um crescimento dessas probabilidades. Confrontando as TABELAS A. 8. e A.13. do apéndice, dos casos (3.3.2.b.1.) e (3.3.2.b.3.), respectivamente, observa-se que o viés é maior para o teste de $H^{(1)}$. e menor para os testes de $H^{(2)}$ e $H^{(3)}$, sob a presença da correlação $\rho_{2}>0$.

O comportamento das probabilidades da TABELA A. 14. para $\lambda=5$ e $\lambda=20 \Leftrightarrow$ semelhante ao da TABELA A.10. no sentido de que as probabilidades decrescem rapidamente para zero, quando ou $\rho_{i}$ ou os tamanhos amostrais crescem, assim como crescem para a unidade quando $P_{1}$ decresce. Nota-se que a presença da correlação $P_{2}$ modifica consideravelmente as probabilidades dos erros tipo II. cuja diferença é menor para grandes valores de $P_{1}$. Ainda nas tabelas das probabilidades dos erros tipo II. para $\lambda \neq 0$, pode-se notar grandes diferenças desses valores da hipotese $H^{(1)}$ em relaçăo as hipoteses $H^{(2)} \ominus H^{(a)}$, no sentido de que tais probabilidades para $H^{(1)}$ săo bem menores que para $H^{(2)} \mathrm{H}^{(3)}$, amenizando tal situação para grandes valores de $P_{1}$.

De uma análise geral deste trabalho pode-se observar que essas Tabelas poderiam ser produzidas para casos mais gerais de correl açôes e também segundo o modelo, desde que a matriz $\Omega$ seja tal que $\Omega \in \mathcal{Z}$. 


\section{CONCLUSŌES}

Considerando-se os objetivos deste trabalho,e com base nos tópicos desenvolvidos e nas tabelas aqui obtidas para esses modelos, pode-se concluir que:

5.1) A presença de correlaçơes, ainda que pequenas, podem comprometer seriamente os testes $F$ da análise de variancia usual, ou parte deles, no que diz respeito ao nivel de significancia e poder dos testes.

5.2) Pode-se observar pelos resultados obtidos que, nestes casos de modelos com distribuiçós multinormais, quanto maior o tamanho amostral ou número de niveis dos fatores, maior será o viés nas probabilidades dos erros tipos I e II. 5. 3) Os testes $F$ da análise de variancia usual, para as hipóteses principais, não são igualmente afetados pelas correlações. Dependendo do caso de equicorrelação considerado, os viéses nas probabilidades dos erros tipos I e II sao, em geral, diferentes para as diferentes hipóteses, podendo mesmo ser nulos para uma ou mais delas. 5.4) Das tabelas das probabilidades dos erros tipo I. nota-se que, para valores positivos de " $\rho$ ", o comprometimento 
do nivel de significáncia é maior do que para valores negati vos.

5.5) Nota-se que, segundo a metodologia aqui empregada. o limite inferior de " $\rho$ " depende do caso de equicorrelação do modelo e do tamanho amostral considerado. Isso não acontece para o limite superior, que sempre a unidade.

5.6) O conhecimento prévio da presença de correlaçóes nos dados pode levar o pesquisador a planejar o seu experimento, de forma que ele possa efetuar a usual análise de variancia e realizar os testes das hipóteses principais sem erro. Não temos o conhecimento da existência de uma regra básica que permita resolver esse problema para todos os modelos. Mas, de acordo com o desenvolvimento deste trabalho, esse problema pode ser contornado para alguns modelos e alguns casos de equicorrelação, "através de repetiçชes independentes do experimento.

5.7) Nota-se que, sob a hipótese alternativa, as probabilidades dos erros tipo II decrescem à medida em que ou $\lambda$ ou $\rho$ crescem, isto $e$, o poder do teste aumenta a medida que se afasta da hipótese nula $H_{0}$ e também aumenta a medida que $\rho$ cresce $C$ mas aqui com prejuizo do nivel de significáncia .

Pode-se ainda concluir que esse trabalho nos permitiu tratar a análise de variancia de uma forma simples, mesmo na presença de correlaçőes dos dados; assim como o método numérico para obtenção das probabilidades dos erros tipo II, com a ajuda de recursos computacionais. 


\section{REFERÊNCIAS BIBLIOGRAF́ICAS}

ANDERSEN, A.H. ; JENSEN, E.B. ; SCHOW, G. ; 1981 . TwO-WaY Analysis of Variance with Correlated errors; International Statistical Review; London, 49: 153-167.

ANDERSON, V.L. \& MCLEAN, R.A.; 1874. Another Dimension in Teaching Experimental Statistics; The American Statistician, Washington, 28( 4):145-152.

BOX, G.E.P.;1949. A General Distribuition Theory For a Class of Likelihood Criteria; Biometrica, Raleigh, 36: $317-346$.

; 1950. Problems in the analysis of growth and wear curves; Biometrics 6:362-

1954(a). Some Theorems on Quadratic Forms Applied in the Study of Analysis of Variance Problems, I. Efrect of Inequality of Variance in the One-Way Classification; Annals Math. Statist. : Baltimore, 25: $290-302$. 
; $1854(b)$. Some Theorems on Quadratic Forms Applied in the Study of Analysis of Variance Problems. II. Effect of Inequality of Variance and of Correlation Between Errors in The Two-Way Classification; Annals. Math. Statist., Baltimore, 25: 484-498.

; HUNTER, W.G.; HUNTER, J.S. ; 1978. Statistics

For Experimenters - An Introduction to Design Data Analysis, and Model Building. Wiley, N.Y., 653 p.

DANFORD, M.B.; HUGUES, H.M.; MCNEE, R.C.; 1860 . On the Analysis of Repeated-Measurements Experiments; Biometrics, Raleigh, 16: 547-565.

FOX. M.; 1956. Charts of Power of the F-Test. Annals Math. Stat., Baltimore, 27( 42) : 484-497.

GILL, J.L. \& HAFS, H.D.; 1871. Analysis of Repeated Measurements of Animals; Journal of Animal Science, 33(2) : $331-336$.

GILL,P.S. \& SHUKLA, G.K.; 1985-a. Experimental Designs and Their Efficiencies for Spatially Correlated Observations in Two Dimensions. Commun. Statist. Theor. Meth., $14(9): 2181-2197$.

; 1885-b. Efficiency of Nereast Neighbour Balanced Block Designs for Correlated Observations; Biometrika, Raleigh, 72( 3$): 539-544$. 
GRAYBILL, F.A.; 1961. An Introduction to Linear Statistical Models, V.I.. McGraw-Hill, N.Y. 463 p.

HENDERSON, R. ; 1986. Change-Point Problem With Correlated Observations, With an Application in Material Accountancy, Technometrics, Al exandria, 28( 4): $381-389$.

HUYNH, H. \& FELDT, L.S. ; 1970 . Conditions Under whith Mean Square Rations in Repeated Measurements Desigs Have F-Ditribuitions; Journal of the American Statistical Association, Washigton; 65(332): $1582-1589$.

IEMMA, A.F.; 1981. Analise de Experimentos em Parcelas Subdivididas com Tratamentos Principais Dispostos em Blocos Incompletos Balanceados. Piracicaba, 145 p. CTese de Doutoramento - ESALQ/USPS.

; 1982. Valores Singulares de Matrizes Uniformes. Ciencia e Natura, Santa Maria, R.S. 4 : $21-26$. ; 1983. Testes de Hipóteses nos Experimentos com Parcelas Subdivididas em Blocos Incompletos Balanceados. Desarrollo Rural en Las Americas, Costa Rica, 2 : 143-151.

- 1985. Modelos Lineares para Experimentos com Medidas Repetidas: Um Enfoque Através das Matrizes de Helmert. Piracicaba, 155 p. (Livre-docéncia - ESALQ USP). 
; 1987. Modelos Lineares; Uma Introdução para

Profissionais da Pesquisa Agropecuária. Londrina, UEL Dopartamento de Matemática Aplicada, $253 \mathrm{p}$.

para Profissionais de Ciéncias Aplicadas - ESALQ - USP. $339 \mathrm{p}$.

KENNY, D. A. \& JUDD, C.M.; 1986. Consequences of Violating the Independence Assumption in Analysis of Variance. Psichological Bulletin, 99(3):422-431.

MARTIN, R.J. ; 1982 . Some Aspects of Experimental Design and Analysis when Errors Are Correlated. Biometrika, Raleigh, $\underline{69}(3): 597-612$.

MILLER, Jr., R.G.; 1986. Beyond ANOVA Basics of Applied Statistics. Wiley, N.Y., $317 \mathrm{p}$.

PEARSON, K.; 1934. Tables of the Incomplete Beta Function. Cambridge University Press, Cambridge.

PEARSON, E.S. \& HARTLEY, H.O.; 1951. Charts of the Power Funtion of Analysis of Variance Tests, Derived from the Non-Central F-Distribution. Biometrika, Raleigh, 38(41):112-130. 
PERES, C.A.; 1985. Análise de Variáncia sob a Presença de Correlação entre os Erros. XII Semana de La Matemática. Univ. Cat. de Val Paraiso - Inst. de Matematicas, Argentina, 11 p.

PRAETZ, P.; 1981. A Note on the Effect of Auto Correlation on Multiple Regression Statistics; Austral. J. Statist.. $23(3): 309-313$.

PRESS,H.W.; FLANNERY, B.P.; TEUKOLSKY, S.A. \& VETTERLING, W. I.; 1986. Numerical Recipes. Cambridge University Press, Cambridge. 800 p.

REILMAN, M. A. ; GUNST, R.F. $\varepsilon$ LAKSHAMIMARAYANAN, M. Y.; 1985. Strutural Model Estimation with Correlated Measurement Errors, Biometrika, Raleigh, 72(3):669-672.

ROGERS, G.S.; 1984. Kronecher Products in ANOVA - A First Step; The American Statistician, Washigton, 38(3):197-202.

SCARIANO, M.S. \& DAVENPORT, J.M.; 1987. The Efrets of Violations of Independence Assumptions in the One-Way ANOVA. The American Statistician, Washigton, $41(2)$ : $123-129$

SCHEFFE, H.; 1959. The Analysis of Variance. Wiley, M.Y., $477 \mathrm{p}$.

SEARLE, S.R.; 1971. Linear Models. Wiley, N.Y. 532 p. 
; 1987. Linear Models for Unbalanced Data.

Willey, N.Y., 534 P.

SINGER, J.M. \& ANDRADE, D.F.; 1986. Análise de Dados Longitudinais, VII. Simpósio Nacional de Probabilidade Estatística, Campinas, $106 \mathrm{p}$.

TANG, P.C.; 1938. Power of the F-Test in Terms of Non-Central F-Distribution, Statist. Res. Mem.. 2: $126-149$.

TIKU, M.L.; 1964. Approximating the General Non-Normal Variance-Ratio Sampling Distributions, Biometrika, Raleigh, 51: 83-95.

; 1965. Laguerre Series Forms of Non-Central $x^{2} e$ F Distributions, Biometrika. Raleigh, 52(3 e 4):415-427.

; 1967. Tables of The Power of The F-Test; Journal of the American Statistical Association,

Washington, $62(318): 525-539$.

ZIMMERMANN, F.J.P.; 1987. Efeito de Heterogenei dade de Variancia e Distribuição de Probabilidade dos Dados sobre - Poder e Tamanho do Teste F, Pesq. Agropec. Bras.. Brasilia, 22(11/12):1209-1213. 
WILKS, S.S. ; 1946. Sample Criterion for Testing Equality of Moans, Equality of Variances and Equality of Covariances in a Normal Mulvariate Distribution; Ann. Math. Stat., Baltimore, 17: 257-281. 


$$
A P \hat{E} N D I C E
$$


TABEla A.1. Frobabilidades do erro tipo I, para a hiputese $H_{0}$, ao nivel nominal de significáncia 0,05 , segundo os varios valores de $r, k e p$.

\begin{tabular}{|c|c|c|c|c|c|c|c|c|c|c|c|}
\hline & \multicolumn{10}{|c|}{$p$} \\
\hline$r$ & $\mathrm{k}$ & - & 9000 & - & .5000 & - & 1000 & .0000 & .1000 & .5000 & .9000 \\
\hline \multirow[t]{4}{*}{2} & 2 & & .0028 & & .0175 & & .0415 & .0500 & .0601 & .1310 & .4277 \\
\hline & 3 & & - & & - & & .0312 & .0500 & .0740 & .2373 & .6275 \\
\hline & 5 & & - & & - & & 0142 & .0500 & .1016 & .3739 & .7475 \\
\hline & 10 & & - & & - & & 0000 & .0500 & .1656 & .5346 & .8282 \\
\hline \multirow[t]{4}{*}{3} & 2 & & .0007 & & 0111 & & 0384 & .0500 & .0646 & .1813 & .6483 \\
\hline & 3 & & - & & - & & 0265 & .0500 & .0838 & 3432 & .8368 \\
\hline & 5 & & - & & - & & 0091 & .0500 & .1236 & .5403 & 9184 \\
\hline & 10 & & - & & - & & 0000 & .0500 & .2231 & .7400 & .9839 \\
\hline \multirow[t]{4}{*}{5} & 2 & & 0001 & & .0050 & & 0340 & .0500 & .0723 & .2793 & .8836 \\
\hline & 3 & & - & & - & & 0204 & .0500 & .0986 & .5147 & 9704 \\
\hline & 5 & & - & & - & & 0046 & .0500 & .1598 & .7513 & 9922 \\
\hline & 10 & & - & & - & & 0000 & .0500 & 3129 & .9169 & 9984 \\
\hline \multirow[t]{4}{*}{10} & 2 & & .0000 & & 0010 & & 0270 & .0500 & .0875 & .4912 & 9846 \\
\hline & 3 & & - & & - & & 0128 & .0500 & 1328 & .7843 & 9997 \\
\hline & 5 & & - & & - & & 0013 & .0500 & 2372 & .9502 & 1.0000 \\
\hline & 10 & & - & & - & & 0000 & .0500 & .4967 & .9957 & 1.0000 \\
\hline
\end{tabular}

TABELA A.2. Probabilidades do erro tipo II, para a hipótese $H_{0}$, ao nivel nominal de significancia 0,05 , segundo os vários valores de $r, k \in \rho$ para $\alpha_{\alpha}^{2}, \alpha_{e}^{2}=1$.

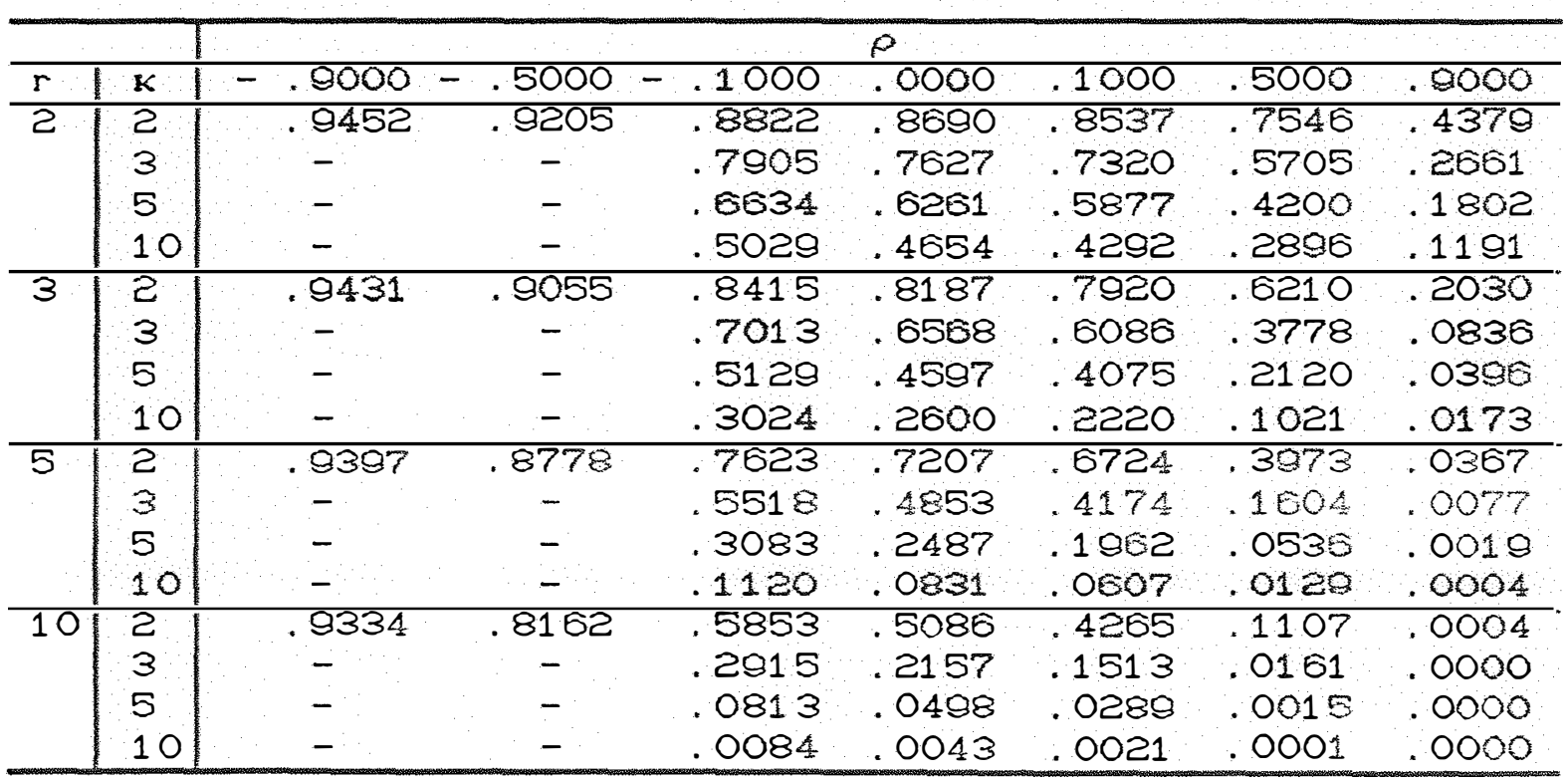


TABELA A. 3. Probabilidades do erro tipo I, para a hipótese $H_{0}$ ao nivel nominal de significancia 0,05 , segundo os vários valores de $N, r$ e $p$ para $p=\alpha_{e}^{2}$ e $q=p \alpha_{e}^{2}$.

\begin{tabular}{|c|c|c|c|c|c|c|c|c|c|c|c|}
\hline & \multicolumn{10}{|c|}{$\bar{\rho}$} \\
\hline$N$ & $r$ & - & .9000 & - & .5000 & - & .1000 & .0000 & .1000 & .5000 & .9000 \\
\hline \multirow[t]{2}{*}{6} & 2 & & - & & - & & .0312 & .0500 & .0740 & .2373 & .6275 \\
\hline & 3 & & .0007 & & .0111 & & .0384 & .0500 & .0646 & .1813 & .6483 \\
\hline \multirow[t]{3}{*}{10} & $\hat{2}$ & & - & & - & & .0142 & .0500 & .1016 & .3739 & .7475 \\
\hline & 3 & & - & & - & & . O229 & .0500 & .0902 & .3860 & .8610 \\
\hline & 5 & & 0001 & & .0050 & & .0340 & .0500 & .0723 & .2793 & 8836 \\
\hline \multirow[t]{3}{*}{20} & 2 & & - & & - & & .0000 & .0500 & .1656 & .5346 & .8282 \\
\hline & 3 & & - & & - & & .0020 & .0500 & .1578 & 6339 & 8430 \\
\hline & 5 & & - & & - & & 0107 & .0500 & .0488 & 1288 & .8865 \\
\hline \multirow[t]{4}{*}{30} & 2 & & - & & - & & - & .0500 & .2189 & .6124 & .8617 \\
\hline & 3 & & - & & - & & .0000 & .0500 & .2231 & .7400 & .9639 \\
\hline & 5 & & - & & - & & .0015 & .0500 & .1817 & .8107 & .9950 \\
\hline & 10 & & - & & - & & .0128 & .0500 & .1328 & .7843 & .9997 \\
\hline \multirow[t]{4}{*}{50} & 2 & & - & & - & & - & .0500 & .3063 & .6952 & .8941 \\
\hline & 3 & & - & & - & & - & .0500 & .3353 & .8353 & .8791 \\
\hline & 5 & & - & & - & & .0000 & .0500 & .3148 & .9175 & .9984 \\
\hline & 10 & & - & & - & & .0013 & .0500 & .2372 & .9502 & 1.0000 \\
\hline \multirow[t]{4}{*}{90} & 2 & & - & & - & & - & .0500 & .4199 & .7704 & .9218 \\
\hline & 3 & & - & & - & & - & .0500 & 4919 & 9049 & 9887 \\
\hline & 5 & & - & & - & & - & .0500 & .5118 & .9709 & 9995 \\
\hline & 10 & & - & & - & & .0000 & .0500 & .4550 & .9938 & 1.0000 \\
\hline
\end{tabular}


TABELA A. 4. Probabilidades do erro tipo II, para a hipotese Ho, a nivel nominal do significkncia: 0,05 , sogurio os vários valores deN, $F, P, \lambda=0,5 \theta 20$.

\begin{tabular}{|c|c|c|c|c|c|c|c|c|c|c|}
\hline \multicolumn{2}{|c|}{$\lambda=0$} & \multicolumn{9}{|c|}{$p$} \\
\hline$N$ & $r$ & - & .9000 & - & .5000 & -.1000 & .0000 & .1000 & .5000 & .900 \\
\hline \multirow[t]{2}{*}{6} & 2 & & - & & - & .9688 & .9500 & .9260 & .7627 & .3725 \\
\hline & 3 & & .9993 & & .9889 & .9616 & .9500 & .9354 & .8187 & .3517 \\
\hline \multirow[t]{3}{*}{10} & 2 & & - & & - & .9858 & .9500 & .8984 & .6261 & .2575 \\
\hline & 3 & & - & & - & 9771 & .9500 & .9098 & .6140 & 1319 \\
\hline & 5 & & .8989 & & .9950 & .8660 & .9500 & .9277 & .7207 & .1154 \\
\hline \multirow[t]{3}{*}{20} & 2 & & - & & - & 1.0000 & .9500 & .8344 & .4654 & .1718 \\
\hline & 3 & & - & & - & .9980 & .9500 & .8422 & .3661 & .0570 \\
\hline & 5 & & - & & - & .9893 & .9500 & .8711 & .3396 & .0135 \\
\hline \multirow[t]{4}{*}{30} & 2 & & - & & - & - & .9500 & .7801 & .3876 & .1383 \\
\hline & 3 & & - & & - & 1.0000 & .9500 & .7769 & 2600 & 0301 \\
\hline & 5 & & - & & - & .9985 & 9500 & .8083 & .1893 & .0050 \\
\hline & 10 & & - & & - & .9872 & 9500 & .8672 & .2157 & .0003 \\
\hline \multirow[t]{4}{*}{50} & 2 & & - & & - & - & .9500 & .6937 & .3048 & .1059 \\
\hline & 3 & & - & & - & - & .9500 & .6647 & .1647 & .0200 \\
\hline & 5 & & - & & - & 1.0000 & 9500 & .6852 & .0825 & .0016 \\
\hline & 10 & & - & & - & .9987 & .9500 & .7628 & .0498 & .0000 \\
\hline \multirow[t]{4}{*}{90} & 2 & & - & & - & - & .9500 & .5801 & .2296 & $.0782^{3}$ \\
\hline & 3 & & - & & - & - & .9500 & .5081 & .0951 & .0113 \\
\hline & 5 & & - & & - & - & 9500 & .4882 & .0291 & .0005 \\
\hline & 10 & & - & & - & 1.0000 & .9500 & .5450 & .0062 & .0000 \\
\hline \multicolumn{2}{|c|}{$x=5$} & \multicolumn{9}{|c|}{$p$} \\
\hline$N$ & $r$ & - & 9000 & - & .5000 & -.1000 & .0000 & .1000 & .5000 & .9000 \\
\hline \multirow[t]{2}{*}{6} & 2 & & - & & - & .7012 & .5972 & .4982 & .1975 & .0521 \\
\hline & 3 & & 9958 & & .9420 & .8239 & .7807 & .7306 & .4459 & .0601 \\
\hline \multirow[t]{3}{*}{10} & 2 & & - & & - & .7322 & .4939 & .3363 & .0991 & .0421 \\
\hline & 3 & & - & & - & .7880 & .6532 & .5201 & .1521 & .0252 \\
\hline & 5 & & .9996 & & .9743 & .8611 & 8099 & .7470 & .3772 & . 0214 \\
\hline \multirow[t]{3}{*}{20} & 2 & & - & & - & .9992 & .4315 & .2069 & .0607 & .0387 \\
\hline & 3 & & - & & - & .9278 & .5653 & . 3191 & .0570 & .0188 \\
\hline & 5 & & - & & - & .8884 & .7026 & .5021 & .0736 & .0048 \\
\hline \multirow[t]{4}{*}{30} & 2 & & - & & - & - & .4144 & .1566 & .0515 & .0379 \\
\hline & 3 & & - & & - & 1.0000 & .5388 & .2317 & .0393 & .0178 \\
\hline & 5 & & - & & - & .9625 & .6676 & .3742 & .0330 & .0036 \\
\hline & 10 & & - & & - & .9271 & .8020 & .6225 & .0613 & .0001 \\
\hline \multirow[t]{4}{*}{50} & 2 & & - & & - & - & .4005 & .1109 & .0450 & .0373 \\
\hline & 3 & & - & & - & - & $.51 v_{1}$ & .1484 & .0283 & .0171 \\
\hline & 5 & & - & & - & 1.0000 & .6411 & .2344 & .0147 & .0030 \\
\hline & 10 & & - & & - & .9817 & .7674 & .4327 & .0096 & .0000 \\
\hline \multirow[t]{4}{*}{90} & 2 & & - & & - & - & .3913 & .0781 & .0411 & .0369 \\
\hline & 3 & & - & & - & - & .5072 & .0866 & .0224 & .0167 \\
\hline & 5 & & - & & - & - & .6241 & .1171 & .0074 & .0027 \\
\hline & 10 & & - & & - & 1.0000 & .7394 & .2172 & $.001 \geq$ & .0000 \\
\hline
\end{tabular}


TABELA A.4. Contiruaçäo para $\lambda=20$.

\begin{tabular}{|c|c|c|c|c|c|c|c|c|c|c|}
\hline \multicolumn{2}{|c|}{$\lambda=20$} & \multicolumn{9}{|c|}{$\rho$} \\
\hline$N$ & $r$ & - & .9000 & - & .5000 & -.1000 & .0000 & .1000 & .5000 & .9000 \\
\hline \multirow[t]{2}{*}{5} & 2 & & - & & - & .1721 & .0912 & .0468 & 0025 & .0001 \\
\hline & 3 & & .9795 & & .7663 & 4520 & .3703 & .2016 & .0568 & .0004 \\
\hline \multirow[t]{3}{*}{10} & 2 & & - & & - & .1265 & .0272 & .0081 & .0005 & .0001 \\
\hline & 3 & & - & & - & 2267 & .1040 & .0461 & .0017 & .0001 \\
\hline & 5 & & 9970 & & 8497 & .4833 & .3805 & .2836 & .0363 & .0001 \\
\hline \multirow[t]{3}{*}{201} & 2 & & - & & - & 9441 & .0126 & .0021 & .0003 & .0002 \\
\hline & 3 & & - & & - & 3765 & .0374 & .0073 & .0003 & .0001 \\
\hline & 5 & & - & & - & .3453 & .1189 & .0390 & .0007 & .0000 \\
\hline \multirow[t]{4}{*}{30} & 2 & & - & & - & - & .0103 & .0013 & .0003 & .0002 \\
\hline & 3 & & - & & - & 9957 & .0273 & .0033 & .0002 & .0001 \\
\hline & 5 & & - & & - & 5244 & .0787 & .0144 & .0002 & .0000 \\
\hline & 10 & & - & & - & 5186 & .2679 & .1152 & .0011 & .0000 \\
\hline \multirow{4}{*}{501} & 2 & & - & & - & - & .0089 & .0008 & .0003 & .0002 \\
\hline & 3 & & - & & - & - & .0217 & .0015 & .0002 & .0001 \\
\hline & 5 & & - & & - & 1.0000 & .0572 & .0045 & .0001 & .0000 \\
\hline & 10 & & - & & - & .6935 & .1773 & .0332 & .0001 & .0000 \\
\hline \multirow[t]{4}{*}{801} & 2 & & - & & - & - & .0081 & .0006 & .0003 & .0003 \\
\hline & 3 & & - & & - & - & .0189 & 0007 & .0002 & .0001 \\
\hline & 5 & & - & & - & - & .0467 & .0013 & .0001 & .0000 \\
\hline & 10 & & - & & - & 9999 & .1308 & .0066 & .0000 & .0000 \\
\hline
\end{tabular}


TABELA A.5. Probabilidades do erro tipo I, para as hipóteses $H^{\langle\{\rangle} e H^{\langle 2\rangle}$, ao nivel nominal de significancia 0,05 segundo os vários valores de $r, s, k \in p$.

\begin{tabular}{|c|c|c|c|c|c|c|c|c|c|c|}
\hline \multirow[b]{2}{*}{$r$} & \multirow[b]{2}{*}{ Is } & \multirow[b]{2}{*}{ K } & \multirow[b]{2}{*}{$\mathrm{H}^{*}$} & \multicolumn{6}{|c|}{$p$} & \multirow[b]{2}{*}{.9000} \\
\hline & & & & .0000 & .0500 & .1000 & .2000 & .5000 & .7000 & \\
\hline \multirow[t]{6}{*}{$\overline{2}$} & 13 & 3 & 11 & .0500 & - & - & - & 5087 & .6545 & .8162 \\
\hline & & & 2 & .4500 & - & - & - & .5976 & .7827 & .9346 \\
\hline & & 5 & 1 & .0500 & - & - & .3540 & .6115 & .7345 & .8614 \\
\hline & & & 2 & .0500 & - & - & .3950 & .7387 & 8713 & 9637 \\
\hline & & 10 & 1 & .0500 & - & .3408 & .4955 & .7208 & .8133 & $90 \geqslant 6$ \\
\hline & & & 2 & .0500 & - & .3825 & .5944 & .8611 & .9381 & 9828 \\
\hline \multirow[t]{6}{*}{$\overline{2}$} & 5 & 3 & 1 & .0500 & - & $=$ & .3547 & .6118 & .7347 & .8615 \\
\hline & & & 2 & .0500 & - & - & .3811 & .8127 & 9451 & 9950 \\
\hline & & 5 & 1 & .0500 & - & 3055 & .4582 & 6946 & 7949 & 8939 \\
\hline & & & 2 & .0500 & - & .3133 & .5698 & 9168 & .9797 & 9984 \\
\hline & & 10 & 1 & .0500 & 3009 & .4407 & .5908 & .7819 & .8554 & 9259 \\
\hline & & & 2 & .0500 & .3167 & .5517 & .8010 & 9762 & .9949 & 9806 \\
\hline \multirow[t]{6}{*}{5} & 3 & 2 & 1 & .0500 & - & .1889 & .3714 & .8066 & .9429 & .8948 \\
\hline & & & 2 & .0500 & - & .2197 & .3915 & .7358 & .8697 & .9632 \\
\hline & & 5 & 1 & .0500 & . 2388 & .4414 & .7122 & .9587 & .9908 & .9993 \\
\hline & & & 2 & .0500 & .2635 & .4388 & .6494 & .8860 & .9483 & .9862 \\
\hline & & 10 & 1 & .0500 & 4547 & .7016 & .8913 & .9893 & .9978 & 9998 \\
\hline & & & 2 & .0500 & .4398 & .6337 & .8010 & .9429 & .9748 & 9933 \\
\hline \multirow[t]{6}{*}{5} & 5 & 2 & 1 & .0500 & .1663 & .3106 & 5665 & .9157 & .9793 & .9983 \\
\hline & & & 2 & .0500 & .1603 & .3106 & .5665 & 9167 & 9793 & .9983 \\
\hline & & 5 & 1 & .0500 & .3806 & .6291 & .8519 & 9841 & 9967 & 9998 \\
\hline & & & 2 & .0500 & .3806 & .6291 & .8519 & 9841 & 9987 & 9998 \\
\hline & & 10 & 1 & .0500 & .6256 & .8359 & $.95 \bullet 8$ & .9959 & 9992 & 9999 \\
\hline & & & 2 & .0500 & .6256 & .8359 & .9508 & 9959 & .9992 & .8999 \\
\hline \multirow[t]{6}{*}{10} & 10 & e & 1 & .0500 & .5126 & .8097 & .9600 & 9898 & 1.0000 & 1.0000 \\
\hline & & & 2 & .0500 & .5126 & .8097 & .9699 & .9998 & 1.0000 & 1.0000 \\
\hline & & 5 & 1 & .0500 & .8620 & .9783 & .9986 & 1.0000 & 1.0000 & 1.0000 \\
\hline & & & 2 & .0500 & .8620 & .9783 & .9986 & 1.0000 & 1.0000 & 1.0000 \\
\hline & & 10 & 1 & .0500 & .9748 & .9978 & .9898 & 1.0000 & 1.0000 & 1.0000 \\
\hline & & & 2 & .0500 & 9748 & 9978 & 9999 & 1.0000 & 1.0000 & 1.0000 \\
\hline
\end{tabular}


TABELA A. 6. Probabilidades do erro tipo II, para as hipóteses $H^{(1)} e H^{(2)}$, ao nivel nominal de significancia 0,05 segundo os vários valores de $r, s, k, p$, $\lambda=0 ; 5$ e 20 .

\begin{tabular}{|c|c|c|c|c|c|c|c|c|c|c|}
\hline & $\lambda$ & $=0$ & & & & & $p$ & & & \\
\hline$r$ & $s$ & $\mathrm{~K}$ & $\mathrm{H}$ & .0000 & .0500 & .1000 & .2000 & .5000 & .7000 & .9000 \\
\hline 2 & 3 & 3 & 1 & 9500 & - & - & - & .4913 & .3455 & .1838 \\
\hline & & & 2 & .9500 & - & - & - & .4024 & .2173 & 0654 \\
\hline & & 5 & 1 & .9500 & - & - & .6460 & 3885 & .2855 & .1386 \\
\hline & & & 2 & .9500 & - & - & 6050 & .2613 & .1287 & .0363 \\
\hline & & 10 & 1 & .9500 & - & .6592 & .5045 & .2792 & .1867 & .0964 \\
\hline & & & 12 & .9500 & - & .6175 & 4056 & .1388 & .0639 & .0172 \\
\hline $\bar{z}$ & 5 & 3 & 1 & .9500 & $\overline{-}$ & - & .6453 & .3882 & .2653 & .1385 \\
\hline & & & 12 & 9500 & - & - & .6189 & .1873 & .0549 & .0050 \\
\hline & & 5 & 1 & 9500 & - & .6945 & 5418 & .3054 & .2051 & 1061 \\
\hline & & & 2 & .9500 & - & .6877 & 4302 & .0832 & .0203 & 0016 \\
\hline & & 10 & 1 & .9500 & 6991 & .5593 & .4092 & .2181 & .1446 & 0741 \\
\hline & & & 2 & .9500 & .6833 & .4483 & 1990 & .0238 & .0051 & .0004 \\
\hline 5 & 3 & 2 & 1 & .9500 & $\overline{-}$ & .8111 & 6286 & .1934 & .0571 & .0052 \\
\hline & & & 2 & .9500 & - & .7803 & .6085 & .2642 & .1303 & .0368 \\
\hline & & 5 & 1 & .9500 & 7612 & .5586 & .2878 & .0413 & .0092 & .0007 \\
\hline & & & a & .9500 & .7365 & .5612 & .3506 & .1140 & .0517 & .0138 \\
\hline & & 10 & 1 & .9500 & .5453 & .2984 & .1087 & 0107 & .0022 & .0002 \\
\hline & & & 2 & .9500 & 5602 & .3663 & .1890 & .0571 & .0252 & .0067 \\
\hline 5 & 5 & 2 & 1 & .9500 & .8337 & .6894 & .4335 & .0843 & .0207 & .0017 \\
\hline & & & 2 & .9500 & .8337 & .6884 & .4335 & 0843 & .0207 & .0017 \\
\hline & & 5 & 1 & .9500 & .6194 & .3709 & 1481 & .0159 & .0033 & .0002 \\
\hline & & & 2 & .9500 & .6184 & .3709 & 1481 & 0159 & .0033 & .0002 \\
\hline & & 10 & 1 & .9500 & .3744 & .1641 & .0492 & .0041 & .0008 & .0001 \\
\hline & & & 2 & .9500 & 3744 & .1641 & 0492 & .0041 & .0008 & .0001 \\
\hline 10 & 10 & 2 & 11 & .9500 & .4874 & .1903 & .0301 & .0002 & .0000 & .0000 \\
\hline & & & 12 & .9500 & .4874 & .1903 & .0301 & .0002 & .0000 & .0000 \\
\hline & & 5 & 1 & .9500 & .1380 & .0217 & .0014 & 0000 & .0000 & .0000 \\
\hline & & & 2 & .9500 & 1380 & .0217 & .0014 & 0000 & .0000 & .0000 \\
\hline & & 10 & 1 & .9500 & .0252 & .0022 & .0001 & .0000 & .0000 & .0000 \\
\hline & & & 2 & .9500 & .0252 & .0022 & .0001 & .0000 & .0000 & .0000 \\
\hline
\end{tabular}


TABELA A.6. Continuação para $\lambda=5$.

\begin{tabular}{|c|c|c|c|c|c|c|c|c|c|c|}
\hline \multicolumn{4}{|c|}{$\lambda=5$} & \multicolumn{6}{|c|}{$\bar{\rho}$} & \multirow[b]{2}{*}{.8000} \\
\hline$r$ & Is & lk & $\mathrm{H}^{*}$ & .0000 & .0500 & .1000 & .2000 & .5000 & .7000 & \\
\hline \multirow[t]{6}{*}{$\bar{z}$} & 13 & 3 & 11 & - & $\overline{-}$ & - & $\overline{-}$ & .0649 & .0482 & .0390 \\
\hline & & & 2 & - & - & - & - & .0653 & .0341 & .0184 \\
\hline & & 5 & 1 & - & - & - & .0969 & .0516 & .0428 & .0379 \\
\hline & & & 2 & - & - & - & .1246 & .0395 & .0249 & .0178 \\
\hline & & 10 & 1 & - & - & .0988 & .0650 & .0435 & .0384 & .0372 \\
\hline & & & 2 & - & - & .1254 & .0626 & .0260 & .0200 & .0169 \\
\hline \multirow[t]{6}{*}{$\bar{z}$} & 5 & 3 & 1 & - & - & - & .0970 & .0516 & .0427 & .0379 \\
\hline & & & 2 & - & - & - & .1980 & .0327 & .0107 & .0036 \\
\hline & & 5 & 1 & - & - & .1115 & .0713 & .0451 & .0401 & .0373 \\
\hline & & & 2 & - & - & .2359 & .0974 & .0147 & .0061 & .0030 \\
\hline & & 10 & 1 & 3804 & .1116 & .0730 & .0532 & .0406 & .0382 & .0368 \\
\hline & & & 12 & 6197 & .2251 & .1012 & .0338 & .0067 & .0039 & .0027 \\
\hline \multirow[t]{6}{*}{5} & 3 & 12 & 1 & - & - & .3814 & .2056 & .0340 & .0110 & .0036 \\
\hline & & & 2 & - & - & .2382 & .1272 & .0300 & .0250 & .0178 \\
\hline & & 5 & 1 & 6346 & 2958 & .1505 & .0533 & .0090 & .0046 & .0028 \\
\hline & & & 2 & 5146 & .1892 & 1034 & .0523 & .0239 & .0192 & .0168 \\
\hline & & 10 & 1 & .5881 & .1408 & .0552 & .0185 & .0049 & .0033 & .0026 \\
\hline & & & 2 & .4914 & .1018 & .0547 & .0318 & .0197 & .0176 & .0165 \\
\hline \multirow[t]{6}{*}{5} & 5 & ट & 1 & .6466 & .3928 & .2392 & .0990 & .0148 & .0062 & .0030 \\
\hline & & & 2 & 6466 & .3928 & .2382 & .0990 & .0140 & .0062 & .0030 \\
\hline & & 5 & 1 & .6185 & .1813 & .0751 & .0247 & .0056 & .0036 & .0026 \\
\hline & & & 2 & .6185 & 1813 & .0751 & .0247 & .0056 & .0036 & .0026 \\
\hline & & 10 & 1 & 6000 & .0754 & .0273 & .0102 & .0038 & .0030 & .0025 \\
\hline & & & le & 6000 & .0754 & .0273 & .0102 & .0038 & .0030 & .0025 \\
\hline \multirow[t]{6}{*}{101} & 110 & 2 & 1 & .7106 & .1745 & .0450 & .0055 & .0001 & .0000 & .0000 \\
\hline & & & 2 & .7106 & .1745 & .0450 & .0055 & 0001 & 0000 & .0000 \\
\hline & & 5 & 1 & .7160 & . 0296 & .0039 & .0004 & .0000 & .0000 & .0000 \\
\hline & & & 2 & .7160 & .0296 & .0039 & .0004 & .0000 & .0000 & .0000 \\
\hline & & 10 & 1 & .7178 & .0045 & .0005 & .0001 & .0000 & 0000 & 0000 \\
\hline & & & 2 & .7178 & 0045 & 0005 & .0001 & 0000 & 0000 & 0000 \\
\hline
\end{tabular}


TABELA A. 6. Continuaçăo para $\lambda=20$.

\begin{tabular}{|c|c|c|c|c|c|c|c|c|c|c|}
\hline & $\lambda$ & $=$ & 80 & & & & $p$ & & & \\
\hline$r$ & Is & IK & $\mathrm{CH}^{*}$ & 0000 & .0500 & .1000 & .2000 & .5000 & .7000 & .9000 \\
\hline $\bar{z}$ & $\sqrt{3}$ & 13 & 1 & - & - & - & - & .0003 & .0002 & .0001 \\
\hline & & & 12 & - & - & - & - & .0004 & .0001 & .0001 \\
\hline & & 5 & 1 & - & - & - & .0008 & .0003 & .0002 & .0002 \\
\hline & & & 12 & - & - & - & .0011 & .0002 & .0001 & .0001 \\
\hline & & 10 & 1 & - & - & .0007 & .0004 & .0003 & .0003 & .0002 \\
\hline & & & 2 & - & - & .0011 & .0005 & .0002 & .0001 & .0001 \\
\hline$\overline{2}$ & 5 & 3 & 11 & - & - & - & .0006 & .0003 & .0003 & .0002 \\
\hline & & & le & - & - & - & .0036 & .0002 & .0001 & .0000 \\
\hline & & 5 & 1 & - & - & .0008 & .0005 & .0003 & .0003 & .0002 \\
\hline & & & 12 & - & - & .0046 & .0010 & .0001 & .0001 & .0000 \\
\hline & & 10 & 1 & .0080 & .0009 & .0005 & .0004 & .0003 & .0003 & .0003 \\
\hline & & & 12 & .0450 & .0040 & .0011 & .0003 & .0001 & .0000 & .0000 \\
\hline$\overline{5}$ & 3 & 2 & 1 & - & - & .0155 & .0039 & .0002 & .0001 & .0000 \\
\hline & & & 2 & - & - & .0036 & .0011 & .0002 & .0001 & .0001 \\
\hline & & 5 & 1 & .0510 & .0070 & .0020 & .0005 & .0001 & .0000 & .0000 \\
\hline & & & 12 & .0202 & .0022 & .0000 & .0004 & .0002 & .0001 & .0001 \\
\hline & & 10 & 1 & .0386 & .0018 & .0005 & .0002 & .0001 & .0000 & .0000 \\
\hline & & & 12 & .0157 & .0009 & .0004 & .0003 & .0002 & .0002 & .0002 \\
\hline 5 & 5 & 2 & 11 & .0603 & .0143 & .0048 & .0010 & .0001 & .0000 & .0000 \\
\hline & & & 2 & .0603 & .0143 & .0048 & .0010 & .0001 & .0000 & .0000 \\
\hline & & 5 & 1 & .0437 & .0027 & .0007 & .0002 & .0001 & .0000 & .0000 \\
\hline & & & 2 & .0437 & .0027 & .0007 & .0002 & .0001 & .0000 & .0000 \\
\hline & & 10 & 1 & .0377 & .0007 & .0002 & .0001 & .0001 & .0000 & .0000 \\
\hline & & & 12 & .0377 & .0007 & .0002 & .0001 & .0001 & .0000 & .0000 \\
\hline$\overline{10}$ & 10 & 2 & 1 & .1017 & .0041 & .0005 & .0001 & .0000 & .0000 & .0000 \\
\hline & & & 2 & 1017 & .0041 & .0005 & .0001 & .0000 & .0000 & .0000 \\
\hline & & 5 & 1 & .0985 & .0003 & .0000 & .0000 & .0000 & .0000 & .0000 \\
\hline & & & a & .0985 & .0003 & .0000 & .0000 & .0000 & .0000 & .0000 \\
\hline & & 10 & 1 & .0975 & .0000 & .0000 & .0000 & .0000 & .0000 & .0000 \\
\hline & & & 12 & .0975 & .0000 & .0000 & .0000 & .0000 & .0000 & .0000 \\
\hline
\end{tabular}


TABELA A.7. Frobabilidades do erro tipo I, para a hipotese $\mathrm{H}_{0}^{(1)}$, ao nivel nominal de significancia 0.05 , segundo os valores de $r, s, k$ e $p$.

\begin{tabular}{|c|c|c|c|c|c|c|c|c|c|}
\hline & & & & & & $\rho$ & & & \\
\hline$r$ & $s$ & $k$ & -.2000 & -1000 & -.0500 & .0000 & 1000 & 5000 & 9000 \\
\hline \multirow[t]{3}{*}{2} & 3 & 3 & - & .0002 & .0132 & .0500 & .1516 & .5087 & .8162 \\
\hline & & 5 & - & - & .0007 & .0500 & 2191 & 6115 & 8614 \\
\hline & & 10 & - & - & - & .0500 & .3408 & .7208 & 9036 \\
\hline \multirow[t]{3}{*}{ ᄅ } & 5 & 3 & - & - & .0003 & .0500 & .2200 & .6118 & 8615 \\
\hline & & 5 & - & - & - & .0500 & .3055 & .6946 & 8939 \\
\hline & & 10 & - & - & - & .0500 & .4407 & .7819 & 9959 \\
\hline \multirow[t]{3}{*}{5} & 3 & 2 & - & - & .0143 & .0500 & .1889 & .8066 & 9948 \\
\hline & & 5 & - & - & .0000 & .0500 & 4414 & 9587 & 9993 \\
\hline & & 10 & - & - & - & .0500 & .7016 & 9893 & 9998 \\
\hline \multirow[t]{3}{*}{5} & 5 & 2 & - & - & - & .0500 & .3106 & 9157 & .9983 \\
\hline & & 5 & - & - & - & .0500 & 6291 & 9841 & 9998 \\
\hline & & 10 & - & - & - & .0500 & .8359 & 9959 & . 9999 \\
\hline \multirow[t]{3}{*}{10} & 10 & 2 & - & - & - & .0500 & .8097 & .9998 & 1.0000 \\
\hline & & 5 & - & - & - & .0500 & .9783 & 1.0000 & 1.0000 \\
\hline & & 10 & - & - & - & .0500 & .9978 & 1.0000 & 1.0000 \\
\hline
\end{tabular}

TABELA A.8. Probabilidades do erro tipo II, para a hipotese $H_{0}^{(1)}$, 20 nivel nominal de significancia 0.05 , segundo os valores de $r, s, k, \rho, \lambda=0 ; 5 e 20$.

\begin{tabular}{|c|c|c|c|c|c|c|c|c|c|}
\hline \multicolumn{3}{|c|}{$\lambda=0$} & \multicolumn{7}{|c|}{$p$} \\
\hline$r$ & $s$ & K & -.2000 & -.1000 & -.0500 & .0000 & .1000 & 5000 & .9000 \\
\hline \multirow[t]{3}{*}{2} & 3 & 3 & - & .9998 & .9868 & .9500 & .8484 & .4943 & .1838 \\
\hline & & 5 & - & - & .9993 & .9500 & .7809 & .3885 & .1386 \\
\hline & & 10 & - & - & - & .9500 & .6592 & 2792 & .0964 \\
\hline \multirow[t]{3}{*}{2} & 5 & 3 & - & $=$ & .9992 & .9500 & .7800 & 3882 & .1385 \\
\hline & & 5 & - & - & - & .9500 & .6945 & 3054 & 1061 \\
\hline & & 10 & - & - & - & .9500 & .5593 & .2181 & .0741 \\
\hline \multirow[t]{3}{*}{5} & 3 & $\bar{z}$ & - & - & .9857 & .8500 & .8111 & 1934 & .0052 \\
\hline & & 5 & - & - & 1.0000 & .9500 & .5586 & .0413 & .0007 \\
\hline & & 10 & - & - & - & .9500 & .2984 & .0107 & .0002 \\
\hline \multirow[t]{3}{*}{5} & 5 & 2 & - & - & - & .9500 & .6894 & .0843 & .0017 \\
\hline & & 5 & - & - & - & .9500 & 3709 & .0159 & .0002 \\
\hline & & 10 & - & - & - & 9500 & 1641 & .0041 & .0001 \\
\hline \multirow[t]{3}{*}{10} & 10 & 2 & - & - & - & .9500 & .1903 & .0002 & .0000 \\
\hline & & 5 & - & - & - & .9500 & .0217 & .0000 & .0000 \\
\hline & & 10 & - & - & - & .9500 & .0022 & .0000 & .0000 \\
\hline
\end{tabular}


TABELA A.8. Continuação para $\lambda=5$.

\begin{tabular}{|c|c|c|c|c|c|c|c|c|c|}
\hline \multicolumn{3}{|c|}{$x=5$} & \multicolumn{7}{|c|}{$\rho$} \\
\hline$r$ & $s$ & K & -.2000 & -1000 & -.0500 & .0000 & .1000 & .5000 & .9000 \\
\hline \multirow[t]{3}{*}{2} & 3 & 3 & - & .9775 & .6852 & 4461 & .2284 & .0640 & .0390 \\
\hline & & 5 & - & - & .9193 & .4169 & .1578 & .0516 & .0379 \\
\hline & & 10 & - & - & - & .3968 & .0988 & .0435 & .0372 \\
\hline \multirow[t]{3}{*}{2} & 5 & 3 & - & - & .9171 & .4166 & .1580 & $.051 \%$ & .0379 \\
\hline & & 5 & - & - & - & .4022 & .1115 & .0451 & .0373 \\
\hline & & 10 & - & - & - & 3904 & .0739 & .0405 & .0369 \\
\hline \multirow[t]{3}{*}{5} & 3 & 2 & - & - & .8398 & .6731 & .3814 & .0340 & .0036 \\
\hline & & 5 & - & - & .9982 & .6346 & .1505 & .0090 & .0028 \\
\hline & & 10 & - & - & - & .5981 & .0552 & .0049 & .0026 \\
\hline \multirow[t]{3}{*}{5} & 5 & 2 & - & - & - & .6466 & .2392 & .0149 & .0030 \\
\hline & & 5 & - & - & - & .6185 & .0751 & .0050 & .0026 \\
\hline & & 10 & - & - & - & .6000 & .0273 & .0038 & .0025 \\
\hline \multirow[t]{3}{*}{10} & 10 & 2 & - & - & $\overline{-}$ & .7106 & .0450 & .0001 & .0000 \\
\hline & & 5 & - & - & - & .7160 & .0039 & .0000 & .0000 \\
\hline & & 10 & - & - & - & .7178 & .0005 & .0000 & .0000 \\
\hline
\end{tabular}

TABELA A.8. Continuação ara $\lambda=20$.

\begin{tabular}{|c|c|c|c|c|c|c|c|c|c|}
\hline \multicolumn{3}{|c|}{$\lambda=5$} & \multicolumn{7}{|c|}{ 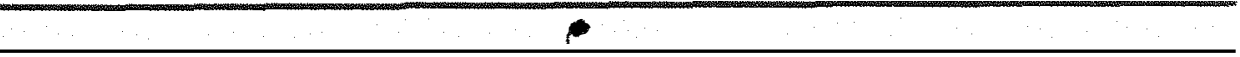 } \\
\hline$r$ & $s$ & $\kappa$ & -.2000 & -.1000 & -.0500 & .0000 & .1000 & .5000 & .9000 \\
\hline \multirow[t]{3}{*}{2} & 3 & 3 & - & .6298 & .0690 & .0150 & .0026 & .0003 & .0001 \\
\hline & & 5 & - & - & .2780 & .0106 & .0013 & .0003 & .0002 \\
\hline & & 10 & - & - & - & .0086 & .0007 & .0003 & .0002 \\
\hline \multirow[t]{3}{*}{2} & 5 & 3 & - & - & 2789 & .0107 & .0013 & .0003 & .0002 \\
\hline & & 5 & - & - & - & .0090 & .0008 & .0003 & .0002 \\
\hline & & 10 & - & - & - & .0080 & .0005 & .0003 & .0003 \\
\hline \multirow[t]{3}{*}{5} & 3 & 2 & - & - & .2150 & .0837 & .0155 & .0002 & 0000 \\
\hline & & 5 & - & - & 8495 & .0510 & $.00 \geq 0$ & .0001 & .0000 \\
\hline & & 10 & - & - & - & .0386 & .0005 & .0001 & .0000 \\
\hline \multirow[t]{3}{*}{5} & 5 & 2 & - & - & - & .0503 & .0048 & .0001 & .0000 \\
\hline & & 5 & - & - & - & .0437 & .0007 & .0001 & .0000 \\
\hline & & 10 & - & - & - & .0377 & .0002 & .0001 & .0000 \\
\hline \multirow[t]{3}{*}{10} & 10 & 2 & - & - & - & .1017 & .0005 & .0000 & .0000 \\
\hline & & 5 & - & - & - & .0985 & .0000 & .0000 & 0000 \\
\hline & & 10 & - & - & - & .0975 & .0000 & .0000 & .0000 \\
\hline
\end{tabular}


TABELA A. O. Frobabilidades do erro tipo I, para as hipoteses $\mathrm{H}^{\prime \prime}{ }^{\prime} \mathrm{Ho}^{\langle 2}$ e $\mathrm{Ho}^{\left\langle{ }^{\prime}\right\rangle}$ ao nivel nominal de significancia 0,05 segundo os vários valores de $r, s, k e p$.

\begin{tabular}{|c|c|c|c|c|c|c|c|c|c|c|}
\hline \multirow[b]{2}{*}{$r$} & \multirow[b]{2}{*}{$s$} & \multirow[b]{2}{*}{ K } & \multirow[b]{2}{*}{$\mathrm{H}^{*}$} & \multicolumn{7}{|c|}{$\rho$} \\
\hline & & & & -.8000 & -.4000 & -.1000 & .0000 & .1000 & .4000 & .8000 \\
\hline \multirow[t]{9}{*}{2} & \multirow[t]{9}{*}{3} & \multirow[t]{3}{*}{3} & 1 & - & .0001 & .0252 & .0500 & .0835 & .2322 & .5568 \\
\hline & & & 2 & - & .0000 & .0218 & .0500 & .0928 & 3091 & .7468 \\
\hline & & & 3 & - & .0000 & .0218 & .0500 & .0928 & 3091 & .7468 \\
\hline & & \multirow[t]{3}{*}{5} & 1 & - & - & .0102 & .0500 & .1118 & 3324 & .6572 \\
\hline & & & 2 & - & - & .0087 & .0500 & .1350 & 4687 & 8519 \\
\hline & & & 3 & - & - & .0067 & .0500 & .1350 & 4687 & 8519 \\
\hline & & \multirow[t]{3}{*}{10} & 1 & - & - & .0000 & .0500 & .1744 & .4732 & .7559 \\
\hline & & & 2 & - & - & .0000 & .0500 & .2340 & 6651 & .8262 \\
\hline & & & 3 & - & - & 0000 & .0500 & .2340 & .6651 & 9262 \\
\hline \multirow[t]{9}{*}{$\overline{\mathrm{C}}$} & \multirow[t]{9}{*}{5} & \multirow[t]{3}{*}{3} & 1 & - & .0000 & .0238 & .0500 & .0860 & .2426 & 5684 \\
\hline & & & 2 & - & .0000 & .0161 & .0500 & 1117 & 4525 & 8237 \\
\hline & & & 3 & - & .0000 & .0161 & .0500 & .1117 & 4525 & 9237 \\
\hline & & \multirow[t]{3}{*}{5} & 1 & - & - & .0093 & .0500 & .1132 & 3377 & 6617 \\
\hline & & & 2 & - & - & .0030 & .0500 & .1741 & 6632 & 9729 \\
\hline & & & 3 & - & - & .0030 & .0500 & 1741 & .6632 & 9729 \\
\hline & & \multirow[t]{3}{*}{10} & 1 & - & - & .0000 & .0500 & .1764 & .4764 & 7578 \\
\hline & & & 2 & - & - & .0000 & .0500 & .3336 & .8642 & 9933 \\
\hline & & & 3 & - & - & .0000 & .0500 & .3336 & .8642 & 9933 \\
\hline \multirow[t]{9}{*}{5} & \multirow[t]{9}{*}{3} & \multirow[t]{3}{*}{2} & 1 & 0000 & .0020 & .0265 & .0500 & .0864 & .3105 & .8467 \\
\hline & & & 2 & 0000 & .0033 & .0295 & .0500 & .0796 & 2390 & .6716 \\
\hline & & & 3 & .0000 & .0013 & .0242 & .0500 & .0945 & .3976 & 9574 \\
\hline & & \multirow[t]{3}{*}{5} & 1 & - & - & .0025 & .0500 & .1793 & 6756 & 9747 \\
\hline & & & 2 & - & - & .0051 & .0500 & .1409 & 4876 & 8610 \\
\hline & & & 3 & - & - & .0010 & .0500 & .2372 & 8624 & 9991 \\
\hline & & \multirow[t]{3}{*}{10} & 1 & - & - & .0000 & .0500 & 3419 & 8689 & 9835 \\
\hline & & & 2 & - & - & .0000 & .0500 & .2428 & 6752 & 9290 \\
\hline & & & 3 & - & - & .0000 & .0500 & .4953 & 9786 & 9990 \\
\hline 5 & 5 & 2 & 1 & .0000 & .0010 & .0244 & .0500 & .0914 & . 3424 & 8707 \\
\hline & & & 2 & 0000 & .0010 & .0244 & .0500 & .0914 & 3424 & 8707 \\
\hline & & & 3 & 0000 & 0002 & .0173 & .0500 & .1112 & 5816 & 9981 \\
\hline & & 5 & 1 & - & - & .0022 & .0500 & .1868 & 6883 & 9753 \\
\hline & & & 2 & - & - & .0022 & .0500 & .1868 & 6883 & .9763 \\
\hline & & & 3 & - & - & .0002 & .0500 & .3431 & .9788 & 1.0000 \\
\hline & & 10 & 1 & - & - & .0000 & .0500 & .3466 & 8717 & 9938 \\
\hline & & & 2 & - & - & .0000 & .0500 & 3466 & 8717 & .9938 \\
\hline & & & 3 & - & - & .0000 & .0500 & 5950 & 9995 & 1.0000 \\
\hline$\overline{10}$ & 10 & 2 & 1 & 0000 & 0001 & .0217 & .0500 & .1449 & 6120 & 9825 \\
\hline & & & 2 & 0000 & 0001 & .0217 & .0500 & .1449 & 6120 & 9926 \\
\hline & & & 3 & 0000 & 0000 & .0065 & .0500 & 2805 & 9925 & 1.0000 \\
\hline & & 5 & 1 & - & - & .0004 & .0500 & 2870 & 0167 & .0088 \\
\hline & & & 2 & - & - & .0004 & .0500 & 2879 & .9167 & 9998 \\
\hline & & & 3 & - & - & .0000 & .0500 & 9086 & 1.0000 & 1.0000 \\
\hline & & 10 & 1 & - & - & .0000 & .0500 & 5332 & .9876 & 1.0000 \\
\hline & & & 2 & - & - & .0000 & .0500 & 5332 & 9876 & 1.0000 \\
\hline & & & 3 & - & - & .0000 & .0500 & 9989 & 1.0000 & 1.0000 \\
\hline
\end{tabular}


IABELA A.10. Probabilidades do erro tipo II, para as hipóteses $H^{\prime 2 !} H^{(2)}$ e $H^{(3)}$ ao nivel nominal de significancia 0,05 segundo os valores de $r, s, k, \rho, \lambda=0 ; 5$ e 20

\begin{tabular}{|c|c|c|c|c|c|c|c|c|c|c|}
\hline \multicolumn{4}{|c|}{$\lambda=0$} & \multicolumn{7}{|c|}{$\rho$} \\
\hline & s & $k$ & $\mathrm{H}^{*}$ & $1-.8000$ & -.4000 & -.1000 & .0000 & 1000 & 4000 & 8000 \\
\hline & 3 & 10 & $\begin{array}{l}1 \\
2 \\
3 \\
1 \\
2 \\
3 \\
1 \\
2 \\
3\end{array}$ & $\begin{array}{l}- \\
- \\
- \\
- \\
- \\
- \\
- \\
-\end{array}$ & $\begin{array}{c}.99595 \\
1.0000 \\
1.0000 \\
- \\
- \\
- \\
- \\
-\end{array}$ & $\begin{array}{r}.9748 \\
.9782 \\
.9782 \\
.9898 \\
.9898 \\
.9933 \\
1.0000 \\
1.0000 \\
1.0000\end{array}$ & $\begin{array}{l}.9500 \\
.9500 \\
.9500 \\
.9500 \\
.8500 \\
.9500 \\
.8500 \\
.9500 \\
.8500\end{array}$ & $\begin{array}{l}.9165 \\
.9072 \\
.9072 \\
.8882 \\
.8882 \\
.8650 \\
.8256 \\
7660 \\
.7660\end{array}$ & $\begin{array}{l}.7678 \\
6909 \\
.6909 \\
6676 \\
.6676 \\
.5313 \\
.5268 \\
3349 \\
.3349\end{array}$ & $\begin{array}{l}.4432 \\
2532 \\
.2532 \\
3428 \\
.3428 \\
1481 \\
.2441 \\
.0738 \\
.0738\end{array}$ \\
\hline 2 & 5 & 10 & $\begin{array}{l}1 \\
2 \\
3 \\
1 \\
2 \\
3 \\
1 \\
2 \\
3\end{array}$ & $\begin{array}{l}- \\
- \\
- \\
- \\
- \\
- \\
-\end{array}$ & $\begin{array}{c}1.0000 \\
1.0000 \\
1.0000 \\
- \\
- \\
- \\
- \\
-\end{array}$ & $\begin{array}{r}.9762 \\
.9839 \\
.9839 \\
.9907 \\
.9970 \\
.9970 \\
1.0000 \\
1.0000 \\
1.0000\end{array}$ & $\begin{array}{l}.9500 \\
.9500 \\
.9500 \\
.9500 \\
.9500 \\
.9500 \\
.9500 \\
.8500 \\
.9500\end{array}$ & $\begin{array}{l}.8140 \\
.8883 \\
.8833 \\
.8868 \\
.8259 \\
.8259 \\
.8236 \\
.6664 \\
.6664\end{array}$ & $\begin{array}{r}.7574 \\
.5475 \\
.5475 \\
6623 \\
.3368 \\
.3368 \\
5236 \\
1358 \\
1358\end{array}$ & $\begin{array}{l}.4306 \\
.0763 \\
.0763 \\
.3338 \\
.0271 \\
.0271 \\
.2421 \\
.0067 \\
.0067\end{array}$ \\
\hline & & 10 & $\begin{array}{l}1 \\
2 \\
3 \\
1 \\
2 \\
3 \\
1 \\
2 \\
3\end{array}$ & $\begin{array}{c}1.0000 \\
1.0000 \\
1.0000 \\
- \\
- \\
- \\
- \\
-\end{array}$ & $\begin{array}{c}.9980 \\
.9867 \\
.9987 \\
- \\
- \\
- \\
- \\
- \\
-\end{array}$ & $\begin{array}{r}.9735 \\
.9705 \\
.9758 \\
.9875 \\
.9949 \\
.9990 \\
1.0000 \\
1.0000 \\
1.0000\end{array}$ & $\begin{array}{l}.9500 \\
.9500 \\
.9500 \\
.9500 \\
9500 \\
.9500 \\
.9500 \\
.9500 \\
.9500\end{array}$ & $\begin{array}{l}.9136 \\
9204 \\
.9055 \\
.8207 \\
.8591 \\
.7628 \\
.6581 \\
.7572 \\
.5047\end{array}$ & $\begin{array}{l}.6885 \\
.7610 \\
.6024 \\
.3244 \\
5124 \\
1376 \\
1311 \\
3248 \\
.0214\end{array}$ & $\begin{array}{l}.1533 \\
.3284 \\
.0426 \\
.0253 \\
1390 \\
.0009 \\
.0064 \\
.0710 \\
.0214\end{array}$ \\
\hline & & 10 & $\begin{array}{l}1 \\
2 \\
3 \\
1 \\
2 \\
3 \\
1 \\
2 \\
3\end{array}$ & $\begin{array}{c}1.0000 \\
1.0000 \\
1.0000 \\
- \\
- \\
- \\
- \\
-\end{array}$ & $\begin{array}{c}.9990 \\
9990 \\
9998 \\
- \\
- \\
- \\
- \\
- \\
-\end{array}$ & $\begin{array}{r}.9750 \\
.9756 \\
.9827 \\
.9878 \\
.9978 \\
.9998 \\
1.0000 \\
1.0000 \\
1.0000\end{array}$ & $\begin{array}{l}.9500 \\
.9500 \\
.9500 \\
.9500 \\
.9500 \\
.9500 \\
.9500 \\
.9500 \\
.9500\end{array}$ & $\begin{array}{l}.9086 \\
.9086 \\
.8888 \\
.8132 \\
.8132 \\
6569 \\
.6534 \\
6534 \\
3050\end{array}$ & $\begin{array}{l}.6576 \\
6576 \\
.4184 \\
3117 \\
.3117 \\
.0212 \\
1283 \\
1283 \\
3335\end{array}$ & $\begin{array}{l}.1293 \\
.1293 \\
.0019 \\
.0237 \\
.0237 \\
.0000 \\
.0062 \\
.0062 \\
.0000\end{array}$ \\
\hline 10 & 10 & 10 & $\begin{array}{l}1 \\
2 \\
3 \\
1 \\
2 \\
3 \\
1 \\
2 \\
3\end{array}$ & $\begin{array}{c}1.0000 \\
1.0000 \\
1.0000 \\
- \\
- \\
- \\
- \\
- \\
-\end{array}$ & $\begin{array}{c}.8999 \\
.8999 \\
1.0000 \\
- \\
- \\
- \\
- \\
- \\
-\end{array}$ & $\begin{array}{r}.9783 \\
.9783 \\
.9835 \\
.9996 \\
.9996 \\
1.0000 \\
1.0000 \\
1.0000 \\
1.0000\end{array}$ & $\begin{array}{l}.9500 \\
.9500 \\
.8500 \\
.9500 \\
.9500 \\
.9500 \\
.9500 \\
.9500 \\
.9500\end{array}$ & $\begin{array}{l}.8551 \\
.8551 \\
.7195 \\
.7121 \\
.7121 \\
.0914 \\
.4668 \\
.4668 \\
.0011\end{array}$ & $\begin{array}{l}.3880 \\
.3880 \\
.0075 \\
.0833 \\
.0833 \\
.0000 \\
0124 \\
.0124 \\
.0000\end{array}$ & $\begin{array}{l}.0074 \\
.0074 \\
.0000 \\
.0002 \\
.0002 \\
.0000 \\
.0000 \\
.0000 \\
.0000\end{array}$ \\
\hline
\end{tabular}


TABELAS A.10. Continuação para $\lambda=5$

\begin{tabular}{|c|c|c|c|c|c|c|c|c|c|c|}
\hline \multicolumn{4}{|c|}{$x=5$} & \multicolumn{7}{|c|}{$\rho$} \\
\hline$r$ & $s$ & I & $H^{*}$ & -.8000 & -.4000 & -.1000 & .0000 & .1000 & .4000 & .8000 \\
\hline \multirow[t]{9}{*}{$\bar{e}$} & 3 & 13 & 1 & - & 9891 & .5901 & .4570 & .3511 & .1591 & .0584 \\
\hline & & & 2 & - & 9978 & .7373 & .5937 & .4603 & .1833 & .0390 \\
\hline & & & 3 & - & .9978 & .7373 & .5937 & .4603 & .1833 & .0390 \\
\hline & & 5 & 1 & - & - & .6859 & .4175 & .2695 & .1042 & .0478 \\
\hline & & & e & - & - & . 8297 & .5432 & .3405 & .0976 & .0267 \\
\hline & & & 3 & - & - & .8297 & .5432 & .3405 & .0976 & .0267 \\
\hline & & 10 & 1 & - & - & 9999 & 3967 & 1859 & .0686 & .0417 \\
\hline & & & 2 & - & - & 1.0000 & .5134 & .2132 & .0498 & .0207 \\
\hline & & & 3 & - & - & 1.0000 & .5134 & .2132 & .0498 & .0207 \\
\hline \multirow[t]{9}{*}{$\bar{e}$} & 15 & 3 & 1 & - & 9932 & .5630 & .4267 & .3230 & .1459 & .0562 \\
\hline & & & 12 & - & 9999 & .8358 & 6817 & .5172 & .15 & .0137 \\
\hline & & & 3 & - & 9999 & .8358 & 6817 & .5172 & .1584 & .0137 \\
\hline & & 5 & 1 & - & - & .6775 & .4040 & .2585 & .1006 & .0473 \\
\hline & & & 2 & - & - & - 9269 & .6461 & .3824 & .0674 & .0070 \\
\hline & & & 3 & - & - & .9269 & .6461 & .3824 & .0674 & .0070 \\
\hline & & 10 & 1 & - & - & .0909 & .3884 & .1818 & .0677 & .0416 \\
\hline & & & 12 & - & - & 1.0000 & .6167 & . 21 32 & .0227 & .0042 \\
\hline & & & 3 & - & - & 1.0000 & .6167 & .2132 & .0227 & .0042 \\
\hline \multirow[t]{9}{*}{5} & 3 & 2 & 1 & 1.0000 & 9688 & .7974 & .7025 & .5948 & .2568 & .0267 \\
\hline & & & 12 & .9996 & 9088 & .6705 & .5742 & .4784 & .2318 & .0504 \\
\hline & & & 3 & 1.0000 & 9893 & .8858 & .8053 & .6980 & .2918 & .0082 \\
\hline & & 5 & 1 & - & - & .9270 & .6331 & .3762 & .0630 & .0069 \\
\hline & & & 12 & - & - & .8283 & .5140 & . 3121 & .0884 & .0250 \\
\hline & & & 3 & - & - & 9791 & .7393 & $\triangle O \geq 1$ & .0201 & .0003 \\
\hline & & 10 & 1 & - & - & 1.0000 & .6080 & .2052 & .0218 & .0042 \\
\hline & & & 2 & - & - & 1.0000 & 4948 & .2011 & .0479 & .0206 \\
\hline & & & 3 & - & - & 1.0000 & .7020 & .1761 & .0039 & .0001 \\
\hline \multirow[t]{9}{*}{5} & 15 & 2 & 1 & 1.0000 & 9705 & .7755 & .6576 & .5496 & .2244 & .0220 \\
\hline & & & 12 & 1.0000 & 9705 & .7755 & .6676 & .5496 & .2244 & .0224 \\
\hline & & & 13 & 1.0000 & 9985 & .9365 & .8581 & .7320 & .2099 & .0004 \\
\hline & & 5 & 1 & - & - & .9250 & .6175 & .3403 & .0588 & .0067 \\
\hline & & & 2 & - & - & .9250 & .6175 & .3483 & .0588 & .0067 \\
\hline & & & 13 & - & - & .9970 & .8113 & .3747 & .0045 & .0000 \\
\hline & & 10 & 1 & - & - & 1.0000 & .5998 & .2002 & .0214 & .0042 \\
\hline & & & 2 & - & - & 1.0000 & .5998 & .2002 & .0214 & .0042 \\
\hline & & & 13 & - & - & 1.0000 & .7874 & .1129 & .0001 & .0000 \\
\hline \multirow[t]{9}{*}{$\overline{10}$} & 110 & 2 & 1 & 1.0000 & .9966 & .8425 & .7038 & .5371 & .1241 & .0014 \\
\hline & & & 2 & 1.0000 & 9966 & .8425 & .7038 & .5371 & .1241 & .0014 \\
\hline & & & 3 & 1.0000 & 1.0000 & .9868 & .8942 & .8142 & .0034 & .0000 \\
\hline & & 5 & 1 & - & - & .9855 & .7153 & .3424 & .0163 & .0001 \\
\hline & & & 2 & - & - & .9855 & .7153 & .3424 & .0163 & .0001 \\
\hline & & & 3 & - & - & 1.0000 & 8042 & .0484 & .0000 & .0000 \\
\hline & & 10 & 1 & - & - & 1.0000 & .7176 & .1584 & .0023 & .0000 \\
\hline & & & 2 & - & - & 1.0000 & .7176 & .1584 & .0023 & .0000 \\
\hline & & & 3 & - & - & 1.0000 & .8185 & .0004 & .0000 & .0000 \\
\hline
\end{tabular}


TABELA A.10. Continuação pora $\lambda=20$

\begin{tabular}{|c|c|c|c|c|c|c|c|c|c|c|}
\hline & $\lambda$ & $=$ & 20 & & & & $\rho$ & & & \\
\hline$r$ & Is & $K$ & $\mathrm{H}^{*}$ & $1-.8000$ & -.4000 & -.1000 & .0000 & .1000 & .4000 & 8000 \\
\hline $\bar{z}$ & 13 & 3 & 1 & - & .7760 & .0411 & .0171 & .0079 & 0012 & .0002 \\
\hline & & & 12 & - & .9338 & 1225 & .0552 & .0226 & 0022 & .0002 \\
\hline & & & 3 & - & 9338 & .1225 & .0522 & .0226 & 0022 & .0002 \\
\hline & & 5 & 1 & - & - & .0564 & .0108 & .0037 & 0007 & .0002 \\
\hline & & & 2 & - & - & 1609 & .0290 & .0079 & 0007 & .0001 \\
\hline & & & 3 & - & - & 1609 & .0290 & .0079 & 0007 & .0001 \\
\hline & & 10 & 1 & - & - & .9644 & .0086 & .0018 & 0005 & .0003 \\
\hline & & & 12 & - & - & .9983 & .0206 & .0027 & 03 & .0002 \\
\hline & & & 3 & - & - & .9983 & .0200 & .0027 & 0003 & .0002 \\
\hline$\overline{2}$ & 5 & 3 & 1 & - & 7690 & .0283 & .0119 & .0057 & 0011 & .0003 \\
\hline & & & 12 & - & 9928 & .2213 & .08 & .03 & or & 01 \\
\hline & & & 3 & - & 9928 & .2213 & $.0 S$ & .0374 & 0 & .0 \\
\hline & & 5 & 1 & - & - & .0472 & .00 & .00 & .0 & .0003 \\
\hline & & & 2 & - & - & .3365 &.$O$ & .01 & .0 & 01 \\
\hline & & & 3 & - & - & .3365 &.$O B$ & .01 & .0 & 01 \\
\hline & & 10 & 1 & - & - & 9692 &.$O C$ & $O C$ & .0 & .0 \\
\hline & & & 2 & - & - & 1.0000 & .0 &.$O C$ & .0 & .0 \\
\hline & & & 3 & - & - & 1.0000 & .0446 & .00 &.$O C$ & .0000 \\
\hline 5 & 3 & 2 & 1 & 9970 & 6255 & .2003 & .1189 & .0668 & 0077 & .0002 \\
\hline & & & 2 & 9763 & 3381 & .0737 & .0416 & .0230 & $O C$ & 0003 \\
\hline & & & 3 & 9997 & 8597 & .4322 & .2886 & .1740 & .01 & .0001 \\
\hline & & 5 & 1 & - & - & .3108 & .0517 & .01 &.$O$ & .0001 \\
\hline & & & 2 & - & - & .1220 & .0204 &.$O C$ & .0 & .0 \\
\hline & & & 3 & - & - & .6193 & .1294 &. $\mathrm{O}^{\prime}$ &.$O S$ & $.0 C$ \\
\hline & & 10 & 1 & - & - & 1.0000 & .0404 & .00 & .0 & .0 \\
\hline & & & 2 & - & - & .9993 & .0171 &.$O C$ & .0 & \\
\hline & & & 3 & - & - & 1.0000 & $.0 S$ & 39 &.$O$ & .0000 \\
\hline 5 & 15 & 2 & 1 & 9990 & 5722 & .1428 & .0787 & .0412 & .0046 & .0001 \\
\hline & & & 2 & 9990 & 5722 & 1428 & .0787 & .04 &.$O S$ & .0001 \\
\hline & & & 3 & 1.0000 & 9694 & 6213 & .4296 & .25 & 01 & .0000 \\
\hline & & 5 & 1 & - & - & .2843 & .0442 & .0097 & .0005 & .0001 \\
\hline & & & 12 & - & - & .2843 & .0442 & .0097 & $O C$ & .0001 \\
\hline & & & 3 & - & - & .8829 & .2479 & .0321 & 0000 & .0000 \\
\hline & & 10 & 1 & - & - & 1. 0000 & .0378 & .00 & $O C$ & .0001 \\
\hline & & & 2 & - & - & 1.0000 & 0 & $O$ & .0 & .0 \\
\hline & & & 3 & - & - & 1.0000 & 20 & or & .0 & .0000 \\
\hline 10 & 10 & 2 & 1 & 1.0000 & 8268 & .2156 & .1057 & .0468 & .00 & .0000 \\
\hline & & & 2 & 1.0000 & 8268 & .2156 & .1057 & .04 & .0 & 0000 \\
\hline & & & 3 & 1,0000 & .0000 & .9265 & .6878 & $.3 a$ &.$O$ & 0000 \\
\hline & & 5 & 1 & - & - & 6119 & .0989 & .0143 & .00 & 0000 \\
\hline & & & 2 & - & - & 6119 & .08 & .01 & & \\
\hline & & & 3 & - & - & .9999 & 4632 & .00 & .00 & 0000 \\
\hline & & 10 & 1 & - & - & 1.0000 & .0976 & .00 & .00 & 0000 \\
\hline & & & 12 & - & - & 1.0000 & $.0 B$ & .00 & .0 & 0000 \\
\hline & & & 3 & 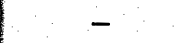 & 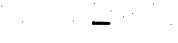 & 1.0000 & .4836 & 0000 & 0000 & 0000 \\
\hline
\end{tabular}


TAEELA A.11. Probabilidades do erro tipo I, para a hipotese $H_{0}^{(1)}$, ao nivel nominal de significancia 0.05 , segundo os valores de $r, s, k e p$.

\begin{tabular}{|c|c|c|c|c|c|c|c|c|c|}
\hline & & & & & & 0 & & & \\
\hline & 5 & K & -.2000 & -.1000 & -.0500 & .0000 & .1000 & 5000 & .9000 \\
\hline \multirow{3}{*}{$\frac{r}{2}$} & 3 & 3 & - & .0072 & .0242 & .0500 & .1172 & .4261 & .7738 \\
\hline & & 5 & - & .0054 & .0223 & .0500 & .1230 & .4429 & .7831 \\
\hline & & 10 & - & .0045 & .0216 & .0500 & .1272 & .4530 & .7885 \\
\hline \multirow[t]{3}{*}{2} & 5 & 3 & - & .0000 & .0082 & .0500 & .1660 & .5365 & .8202 \\
\hline & & 5 & - & .0000 & .0080 & .0500 & .1722 & .5460 & .8334 \\
\hline & & 10 & - & .0000 & .0075 & .0500 & .1764 & .5520 & .8360 \\
\hline \multirow[t]{3}{*}{5} & 3 & 2 & - & - & .0003 & .0500 & .3723 & .9393 & 8939 \\
\hline & & 5 & - & - & .0000 & .0500 & .4423 & 9586 & 9903 \\
\hline & & 10 & - & - & .0000 & .0500 & 4660 & 9627 & 9994 \\
\hline \multirow[t]{3}{*}{5} & 5 & 2 & - & - & - & .0500 & .5796 & 9793 & 9997 \\
\hline & & 5 & - & - & - & .05 & .6292 & 8841 & 9998 \\
\hline & & 10 & - & - & - & .0500 & .6434 & 8852 & 9998 \\
\hline \multirow[t]{3}{*}{10} & 10 & 2 & - & - & - & .0500 & .9972 & 1.0000 & 1.0000 \\
\hline & & 5 & - & - & - & .0500 & .9978 & 1.0000 & 1.0000 \\
\hline & & 10 & - & - & - & .0500 & . 9978 & 1.0000 & 1.0000 \\
\hline
\end{tabular}

TABELA A.12.Probabilidades do erro tipo II, para a hipótese $H_{0}^{(1)}$, ao nivel nominal de significância 0.05 , segundo os valores de $r, s, k, \rho, \lambda=0 ; 5$ e 20 .

\begin{tabular}{|c|c|c|c|c|c|c|c|c|c|}
\hline \multicolumn{3}{|c|}{$\lambda=0$} & \multicolumn{7}{|c|}{$\rho$} \\
\hline$r$ & $s$ & $\mathrm{~K}$ & -.2000 & -1000 & -.0500 & .0000 & .1000 & .5000 & .8000 \\
\hline \multirow[t]{3}{*}{2} & 3 & 3 & - & .9828 & .9758 & .9500 & .8828 & .5739 & .2262 \\
\hline & & 5 & - & 8946 & .9777 & .0500 & .8770 & .5571 & 2168 \\
\hline & & 10 & - & 9955 & .9784 & .9500 & .8728 & .5470 & 2115 \\
\hline \multirow[t]{3}{*}{2} & 5 & 3 & - & 1.0000 & .9908 & .9500 & .8334 & .4635 & .1708 \\
\hline & & 5 & - & 1.0000 & .9920 & .9500 & .8278 & .4540 & .1686 \\
\hline & & 10 & - & 1.0000 & .9925 & .9900 & .8236 & .4480 & .1640 \\
\hline \multirow[t]{3}{*}{5} & 3 & 2 & $=$ & $=$ & .9997 & .9500 & .6277 & .0607 & 0011 \\
\hline & & 5 & - & - & 1.0000 & .8500 & .5577 & .0414 & 0007 \\
\hline & & 10 & - & - & 1.0000 & .9500 & .5340 & .0373 & .0006 \\
\hline \multirow[t]{3}{*}{5} & 5 & 2 & - & - & - & .9500 & .4204 & .0207 & .0003 \\
\hline & & 5 & - & - & - & .9500 & .3708 & .0159 & .0002 \\
\hline & & 10 & - & - & - & .9500 & 3556 & 0148 & 0002 \\
\hline \multirow[t]{3}{*}{10} & 10 & 2 & - & - & - & .9500 & .0028 & .0000 & 0000 \\
\hline & & 5 & - & - & - & .8500 & .0022 & .0000 & 0000 \\
\hline & & 10 & - & - & - & .9500 & .0022 & .0000 & 0000 \\
\hline
\end{tabular}


TABELA A.12. Continuação par* $\lambda=5$.

\begin{tabular}{|c|c|c|c|c|c|c|c|c|c|}
\hline \multicolumn{3}{|c|}{$\lambda=5$} & \multicolumn{7}{|c|}{$\rho$} \\
\hline$r$ & $s$ & $\mathrm{~K}$ & -.2000 & -.1000 & -.0500 & .0000 & .1000 & .5000 & .9000 \\
\hline \multirow[t]{3}{*}{2} & 3 & 3 & - & .7815 & .5979 & .4570 & .2827 & .0813 & .0407 \\
\hline & & 5 & - & .7671 & .5645 & .4194 & .2525 & .0752 & .0405 \\
\hline & & 10 & - & .7563 & .5428 & 3967 & .2358 & .0721 & .0403 \\
\hline \multirow[t]{3}{*}{2} & 5 & 3 & - & .9993 & .7105 & .4267 & .2037 & .0603 & .0387 \\
\hline & & 5 & - & 9998 & .6964 & .4040 & 1900 & .0584 & .0387 \\
\hline & & 10 & - & .9999 & .6858 & 3894 & 1818 & .0573 & .0387 \\
\hline \multirow[t]{3}{*}{5} & 3 & 2 & - & - & .9934 & .7026 & .2168 & .0114 & .0025 \\
\hline & & 5 & - & - & .9979 & .0331 & .1508 & .0090 & .0028 \\
\hline & & 10 & - & - & .9983 & .6060 & .1357 & .0086 & .0028 \\
\hline \multirow[t]{3}{*}{5} & 5 & $\hat{\mathrm{z}}$ & - & - & $\overline{-}$ & .6670 & .0975 & .0060 & .0024 \\
\hline & & 5 & - & - & - & .0175 & .0752 & .0056 & .0026 \\
\hline & & 10 & - & - & - & .5998 & .0702 & .0055 & .0027 \\
\hline \multirow[t]{3}{*}{10} & 10 & 2 & - & - & - & .7358 & .0000 & .0000 & .0000 \\
\hline & & 5 & - & - & - & .7153 & 0005 & 0000 & .0000 \\
\hline & & 10 & - & - & - & .7176 & 0005 & 0000 & .0000 \\
\hline
\end{tabular}

TABELA A.12. Continuação para $\lambda=20$.

\begin{tabular}{|c|c|c|c|c|c|c|c|c|c|}
\hline \multicolumn{3}{|c|}{$\lambda=5$} & \multicolumn{7}{|c|}{$\rho$} \\
\hline$r$ & $s$ & K & -.2000 & -.1000 & -.0500 & .0000 & .1000 & .5000 & .9000 \\
\hline \multirow[t]{3}{*}{ ट } & 3 & 3 & $=$ & .1371 & .0431 & .0171 & .0045 & .0004 & .0001 \\
\hline & & 5 & - & 0933 & .0271 & .0109 & .0032 & .0004 & .0002 \\
\hline & & 10 & - & .0719 & .0206 & .0086 & .0028 & .0005 & .0003 \\
\hline \multirow[t]{3}{*}{2} & 5 & 3 & - & 9469 & .0696 & .0119 & .0021 & .0003 & .002 \\
\hline & & 5 & - & .9609 & .0528 & .0092 & .0019 & .0004 & .0002 \\
\hline & & 10 & - & .9692 & .0442 & .0080 & .0018 & .0004 & .0003 \\
\hline \multirow[t]{3}{*}{5} & 3 & 2 & - & - & .8581 & .1189 & .0049 & .0001 & .0000 \\
\hline & & 5 & - & - & .8454 & .0517 & .0020 & .0001 & .0000 \\
\hline & & 10 & - & - & .8313 & .0404 & .0017 & .0001 & .0000 \\
\hline \multirow[t]{3}{*}{5} & 6 & 2 & - & - & - & .0787 & .0010 & .0000 & .0000 \\
\hline & & 5 & - & - & - & .0442 & .0007 & .0001 & .0000 \\
\hline & & 10 & - & - & - & .0378 & .0007 & .0001 & .0000 \\
\hline \multirow[t]{3}{*}{10} & 10 & 2 & - & - & - & .1237 & .0000 & .0000 & .0000 \\
\hline & & 5 & - & - & - & .0989 & .0000 & .0000 & .0000 \\
\hline & & 10 & - & - & - & .0976 & .0000 & .0000 & .0000 \\
\hline
\end{tabular}


TABELA A.13. Probabilidades do erro tipo I, para Ho? Ho $\mathrm{H}^{(2)}$, Ho ao nivel nominal de significancia 0,05 segundo os valores de $r, s, k, \rho_{1}, \rho_{2}=0.1 ; 0.3$ e. 0.5 .

\begin{tabular}{|c|c|c|c|c|c|c|c|c|c|c|}
\hline & $P_{2}$ & $=$ & 0.4 & & & & $P_{i}$ & & & \\
\hline$r$ & Is & $k$ & $\mathrm{H}^{*}$ & -.9000 & -.5000 & -.1000 & .0000 & .1000 & .5000 & .9000 \\
\hline$\overline{2}$ & 3 & 3 & 1 & - & - & .0773 & .1105 & .1492 & .3580 & .7151 \\
\hline & & & 2 & - & - & .0049 & .0196 & .0493 & .3500 & .8576 \\
\hline & & & 3 & - & - & .0049 & .0196 & .0499 & .3509 & .8576 \\
\hline & & 5 & 1 & - & - & .1007 & $\because 1583$ & .2194 & 4736 & .7856 \\
\hline & & & $a$ & - & - & .0000 & .0046 & .0505 & .5171 & .9209 \\
\hline & & & 3 & - & - & .0000 & .0046 & .0505 & .5171 & .9209 \\
\hline & & 10 & 1 & - & - & - & - & .3409 & 6077 & .8502 \\
\hline & & & $a$ & - & - & - & - & .0508 & .7063 & .0610 \\
\hline & & & 3 & - & - & - & - & .0508 & .7063 & .9609 \\
\hline$\overline{2}$ & 5 & 3 & 1 & - & - & 1376 & .1750 & .2162 & .4194 & .7449 \\
\hline & & & 2 & - & - & .0010 & .0138 & .0498 & .5137 & .9758 \\
\hline & & & 3 & - & - & .0019 & .0138 & .0498 & .5137 & .9758 \\
\hline & & 5 & 1 & - & - & .1964 & .2504 & 3049 & .5266 & .8049 \\
\hline & & & 2 & - & - & .0000 & .0018 & .0497 & .7201 & .9022 \\
\hline & & & 3 & - & - & .0000 & .0018 & .0497 & 7201 & .9922 \\
\hline & & 10 & 1 & - & - & - & - & .4414 & .6508 & .8831 \\
\hline & & & 2 & - & - & - & - & .0518 & .8952 & 9982 \\
\hline & & & 3 & - & - & - & - & .0518 & .8952 & .9982 \\
\hline 5 & 3 & 2 & 1 & $=$ & .0085 & .0782 & .1201 & .1745 & .5407 & .9678 \\
\hline & & & a & - & .0001 & .0137 & .0277 & .0501 & .2735 & .8078 \\
\hline & & & 3 & - & .0000 & .0086 & .0222 & .0501 & .4650 & .8940 \\
\hline & & 5 & 1 & - & - & .1532 & .2938 & .4423 & .8661 & .9960 \\
\hline & & & 2 & - & - & .0000 & .0033 & .0500 & .5361 & .8262 \\
\hline & & & 3 & - & - & .0000 & .0005 & .0497 & .9046 & .9999 \\
\hline & & 10 & 1 & - & - & - & - & .6968 & .9588 & 9998 \\
\hline & & & 2 & - & - & - & - & .0521 & .7155 & .9634 \\
\hline & & & 3 & - & - & - & - & .0561 & .9873 & 1.0000 \\
\hline 5 & 5 & 2 & 1 & - & .0303 & .1632 & .2230 & .2944 & .6661 & .9807 \\
\hline & & & 2 & - & .0000 & .0084 & .0224 & .0499 & 3962 & .9554 \\
\hline & & & 3 & - & .0000 & .0038 & .0153 & .0477 & .6696 & 1.0000 \\
\hline & & 5 & 1 & - & - & . 3923 & .5175 & .6292 & 9121 & .9973 \\
\hline & & & 2 & - & - & .0000 & .0012 & .0510 & 7431 & .9933 \\
\hline & & & 3 & - & - & .0000 & .0001 & .0492 & .9889 & 1.0000 \\
\hline & & 10 & 1 & - & - & - & - & .8395 & .9735 & .9994 \\
\hline & & & 2 & - & - & - & - & .0534 & .8013 & .8983 \\
\hline & & & 3 & - & - & - & - & .0537 & .9998 & 1.0000 \\
\hline 10 & 10 & 2 & 1 & - & .2877 & .6480 & .7329 & .8080 & .9775 & 1.0000 \\
\hline & & & 2 & - & .0000 & .0037 & .0189 & .0635 & .6867 & .9084 \\
\hline & & & 3 & - & .0000 & .0010 & .0225 & .1716 & .9997 & 1.0000 \\
\hline & & 5 & 1 & - & - & .9321 & .9606 & .9782 & .9991 & 1.0000 \\
\hline & & & 2 & - & - & .0000 & .0001 & .0534 & .9463 & 1.0000 \\
\hline & & & 3 & - & - & .0000 & .0000 & .1120 & 1.0000 & 1.0000 \\
\hline & & 10 & 1 & - & - & - & - & .9978 & .9909 & 1.0000 \\
\hline & & & 2 & - & - & - & - & .0515 & .9032 & 1.0000 \\
\hline & & & 3 & - & - & - & - & .0984 & 1.0000 & 1. 0000 \\
\hline
\end{tabular}


TABELA A.13. Contiruação para $P_{2}=0.3$ segundo

\begin{tabular}{|c|c|c|c|c|c|c|c|c|c|c|}
\hline & $P_{2}$ & $=0$ & 0.3 & & & & $P_{1}$ & & & \\
\hline$r$ & ss & $k$ & $\mathrm{H}^{2}$ & -.0500 & 0000 & .1000 & .2000 & .5000 & .7000 & .9000 \\
\hline$\overline{2}$ & 13 & 3 & 1 & - & .2172 & .2556 & .2972 & .4445 & .5710 & .7534 \\
\hline & & & 2 & - & .0000 & .0015 & .0140 & 2123 & .4812 & .8177 \\
\hline & & & 3 & - & .0000 & 0015 & .0140 & .2123 & .4812 & .8177 \\
\hline & & 5 & 1 & - & - & - & .4788 & .5578 & .6692 & .8153 \\
\hline & & & 2 & - & - & - & .0012 & .3396 & .6478 & 8968 \\
\hline & & & 3 & - & - & - & .0012 & . 3396 & .6478 & .8968 \\
\hline & & 10 & 1 & - & - & - & - & 8346 & .8740 & .9286 \\
\hline & & & 2 & - & - & - & - & .5365 & .8034 & 9498 \\
\hline & & & 13 & - & - & - & - & .5365 & .8034 & 9498 \\
\hline$\overline{2}$ & 5 & 3 & 11 & - & .3424 & .3772 & .4140 & .5402 & .6460 & .6979 \\
\hline & & & 2 & - & .0000 & .0003 & .0086 & .3021 & 6847 & 9603 \\
\hline & & & 3 & - & .0000 & .0003 & .0086 & .3021 & 6847 & 9603 \\
\hline & & 5 & 1 & - & - & - & .5214 & .6366 & .7256 & 8454 \\
\hline & & & 2 & - & - & - & .0002 & .4903 & 8491 & 9868 \\
\hline & & & 3 & - & - & - & .0002 & 4903 & .8491 & 9868 \\
\hline & & 10 & 1 & - & - & - & - & 7387 & .8056 & 8918 \\
\hline & & & 2 & - & - & - & - & 7431 & .9527 & 9969 \\
\hline & & & 3 & - & - & - & - & .7431 & .9527 & 9909 \\
\hline 5 & 13 & 2 & 1 & .2535 & 2843 & 3523 & .4285 & .6907 & .8624 & 9812 \\
\hline & & & 2 & 0011 & 0025 & .0087 & .0229 & .1640 & 3899 & 7574 \\
\hline & & & 3 & 0003 & .0009 & .0046 & .0171 & .2487 & .6721 & .9857 \\
\hline & & 5 & 1 & - & - & - & .7924 & .9315 & .9780 & .9978 \\
\hline & & & 2 & - & - & - & .0006 & .3563 & 6649 & .9035 \\
\hline & & & 3 & - & - & - & .0000 & .6902 & 9721 & 9998 \\
\hline & & 10 & 1 & - & - & - & - & 9808 & 9945 & 9995 \\
\hline & & & 2 & - & - & - & - & 5481 & .8103 & .9517 \\
\hline & & & 3 & - & - & - & - & .9233 & .9974 & 1.0000 \\
\hline 5 & 5 & 2 & 1 & .4984 & .5295 & .5930 & .6570 & .8389 & .9349 & .9919 \\
\hline & & & 2 & .0002 & .0007 & .0044 & .0173 & .2230 & 5657 & .9291 \\
\hline & & & 3 & 0000 & 0001 & 0018 & .0118 & 3751 & .8851 & .9998 \\
\hline & & 5 & 1 & - & - & - & .9083 & .9680 & .9897 & .9989 \\
\hline & & & 2 & - & - & - & .0001 & .5175 & .8643 & .9886 \\
\hline & & & 3 & - & - & - & .0000 & .8898 & .9992 & 1.0000 \\
\hline & & 10 & 1 & - & - & - & - & 9916 & .9974 & .9998 \\
\hline & & & $a$ & - & - & - & - & 7548 & .9558 & .9971 \\
\hline & & & 3 & - & - & - & - & .9931 & 1.0000 & 1.0000 \\
\hline 10 & 10 & 2 & 1 & .9698 & .9750 & .9836 & .9898 & .9986 & 9998 & 1.0000 \\
\hline & & & 2 & .0000 & .0000 & .0012 & .0125 & 4107 & .8523 & .9982 \\
\hline & & & 3 & .0000 & .0000 & .0001 & .0104 & 9648 & 1.0000 & 1.0000 \\
\hline & & 5 & 1 & - & - & - & .9996 & 1.0000 & 1.0000 & 1.0000 \\
\hline & & & 2 & - & - & - & .0000 & .7744 & .9875 & 1.0000 \\
\hline & & & 3 & - & - & - & .0000 & 1.0000 & 1.0000 & 1.0000 \\
\hline & & 10 & 1 & - & - & - & - & 1.0000 & 1.0000 & 1.0000 \\
\hline & & & $\mathrm{e}$ & - & - & - & - & .9468 & 9969 & 1.0000 \\
\hline & & & 3 & - & - & - & - & 1.0000 & 1.0000 & 1.0000 \\
\hline
\end{tabular}


TABELA A.13. Contiruação para $\rho_{2}=0.5$ segundo $\rho_{1}$

\begin{tabular}{|c|c|c|c|c|c|c|c|c|c|c|}
\hline & $P_{2}$ & $=$ & 0.5 & & & & $\rho_{i}$ & & & \\
\hline & Is & $K$ & $H^{*}$ & 0000 & 1000 & 3000 & .4000 & .5000 & .6000 & .9000 \\
\hline & 3 & 3 & 11 & - & - & .4119 & .4559 & .5038 & .5568 & .7796 \\
\hline & & & 2 & - & - & .0000 & .0068 & .0499 & 1509 & 7468 \\
\hline & & & 3 & - & - & 0000 & 0068 & .0499 & 1509 & .7468 \\
\hline & & 5 & 1 & - & - & - & .5683 & .6114 & 6572 & .8353 \\
\hline & & & 2 & - & - & - & .0000 & .0505 & .2419 & .8519 \\
\hline & & & 3 & - & - & - & .0000 & .0505 & .2419 & .8519 \\
\hline & & 10 & 1 & - & - & - & - & .7209 & .7509 & 8856 \\
\hline & & & 2 & - & - & - & - & .0505 & .4126 & .9202 \\
\hline & & & 3 & - & - & - & - & .0505 & 4126 & .9202 \\
\hline$\overline{2}$ & 5 & 3 & 1 & - & - & 5339 & .5694 & .6078 & 6500 & .8264 \\
\hline & & & 2 & - & - & .00 & .00 & .04 & 2034 & 9237 \\
\hline & & & 3 & - & - & .0000 & .0030 & .0498 & 2034 & .9237 \\
\hline & & 5 & 1 & - & - & - & 6617 & 68 & 7289 & .8682 \\
\hline & & & 12 & - & - & - & .00 & .0 & 29 & 9729 \\
\hline & & & 3 & - & - & - & .0000 & .04 & 29 & 9729 \\
\hline & & 10 & 1 & - & - & - & - & $.7 \varepsilon$ & & 9078 \\
\hline & & & e & - & - & - & - & .05 & 40 & 9933 \\
\hline & & & 3 & - & - & - & - & .0518 & 5940 & .9933 \\
\hline 5 & 3 & 2 & 1 & - & .593 & .6746 & .7171 & .7610 & 8062 & .4496 \\
\hline & & & le & - & .0 & .00 & .00 & .0 & 10 & 7905 \\
\hline & & & 3 & - & .0000 & .0013 & 01 & .0 & 1644 & 9574 \\
\hline & & 5 & 1 & - & - & - & .8867 & .96 & 9274 & 9820 \\
\hline & & & 12 & - & - & - & .00 &. $\mathrm{O}^{-}$ & 19 & .9623 \\
\hline & & & 3 & - & - & - & 0000 & .0 & 2 & 9 \\
\hline & & 10 & 1 & - & - & - & - & 9 & 43 & 9 \\
\hline & & & 2 & - & - & - & - & .0 & $4 \varepsilon$ & 9 \\
\hline & & & 3 & - & - & - & - & .0 & 30 & .9990 \\
\hline 5 & 5 & 2 & 1 & - & .7451 & .8315 & 8707 & .80 & .9376 & .9956 \\
\hline & & & 2 & - & .00 & .0010 & 01 & .0 & & $8 ?$ \\
\hline & & & 3 & - & .0000 & .0002 & .0052 & .0 & 24 & .95 \\
\hline & & 5 & 1 & - & - & - & .9783 & .98 & .9901 & 9994 \\
\hline & & & 2 & - & - & - & .0000 & .0510 & 3662 & .9763 \\
\hline & & & 3 & - & - & - & .0000 & .0 & .7067 & 1.0000 \\
\hline & & 10 & 1 & - & - & - & - & .9959 & 9975 & 9999 \\
\hline & & & 12 & - & - & - & - & .0534 & 0085 & 9938 \\
\hline & & & 3 & - & - & - & - & .05 & 9563 & 1.0000 \\
\hline 10 & 10 & 2 & 1 & - & .9968 & .9989 & .9994 & 8997 & 9990 & 1.0000 \\
\hline & & & 2 & - & .0000 & .0001 & .0054 & .0635 & 2719 & ๑९حE \\
\hline & & & 3 & - & .0000 & 0000 & .0021 & .1716 & .8322 & 1.0000 \\
\hline & & 5 & 1 & - & - & - & 1.0000 & 1.0000 & 1.0000 & 1.0000 \\
\hline & & & 2 & - & - & - & .0000 & .0534 & .5836 & .9998 \\
\hline & & & 3 & - & - & - & .0000 & .1120 & .9095 & 1.0000 \\
\hline & & 10 & 1 & - & - & - & - & 1.0000 & 1.0000 & 1.0000 \\
\hline & & & 2 & - & - & - & - & .0515 & .8481 & 1.0000 \\
\hline & & & 3 & - & - & - & - & .0984 & 1.0000 & 1.0000 \\
\hline
\end{tabular}


TABELA A.14. Probabilidades do erro tipo II, para $H_{0}^{(1)} H_{0}^{(2)}$ Ho: a nivel nominal de significancia 0.05 . segundo os vallores de $r, s, k, \rho_{1}, \rho_{2}=0.3 e \lambda$.

\begin{tabular}{|c|c|c|c|c|c|c|c|c|c|c|}
\hline & $=0$ & $\rho_{2}=$ & 3.3 & & & & $P_{i}$ & & & \\
\hline$r$ & 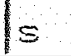 & $k$ & $\mathrm{H}^{*}$ & $1-.0500$ & .0000 & .1000 & .2000 & .5000 & .7000 & .9000 \\
\hline $\bar{z}$ & 3 & 3 & 1 & - & 7825 & .7444 & .7028 & .5555 & .4200 & .2466 \\
\hline & & & 2 & - & 1.0000 & .9085 & 9860 & .7877 & .5188 & .1823 \\
\hline & & & 3 & - & 1.0000 & .9985 & .9860 & .7877 & .5188 & .1823 \\
\hline & & 5 & 1 & - & - & - & .5912 & .4422 & .3308 & .1847 \\
\hline & & & 2 & - & - & - & 9988 & .6604 & 3522 & .1032 \\
\hline & & & 3 & - & - & - & 9988 & .6604 & .3522 & .1032 \\
\hline & & 10 & 1 & - & - & - & - & .1654 & .1260 & .0714 \\
\hline & & & 2 & - & - & - & - & .4535 & 1966 & .0502 \\
\hline & & & 3 & - & - & - & - & .4835 & 1966 & .0502 \\
\hline$\overline{2}$ & 5 & 3 & 1 & - & .6576 & .6228 & .5860 & .4598 & 3540 & .2031 \\
\hline & & & 2 & - & 1.0000 & 9997 & .9914 & 6979 & 3153 & .0397 \\
\hline & & & 3 & - & 1.0000 & 9997 & 9914 & 6979 & .3153 & .0397 \\
\hline & & 5 & 1 & - & - & - & .4786 & .3634 & .2744 & .1546 \\
\hline & & & 2 & - & - & - & 9998 & .5096 & .1509 & .0132 \\
\hline & & & 3 & - & - & - & .9998 & .5006 & .1509 & .0132 \\
\hline & & 10 & 1 & - & - & - & - & .2613 & .1044 & .1082 \\
\hline & & & 2 & - & - & - & - & .2569 & .0473 & .0031 \\
\hline & & & 3 & - & - & - & - & 2569 & .0473 & .0031 \\
\hline 5 & 3 & 2 & 1 & .7465 & 7157 & 6477 & .5715 & .3083 & .1376 & .0188 \\
\hline & & & 2 & 9989 & 9975 & 9913 & .9771 & .8360 & 6101 & .2426 \\
\hline & & & 3 & 9997 & 9991 & 9954 & 9829 & .7513 & 3279 & .0143 \\
\hline & & 5 & 1 & - & - & - & 2076 & .0685 & .0220 & .0022 \\
\hline & & & 12 & - & - & - & .9994 & .6437 & .3351 & .0965 \\
\hline & & & 3 & - & - & - & 1.0000 & 3098 & .0279 & .0002 \\
\hline & & 10 & 1 & - & - & - & - & .0192 & .0055 & .0005 \\
\hline & & & 2 & - & - & - & - & .4519 & .1897 & .0483 \\
\hline & & & 3 & - & - & - & - & .0767 & $.002 E$ & .0000 \\
\hline 5 & 5 & 2 & 11 & 5016 & 4705 & 4070 & .3430 & .1611 & .0651 & .0081 \\
\hline & & & 2 & 9998 & 9993 & 9956 & .9827 & .7770 & .4343 & .0709 \\
\hline & & & 3 & 1.0000 & 9999 & 9982 & 9982 & 6249 & 1149 & .0002 \\
\hline & & 5 & 1 & - & - & - & .0917 & .0314 & .0103 & .0011 \\
\hline & & & 2 & - & - & - & 9999 & .4825 & .1357 & .0114 \\
\hline & & & 13 & - & - & - & 1.0000 & .1102 & .0008 & .0000 \\
\hline & & 10 & 1 & - & - & - & - & .0084 & .0026 & .0002 \\
\hline & & & 2 & - & - & - & - & .2452 & .0442 & .0020 \\
\hline. & & & 3 & - & - & - & - & .0059 & .0000 & .0000 \\
\hline 10 & 10 & 2 & 1 & .0302 & .0250 & 0164 & .0102 & .0014 & .0002 & .0000 \\
\hline & & & 2 & 1.0000 & 1.0000 & 9988 & .9875 & .5893 & .1377 & .0018 \\
\hline & & & 3 & 1.0000 & 1.0000 & 9999 & 9896 & .0352 & .0000 & .0000 \\
\hline & & 5 & 1 & - & - & - & .0004 & 0000 & .0000 & .0000 \\
\hline & & & 2 & - & - & - & 1.0000 & .2256 & .0125 & 0000 \\
\hline & & & 3 & - & - & - & 1.0000 & .0000 & .0000 & .0000 \\
\hline & & 10 & 1 & - & - & - & - & .0000 & .0000 & .0000 \\
\hline & & & 2 & - & - & - & - & .0532 & .0011 & .0000 \\
\hline & & & 3 & - & - & - & - & .0000 & 0000 & .0000 \\
\hline
\end{tabular}


TABELAS A.14, Continuaga para $\lambda=5$.

\begin{tabular}{|c|c|c|c|c|c|c|c|c|c|c|}
\hline$\lambda=$ & $=5$ & $\rho_{2}=$ & 3.2 & & & & $\rho_{1}$ & & & \\
\hline$r$ & Is & $\mathrm{K}$ & $\mathrm{H}^{*}$ & $1-.0500$ & .0000 & .1000 & .2000 & 5000 & .7000 & .8000 \\
\hline$\overline{2}$ & 13 & 3 & 1 & - & 1705 & .1452 & .1237 & .0772 & .0567 & 0417 \\
\hline & & & 2 & - & 9995 & 9538 & .7973 & .2666 & 0991 & 0301 \\
\hline & & & 3 & - & 9995 & .9538 & .7973 & 2660 & 0991 & 0301 \\
\hline & & 5 & 1 & - & - & - & .0826 & 0574 & .0459 & 0302 \\
\hline & & & 2 & - & - & - & .9385 & 1519 & .0537 & $022 ?$ \\
\hline & & & 3 & - & - & - & .9385 & 1519 & .0537 & .0227 \\
\hline & & 10 & 1 & - & - & - & - & .0397 & .0377 & .0368 \\
\hline & & & 2 & - & - & - & - & .0756 & .0316 & .0190 \\
\hline & & & 3 & - & - & - & - & .0756 & .0316 & .0190 \\
\hline $\bar{z}$ & 5 & 3 & 1 & - & .1018 & .0913 & .0820 & .0598 & .0487 & .0390 \\
\hline & & & 2 & - & 1.0000 & .9894 & .8901 & .2635 & .0342 & .0088 \\
\hline & & & 3 & - & 1.0000 & .9894 & 9801 & .2635 & .0642 & $.008 \mathrm{~B}$ \\
\hline & & 5 & 1 & - & - & - & .0616 & .0493 & .0432 & .0384 \\
\hline & & & 2 & - & - & - & 9861 & 1306 & .0255 & .0051 \\
\hline & & & 3 & - & - & - & .9861 & 1308 & .0255 & 0051 \\
\hline & & 10 & 1 & - & - & - & - & 0425 & .0397 & .0374 \\
\hline & & & e & - & - & - & - & 0458 & .0099 & 0035 \\
\hline & & & 3 & - & - & - & - & .0458 & .0099 & .0035 \\
\hline 5 & 13 & 2 & 1 & 3231 & 2913 & . 2319 & .1789 & .0542 & .0238 & .0055 \\
\hline & & & 2 & 9556 & 9242 & .8325 & 7107 & 3158 & .1330 & .0373 \\
\hline & & & 3 & .9967 & 9922 & .8690 & 9121 & 4487 & .1081 & .0027 \\
\hline & & 5 & 1 & - & - & - & .0358 & .0127 & .0064 & 0032 \\
\hline & & & 2 & - & - & - & .9425 & .2368 & .0488 & .0224 \\
\hline & & & 3 & - & - & - & 9986 & .0856 & .0051 & .0002 \\
\hline & & 10 & 1 & - & - & - & - & .0061 & .0040 & .0028 \\
\hline & & & 2 & - & - & - & - & .0719 & .0309 & .0191 \\
\hline & & & 3 & - & - & - & - & .0144 & .0007 & .0001 \\
\hline 5 & 5 & 2 & 1 & .1316 & 1176 & .0925 & .0712 & .0276 & .0121 & .0042 \\
\hline & & & 2 & .9907 & 9780 & 9222 & 8165 & .3355 & .1028 & .0130 \\
\hline & & & 3 & .9997 & 9990 & 9907 & 9534 & .3873 & .0374 & .0000 \\
\hline & & 5 & 1 & - & - & - & 0160 & .078 & .0048 & .0030 \\
\hline & & & 2 & - & - & - & 9883 & .1143 & .0227 & .0050 \\
\hline & & & 3 & - & - & - & 1.0000 & .0311 & .0002 & .0000 \\
\hline & & 10 & 1 & - & - & - & - & .0046 & .0035 & .0027 \\
\hline & & & 2 & - & - & - & - & .0428 & .0095 & .0035 \\
\hline & & & 3 & - & - & - & - & .0013 & .0000 & .0000 \\
\hline 10 & 10 & 2 & 1 & .0055 & 0045 & .0030 & .0019 & .0004 & .0001 & .0000 \\
\hline & & & 2 & 9997 & 9982 & 9766 & .8882 & .2465 & .0303 & .0005 \\
\hline & & & 3 & 1.0000 & 1.0000 & 9997 & .9788 & .0185 & .0000 & .0000 \\
\hline & & 5 & 1 & - & - & - & .0002 & .0000 & .0000 & .0000 \\
\hline & & & 2 & - & - & - & .9996 & .0557 & .0023 & .0001 \\
\hline & & & 3 & - & - & - & 1.0000 & .0000 & .0000 & .0000 \\
\hline & & 10 & 1 & - & - & - & - & .000 & .0000 & .0000 \\
\hline & & & 2 & - & - & - & - & .0098 & .0003 & .0000 \\
\hline & & & 3 & - & - & - & - & .0000 & .0000 & .0000 \\
\hline
\end{tabular}


IABELA A.14. Continuaçz̃o para $\lambda=20$.

\begin{tabular}{|c|c|c|c|c|c|c|c|c|c|c|}
\hline$\overline{\lambda=}$ & 201 & $\rho_{2}=$ & 5.3 & & & & $P_{1}$ & & & \\
\hline$r$ & Is & $K$ & $\mathrm{H}^{*}$ & -.0500 & .0000 & .1000 & .2000 & .5000 & .7000 & .9000 \\
\hline$\overline{2}$ & 13 & 3 & 11 & - & .0014 & .0010 & .0003 & .0003 & $.000 \hat{~}$ & .0001 \\
\hline & & & $\sqrt{2}$ & - & 9810 & .5371 & .1761 & .0052 & .0007 & .0001 \\
\hline & & & 3 & - & 9810 & .5371 & .1761 & .0052 & .0007 & .0001 \\
\hline & & 5 & 1 & - & - & - & .0005 & .0003 & .0002 & .0002 \\
\hline & & & 2 & - & - & - & .3710 & .0015 & .0003 & .0001 \\
\hline & & & 3 & - & - & - & .3710 & .0015 & .0003 & .0001 \\
\hline & & 10 & 1 & - & - & - & - & .0003 & .0003 & .0003 \\
\hline & & & e & - & - & - & - & .0006 & .0002 & .0001 \\
\hline & & & 3 & - & - & - & - &. $\mathrm{Or}$ & .0002 & .0001 \\
\hline $\bar{z}$ & 15 & 3 & 1 & - & 0006 & 0005 & 0004 & .0003 & .0002 & .0002 \\
\hline & & & 12 & - & 9992 & .77 & .3127 &.$O C$ & .0005 & .0000 \\
\hline & & & 3 & - & 9992 & .7706 & .3127 & .0068 & .0005 & .0000 \\
\hline & & 5 & 1 & - & - & - & .0004 & .0003 & .0002 & .0002 \\
\hline & & & 12 & - & - & - & .6518 & .0016 & .0002 & .0000 \\
\hline & & & 3 & - & - & - & .6518 & .0016 & .0002 & .0000 \\
\hline & & 10 & 1 & - & - & - & - & .0003 & .0003 & .0003 \\
\hline & & & 2 & - & - & - & - & .00 &.$O S$ & .0000 \\
\hline & & & 3 & - & - & - & - & .00 & .0 & .0000 \\
\hline 5 & 3 & iz & 1 & .013 & 0094 & .0055 & .0033 &. $\mathrm{OC}$ & .0 & .0000 \\
\hline & & & 2 & $.50^{\circ}$ & 38 & 19 & .09 & .0 &.$O$ & $0 己$ \\
\hline & & & 3 & .9398 & 8869 & 7191 & .4992 & .04 & .0026 & .0000 \\
\hline & & 15 & 1 & - & - & - & .0003 & .00 &.$O C$ & .0000 \\
\hline & & & 2 & - & - & - & .3156 &. $\mathrm{O}$ & .0 & .0002 \\
\hline & & & 3 & - & - & - & .9048 & .0 & .0 & .0000 \\
\hline & & 10 & 1 & - & - & - & - & .0 & .0 & .0000 \\
\hline & & & 2 & - & - & - & - & .0 & .0 & .0002 \\
\hline & & & 3 & - & - & - & - &.$O C$ &.$O C$ & .0000 \\
\hline 5 & 5 & $\hat{E}$ & 1 & 0017 & 0014 & .0009 & .0006 & .0002 & .0001 & .0000 \\
\hline & & & a & 760 & 6273 & 366 & .1812 & .01 &.$O C$ & .0001 \\
\hline & & & 3 & 9922 & 9767 & 8854 & .5821 & .05 & .0010 & .0000 \\
\hline & & 5 & 1 & - & - & - & .0001 &.$O C$ & .0001 & .0000 \\
\hline & & & 2 & - & - & - & .6144 & .00 & .0 & .0001 \\
\hline & & & 3 & - & - & - & .9934 &.$O C$ & .0 & .0 \\
\hline & & 10 & 1 & - & - & - & - &.$O$ & .0 & .0 \\
\hline & & & 2 & - & - & - & - &.$O C$ & & \\
\hline & & & 3 & - & - & - & - & .00 &.$O C$ & .0 \\
\hline 10 & 10 & $\overline{2}$ & 1 & .0000 & 0000 & .0000 & .0000 & .0000 & .0000 & .0000 \\
\hline & & & 2 & 8560 & 8742 & .5813 & 2825 & .00 & .00 & .0000 \\
\hline & & & 3 & 1.0000 & 1.0000 & 9960 & 8902 & 0022 & .00 & .0000 \\
\hline & & 5 & 1 & - & - & - & .0000 & 0000 & .00 & .0000 \\
\hline & & & 2 & - & - & - & 9292 & & 0 & .0 \\
\hline & & & 3 & - & - & - & 1.0000 & 00 & .0000 & .0000 \\
\hline & & 10 & 1 & - & - & - & - & 0000 & .0000 & .0000 \\
\hline & & & 2 & - & - & - & - & 00 & .00 & .0000 \\
\hline & & & $\Xi$ & - & - & - & - & 0000 & .0000 & .0000 \\
\hline
\end{tabular}

